\title{
Basement membrane stiffness determines metastases formation
}

\author{
Raphael Reuten ${ }^{1 凶}$, Sina Zendehroud ${ }^{20,17}$, Monica Nicolau ${ }^{1,17}$, Lutz Fleischhauer ${ }^{3,4}$, Anu Laitala', \\ Stefanie Kiderlen ${ }^{3}{ }^{3,4}$, Denise Nikodemus' ${ }^{1}$, Lena Wullkopf1, Sebastian Rune Nielsen (1)', \\ Sarah McNeilly5 , Carina Prein ${ }^{3,4,16}$, Maria Rafaeva1', Erwin M. Schoof ${ }^{1,6,7,8}$, Benjamin Furtwängler (1),7,8, \\ Bo T. Porse ${ }^{1,7,8}$, Hyobin Kim ${ }^{1,8}{ }^{1,8}$ Kyoung Jae Won ${ }^{1,8}$, Stefanie Sudhop ${ }^{4}$, Kamilla Westarp Zornhagen ${ }^{1}$, \\ Frank Suhr ${ }^{9}$, Eleni Maniati ${ }^{10}$, Oliver M. T. Pearce ${ }^{10}$, Manuel Koch ${ }^{11,12}$, Lene Broeng Oddershede ${ }^{13}$, \\ Tom Van Agtmael ${ }^{5}$, Chris D. Madsen ${ }^{14}$, Alejandro E. Mayorca-Guiliani', Wilhelm Bloch ${ }^{15}$, \\ Roland R. Netz $\mathbb{B}^{2}$, Hauke Clausen-Schaumann $\mathbb{B}^{3,4}$ and Janine T. Erler ${ }^{1}{ }^{1 凶}$
}

\begin{abstract}
The basement membrane (BM) is a special type of extracellular matrix and presents the major barrier cancer cells have to overcome multiple times to form metastases. Here we show that BM stiffness is a major determinant of metastases formation in several tissues and identify netrin-4 (Net4) as a key regulator of BM stiffness. Mechanistically, our biophysical and functional analyses in combination with mathematical simulations show that Net4 softens the mechanical properties of native BMs by opening laminin node complexes, decreasing cancer cell potential to transmigrate this barrier despite creating bigger pores. Our results therefore reveal that BM stiffness is dominant over pore size, and that the mechanical properties of 'normal' BMs determine metastases formation and patient survival independent of cancer-mediated alterations. Thus, identifying individual Net4 protein levels within native BMs in major metastatic organs may have the potential to define patient survival even before tumour formation. The ratio of Net4 to laminin molecules determines BM stiffness, such that the more Net4, the softer the BM, thereby decreasing cancer cell invasion activity.
\end{abstract}

C ancer metastasis is responsible for $66-90 \%$ of patient deaths ${ }^{1,2}$. Cancer cells encounter and must breach basement membranes (BMs) several times during the metastatic process-during invasion, intravasation and extravasation ${ }^{3,4}$.

The BM, a specialized type of extracellular matrix (ECM) in direct contact with cells, is shaped by two major ECM macromolecule proteins, collagen IV and laminin, which self-assemble into two supramolecular polymers ${ }^{5}$. The collagen IV network is deposited on top of the laminin network, which is the key determinant that maintains cell-BM interaction and mechanical stability ${ }^{6}$. Each laminin chain contains a laminin N-terminal globular domain, and interactions between laminin $\mathrm{N}$-terminal domains from distinct laminin heterotrimers generate the laminin network.

The BM is a dense, nanoporous sheet with a pore size between 10 and $112 \mathrm{~nm}^{7-9}$. Currently, there are two paradigms describing the process of BM breaching. According to the first paradigm, cancer cells can form actin- and protease-rich invadosomes, which can proteolytically degrade BM components to generate an entry site to breach ${ }^{10}$. Recent studies have revealed that cells can transmigrate across the BM in a protease-independent manner. Cancer cells can form invadosomes to plastically generate channels to migrate through the $\mathrm{BM}^{11}$. According to the second paradigm, cancer-associated fibroblasts (CAFs) can pull and stretch the BM in a protease-independent manner to generate gaps via which cancer cells can pass through the $\mathrm{BM}^{12}$. However, these paradigms of $\mathrm{BM}$ breaching exclusively focus on forces applied by cells on the BM and the functional role of the BM's mechanical properties has so far not been demonstrated.

Here we provide evidence for a functional role of BM architecture during the invasion-metastasis process in vitro, in animal models and in several patient tumour types. We examine the relationship between BM composition and cancer patient survival, and identify

'Biotech Research and Innovation Centre, University of Copenhagen, Copenhagen, Denmark. ${ }^{2}$ Department of Physics, Freie Universität Berlin, Berlin, Germany. ${ }^{3}$ Center for Applied Tissue Engineering and Regenerative Medicine-CANTER, Munich University of Applied Sciences, Munich, Germany. ${ }^{4}$ Center for Nanoscience-CeNS, Munich, Germany. ${ }^{5}$ Institute of Cardiovascular and Medical Sciences, College of Medical, Veterinary and Life Sciences, University of Glasgow, Glasgow, UK. ${ }^{6}$ Department of Biotechnology and Biomedicine, Technical University of Denmark, Lyngby, Denmark. ${ }^{7}$ The Finsen Laboratory, Rigshospitalet, Faculty of Health Sciences, University of Copenhagen, Copenhagen, Denmark. ${ }^{8}$ Novo Nordisk Foundation Center for Stem Cell Biology, DanStem, Faculty of Health Sciences, University of Copenhagen, Copenhagen, Denmark. 'Exercise Physiology Research Group, Department of Movement Sciences, Biomedical Sciences Group, KU Leuven, Leuven, Belgium. ${ }^{10}$ Centre for Tumour Microenvironment, Barts Cancer Institute, Queen Mary University of London, London, UK. "Center for Biochemistry, Center for Molecular Medicine Cologne (CMMC), Faculty of Medicine and University Hospital Cologne, University of Cologne, Cologne, Germany. ${ }^{12}$ Institute for Dental Research and Oral Musculoskeletal Biology, Faculty of Medicine and University Hospital Cologne, University of Cologne, Cologne, Germany. ${ }^{13}$ Niels Bohr Institute, University of Copenhagen, Copenhagen, Denmark. ${ }^{14}$ Department of Laboratory Medicine, Division of Translational Cancer Research, Lund University, Lund, Sweden. ${ }^{15}$ Institute of Cardiovascular Research and Sport Medicine, Department of Molecular and Cellular Sport Medicine, German Sport University Cologne, Cologne, Germany. ${ }^{16}$ Present address: Department of Physiology and Pharmacology, Schulich School of Medicine \& Dentistry, University of Western Ontario, London, Ontario, Canada. ${ }^{17}$ These authors contributed equally: S. Zendehroud, M. Nicolau. $\bowtie$ e-mail: raphael.reuten@bric.ku.dk; janine.erler@bric.ku.dk 

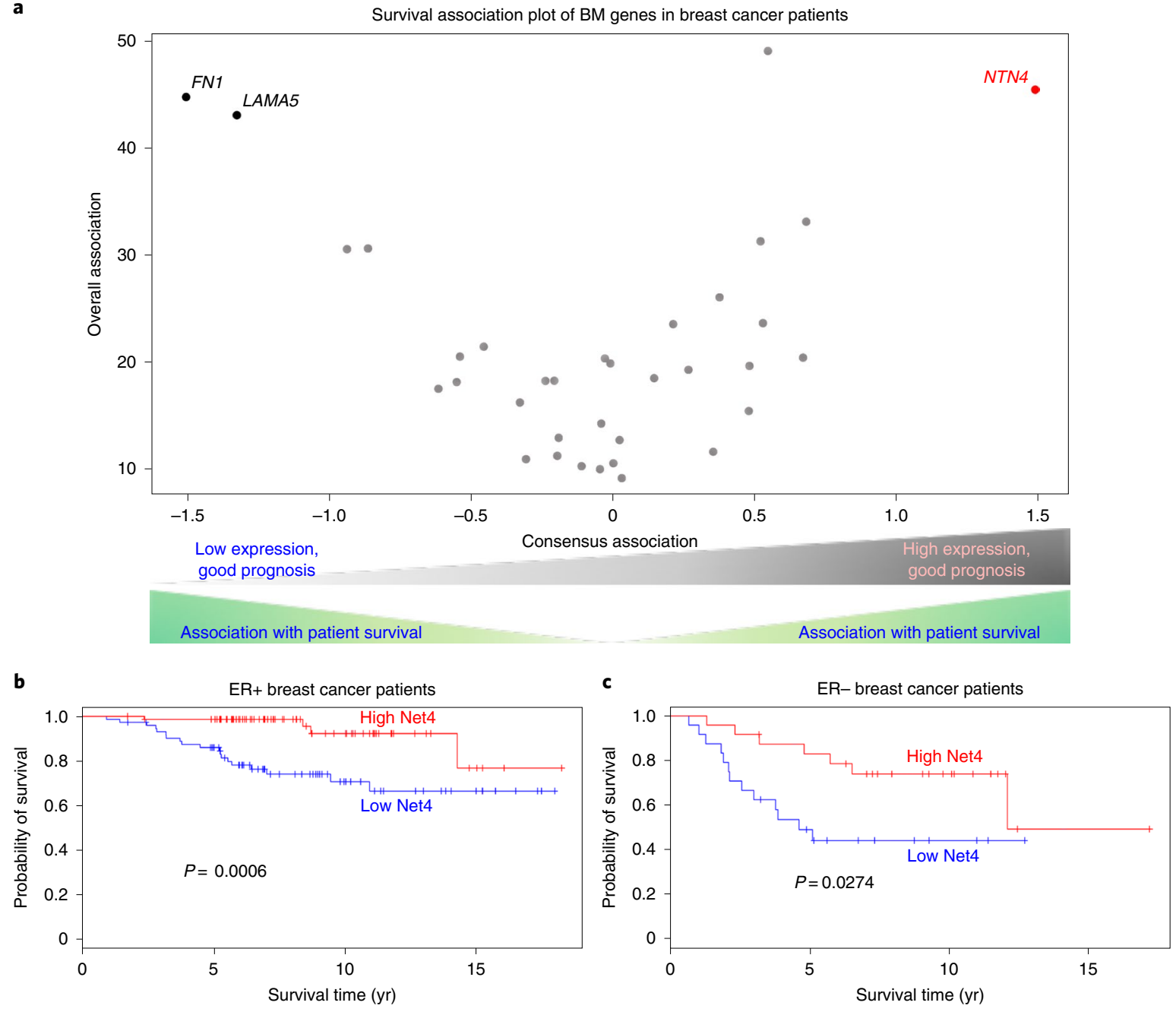

Fig. 1 Association of the expression of genes encoding for BM proteins with breast cancer patient survival. a, For each of 35 BM genes present in the NKI dataset, Cox proportional hazard models were used to compute 28 statistics for association with survival:death and metastasis, in the Normal component and the Disease component, in seven breast cancer subgroups: ER positive; ER negative; HER2 overexpressing; HER2 overexpressing, ER positive (luminal B); HER2 overexpressing, ER negative; HER2 normal, ER negative (basal-like); and HER2 normal, ER positive (luminal A). For the 28 groups we computed an overall association (the sum of the $\log _{10}$-transformed $P$ values) and a consensus association (the average of the $\log _{10}$-transformed $P$ values multiplied by +1 (association of high levels with good prognosis), -1 (association of low levels with good prognosis) or 0 (no association)). A plot of consensus association versus overall association shows low levels of fibronectin (FN1) and laminin $\alpha 5$ (LAMA5) as well as high levels of Net4 (NTN4) to be most consistently associated with good prognosis across most tumour groups (highest consensus association). FN1, LAMA5 and NTN4 reveal the best $P$ values overall (very high overall significance). Grey dots indicate BM genes with a low consensus association between -1.0 and 1.0. b,c, Kaplan-Meier survival (death) analysis: tumour group 1, low levels of NTN4 in the Normal component (33th percentile NTN4 levels) versus tumour group 2, high levels of NTN4 in the Normal component (67th percentile NTN4 levels). Oestrogen-receptor-positive tumours show a strong association $(P=0.0006)$ between high levels of NTN4 in the Normal component and good survival (b). Oestrogen-receptor-negative tumours show an association $(P=0.0274)$ between high levels of NTN4 in the Normal component and good survival (c).

high levels of the BM protein netrin-4 (Net4) as strongly associated with good prognoses. The more Net4 protein present within the $\mathrm{BM}$, the softer this barrier, resulting in reduced cancer cell invasion which is associated with increased patient survival. Mechanistically, Net4 binds to laminin, thereby opening the laminin ternary node complex, which in turn reduces the overall BM stiffness. We show that the global distribution of Net4 results in bigger pores within the BM and reduced BM stiffness. This article provides direct evidence that the BM mechanics are dominant over BM pore size in regulating cancer progression, and expands our understanding of BM transmigration. This detailed knowledge of the complex BM material additionally may form the basis of future therapeutic interventions to alter metastases formation.

\section{Results}

Association of BM gene expression with cancer patient survival. The BM presents a polymer barrier for cancer cells to breach and is therefore involved in many steps during the invasion-metastasis process ${ }^{3,4}$. Although the paradigm of BM transmigration is based on cell forces applied to this polymeric material ${ }^{10-12}$, we investigated the distinct concept that the architecture of the intact BM might apply physical forces on cancer cells directly, thereby altering their behaviour. The architecture of a material is crucially influenced by its composition. We generated a list of 49 genes encoding for BM components, which we determined from the literature and from our recent report $t^{5,13-20}$ (Supplementary Table 1). 
We first examined the extent to which BM components might be associated with survival in a public breast cancer dataset from the Netherlands Cancer Institute (NKI) consisting of 295 patients $^{21}$, and a dataset from 13 normal breast samples (three from reduction mammoplasties and ten from pathologically normal breast tissue from breast cancer patients) ${ }^{22}$. Here we investigated association with survival within multiple patient groupings, along several data components, and then computed a measure of association with survival for each BM gene across all these groupings. Of the 49 BM genes, 35 were present in the breast cancer dataset. The genomic data were first decomposed into Disease and Normal components using the Disease-Specific Genomic Analysis (DSGA) method ${ }^{22}$. Essentially, the Normal component of a tumour identifies the 'normal'-like profile of the tumour sample from a comparison with normal tissue data. By contrast, the Disease component of a tumour identifies the extent to which the actual tumour data deviate from all possible 'normal'-like signatures.

Figure la shows the plot of consensus versus overall associations for all BM genes present in the NKI dataset. Net4 (NTN4) exhibits both strong overall association and strong positive consensus association with survival. Laminin $\alpha 5$ (LAMA5) and fibronectin (FN1) likewise show strong overall association and strong negative association with survival (Fig. 1a). We wanted to further explore whether the association was most visible as a type of 'normal' BM present around the tumours (evidenced by the Normal component) or in the possible 'abnormal' modification of the BM in the tumour (evidenced by the Disease component). The Normal component's statistics show a much stronger association with survival than the Disease component or nonDSGA data (not decomposed by DSGA) (Extended Data Fig. 1a). We therefore focused primarily on the association with survival in the Normal component of the data. We concentrated our attention on Net 4 because of the availability of a viable knockout mouse ${ }^{23}$ to study the impact of 'normal' BM independent of tumour-derived or tumour-induced Net4 levels within BMs on cancer progression, as fibronectin and laminin $\alpha 5$ knockout mice are embryonic lethal ${ }^{24,25}$. We investigated the association between Net 4 and survival in breast cancer patients with ER positive (ER+) or ER negative (ER-) tumours. High levels of Net4 (NTN4) were significantly associated with good prognosis in both subtypes (Fig. 1b,c). We further explored the association of NTN4 expression in the Normal component with survival in renal cell carcinoma ${ }^{26,27}$ using a cohort of 166 renal cell carcinomas together with 35 normal tissue samples. This analysis showed again that high levels of Net4 (NTN4) were significantly associated with good prognosis (Extended Data
Fig. 1b,c). Our analysis indicated that 'normal' BMs containing high amounts of Net 4 are associated with good prognosis in breast and kidney cancer patients.

BMs deficient for Net4 promote metastases formation. To analyse whether tumour progression is altered in mice lacking Net4 within BMs, we orthotopically injected mouse breast cancer E0771 cells into the mammary fat pad of Net 4 wildtype and knockout mice. After primary tumours had reached their maximum size, we resected them in all mice on the same day, and allowed metastases to develop. We analysed lung tissues $28 \mathrm{~d}$ postresection (Fig. 2a). The primary tumour weight was similar in both genotypes on the day of resection, indicating that loss of Net4 does not influence primary tumour growth (Fig. 2b). Quantification of lung metastases revealed that loss of Net4 significantly increased the number of metastases (Fig. 2c-e). We investigated the impact of Net4 on stromal cell infiltration, cancer cell proliferation and fibroblast activity to exclude that Net4 directly affects cell behaviour, as Net4 is reported to possess protumorigenic ${ }^{28-33}$ and antitumorigenic ${ }^{34-37}$ activity. Our data demonstrated that loss of Net 4 in the host does not alter the abundance of CAFs and immune cells (macrophages and $\mathrm{T}$ cells) in E0771 primary tumours (Extended Data Fig. 2a). Additionally, recombinant Net 4 slightly promoted the proliferation of mouse breast cancer cells, but not human breast cancer cells or mouse melanoma cells (Extended Data Fig. 2b). However, primary tumour growth was not altered in Net4 wildtype and knockout mice, and formation of lung metastases was significantly increased in mice deficient in Net4. Therefore, these data indicate an additional direct activity of Net4 on cells. Furthermore, low and high doses of Net4 did not influence the contractile ability of CAFs (Extended Data Fig. 2c). These results suggest that the increase in lung metastases in the Net 4 knockout mouse cannot be attributed to the loss of direct effects of Net 4 on cells.

Therefore, we next investigated whether BMs with or without Net4 alter cancer cell invasion. To this end, we performed a three-dimensional (3D) spheroid invasion assay in a reconstituted BM matrix ${ }^{38}$ (rBM, Matrigel). To mimic the BM from Net4 knockout and wildtype animals, we embedded breast cancer spheroids into rBM and into rBM supplemented with recombinant Net4 as well as a Net4 mutant protein, substantially decreasing its laminin-binding capacity (Mut), which we recently generated and validated $^{39}$. Cells invaded rBM and rBM supplemented with the Mut significantly more than into their counterparts supplemented with wildtype Net4 (Fig. $2 \mathrm{f}$ and Extended Data Fig. 3a), indicating a laminin binding-related mechanism. Interestingly, addition of the same

Fig. 2 | Net4-deficient alveolar BM favours metastases formation. a, Experimental design of orthotopic injection of E0771 mouse breast cancer cells into the mammary fat pad of Net4 wildtype (WT) and knockout (KO) mice followed by primary tumour resection and subsequent analysis. $\mathbf{b}$, Box-and-whisker plot of the primary tumour weight on the day of resection (two-tailed unpaired $t$ test, minimum to maximum, median is shown as line, mean is displayed as cross, data points appear as grey dots; $n=9(\mathrm{WT}), n=12(\mathrm{KO}) ; t=0.4581$, degrees of freedom (d.f.) $=19)$. NS, not significant. c, Representative H\&E images of lungs from Net4 WT and Net4 KO mice $28 \mathrm{~d}$ postresection of the primary E0771 tumour. Scale bar, 3 mm. d, Box-and-whisker plot of macroscopic lung metastasis number in mice injected with E0771 cells into the mammary fat pad of Net4 WT and Net4 KO (two-tailed Mann-Whitney test for all analyses, minimum to maximum, median is shown as line, mean is displayed as cross, data points appear as grey dots; $n=9$ (WT), $n=12$ $\left.(\mathrm{KO}) ;{ }^{\star} P<0.05\right)$. e, Box-and-whisker plots of microscopic lung metastases (left) and total metastasis area (right) of mice injected with E0771 cells into the mammary fat pad of Net4 WT and Net4 KO (two-tailed Mann-Whitney test for all analyses, minimum to maximum, median is shown as line, mean is displayed as cross, data points appear as grey dots; $n=9$ (WT), $n=12(\mathrm{KO})$; ${ }^{\star} P<0.05$, $\left.{ }^{\star \star} P<0.01\right)$. f, Representative images of MDA-MB-231 sphere invasion in Matrigel (control) and Matrigel containing 50\% Net4 (Net4) or 50\% Net4 laminin-binding mutant (Mut) molecules relative to laminin 111 within the Matrigel matrix. Scale bar, $100 \mu \mathrm{m}$. Right: scatter plot of sphere invasion (ordinary one-way ANOVA test, mean is displayed as black line; $n=27 ; F=94.2$, d.f. $\left.=78 ;{ }^{\star \star \star \star} P<0.0001\right)$. $\mathbf{g}$, Time schedule of intravenous injection of E0771 cells into Net4 WT and Net4KO mice and subsequent analysis $28 \mathrm{~d}$ postinjection. h, Representative H\&E images of lungs from Net4 WT and Net4 KO mice $28 \mathrm{~d}$ postinjection of EO771 cells. Scale bar, 3 mm. i, Box-and-whisker plot of macroscopic lung metastasis (two-tailed Mann-Whitney test, minimum to maximum, median is shown as line, mean is displayed as cross, data points appear as grey dots; $\left.n=12(\mathrm{WT}), n=10(\mathrm{KO}) ;{ }^{\star} P<0.05\right)$. j, Box-and-whisker plots of microscopic lung metastasis (left), area/metastasis (centre) and total metastasis area (right) of intravenous injection of E0771 cells into Net4 WT and Net4 KO mice after $28 \mathrm{~d}$ (two-tailed Mann-Whitney test, minimum to maximum, median is shown as line, mean is displayed as cross, data points appear as grey dots; $n=12$ (WT), $n=10$ $\left.(\mathrm{KO}) ;{ }^{\star \star} P<0.01,{ }^{\star \star \star} P<0.001\right)$. 
a

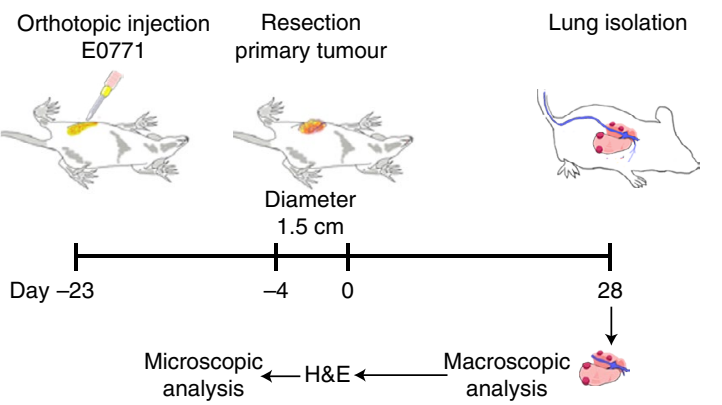

b

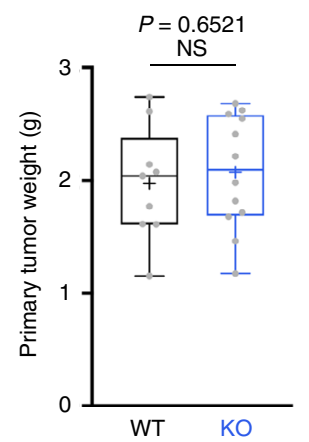

f
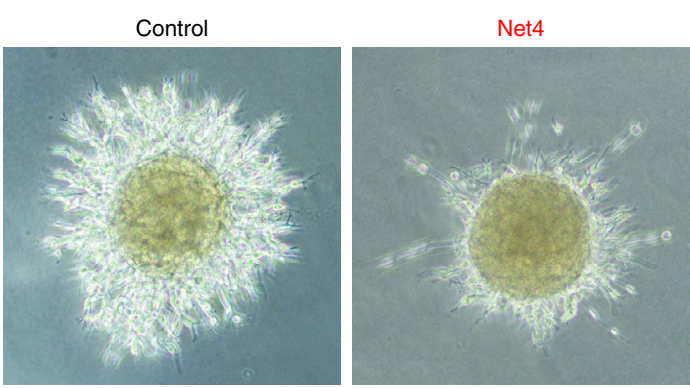

d
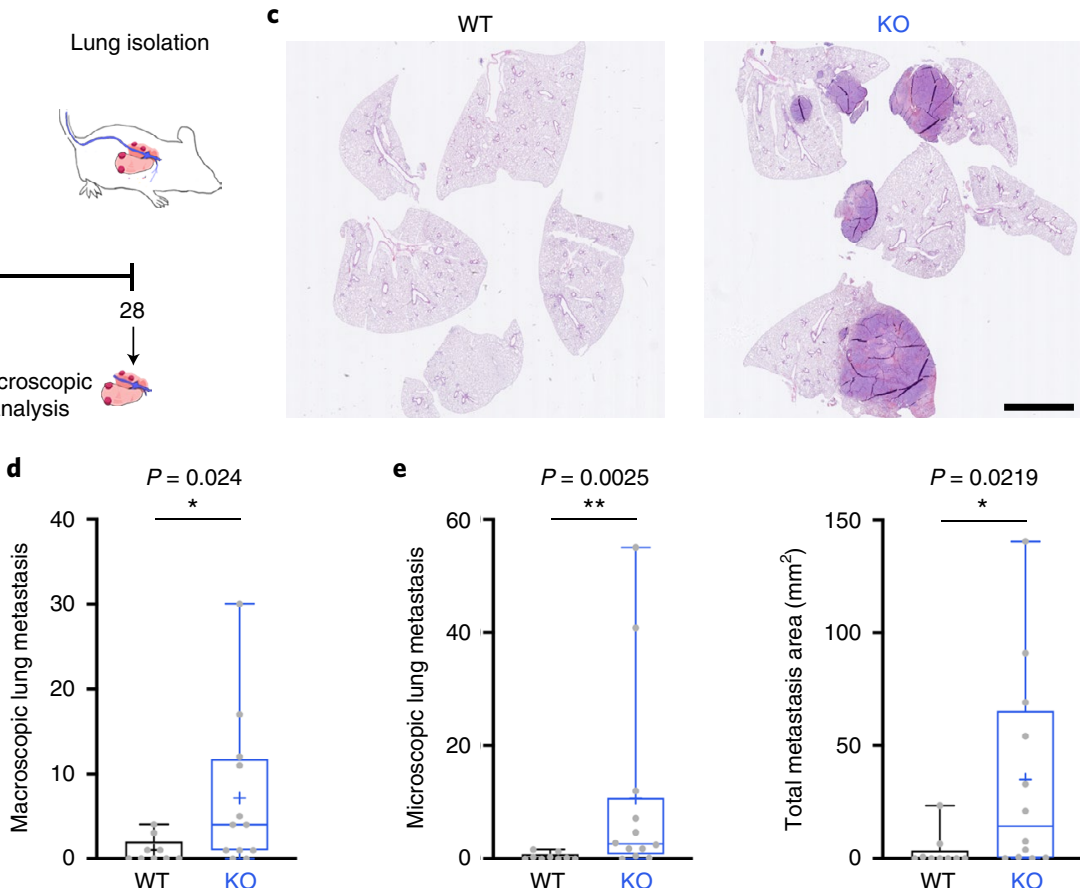

e
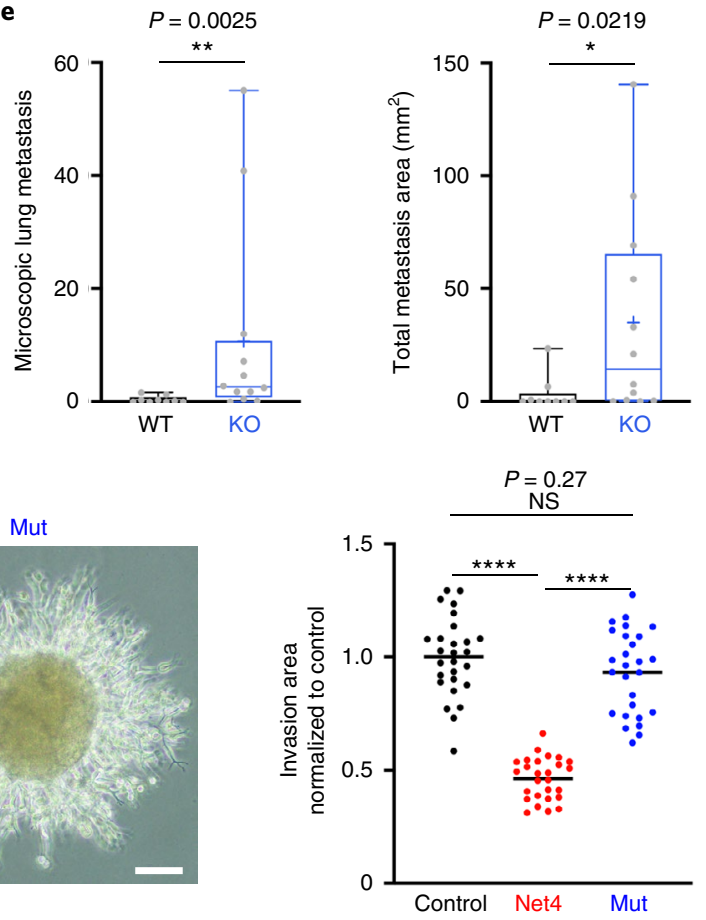

g

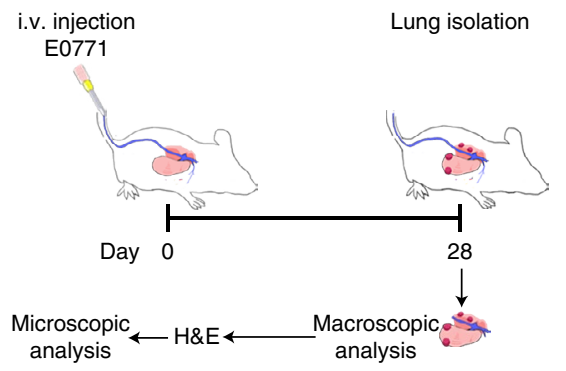

i

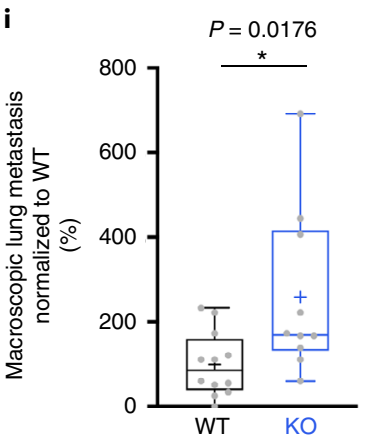

j

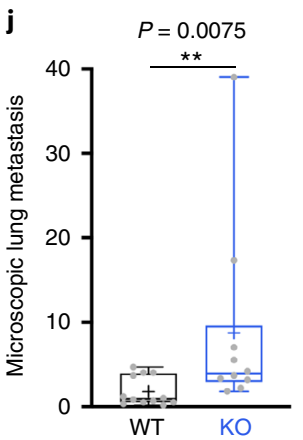

h

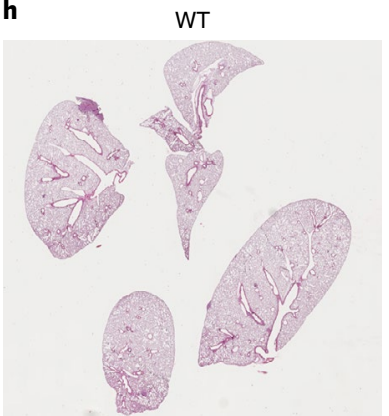

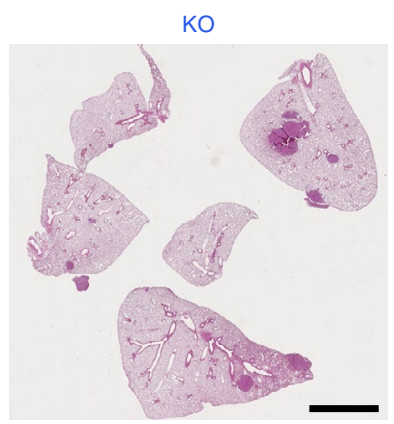
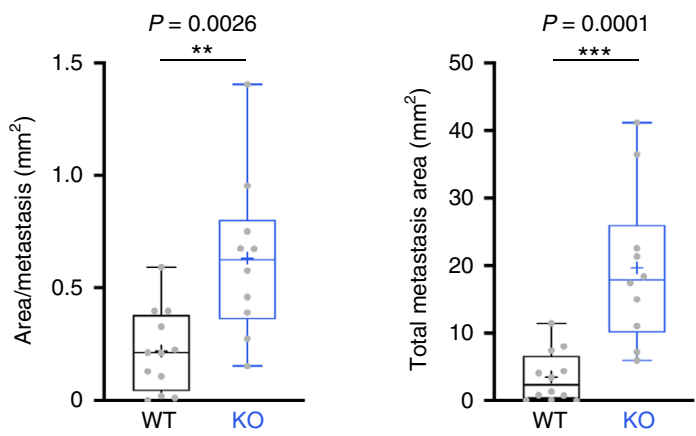
number of Net 4 molecules to breast cancer spheroids in a collagen I matrix promoted cell invasion in a manner independent of laminin binding (Extended Data Fig. 3b). These data indicate that BMs containing Net4 restrict the ability of breast cancer cells to move through the BM and additionally uncover a context-dependent proinvasive activity of Net4, in line with previous studies ${ }^{28-32}$.

To understand the impact of 'normal' Net4 expression on cancer progression, we determined Net4 localization in the alveolar BM, as this $\mathrm{BM}$ is the major barrier for cancer cells to breach in the experimental metastasis model. We decellularized lung tissues from Net4 wildtype and knockout ${ }^{23}$ mice using the in situ decellularization of tissues approach ${ }^{19,40}$, which preserves ECM architecture $^{19}$. Net4 was localized inside the alveolar BM (Extended Data Fig. 4a). Collagen IV and laminin had a similar distribution, arrangement and amount in Net4 knockout mice (Extended Data Fig. 4a). To determine whether there are developmental alterations in protein abundance between lung tissues from Net4 knockout and wildtype mice, we performed tandem mass tag (TMT) mass spectrometry analysis of lung tissues. Strikingly, no BM proteins apart from Net4 were altered between Net 4 knockout and wildtype mice (Extended Data Fig. $4 \mathrm{~b}$ and Supplementary Table 2). Thus, these data suggest that the Net 4 knockout mouse is ideal for studying the functional role of Net4 within native BMs during metastases formation. Intravenous injection of the E0771 cells (Fig. 2g) into Net4 wildtype and knockout animals resulted in a significant increase in lung tumour burden for all metastasis parameters (foci size, number and area) in animals with an alveolar BM lacking Net4 (Fig. $2 \mathrm{~h}-\mathrm{j}$ ).

Together, these data revealed that lung colonization is altered by Net4-containing BMs and further suggest that Net4-containing BMs impact the steps of the invasion-metastasis process ${ }^{3,4}$ where the BM plays a role (invasion, intravasation and extravasation).

We confirmed our findings in a second cancer model using HCmel12 mouse melanoma cells. Net4-containing BMs significantly decreased HCmel12 cancer cell invasion in spheroid invasion assays (Fig. 3a and Extended Data Fig. 5a). We intravenously injected HCmel1 2 cells into Net4 knockout and wildtype mice, isolated lung tissues and further inspected the abdomen for metastases in the peritoneum and mesentery ${ }^{41}$ (Fig. $3 \mathrm{~b}$ ). We observed a significant increase in metastases in the lung and the abdominal space in Net4 knockout mice (Fig. 3c,d).

To exclude that endogenous Net4 levels within cancer cells contribute to the alteration in lung metastatic colonization, we next performed quantitative real-time polymerase chain reaction
(rtPCR) to determine Net4 (Ntn4) expression levels in different cell lines. Ntn 4 expression was higher in EO771 cells than in HCmel12 cells (Extended Data Fig. 5b). We generated E0771 cells deficient for Net4 using CRISPR/Cas9 technology and repeated the metastasis studies in Net4 knockout and wildtype mice. The generated cells showed a significant increase in lung metastases (number, area and total metastases area) in Net4 knockout mice (Extended Data Fig. $5 \mathrm{c}-\mathrm{e})$, demonstrating these effects are independent of tumour-derived Net4.

Our results thus far suggest that BMs containing Net4 affect the formation of metastases. To investigate which cell types produce Net 4 in human cancer patients, we analysed a published single-cell RNA sequencing (scRNA-seq) dataset from 19 melanoma patients ${ }^{42}$. We assigned clusters to seven cell types based on cell-type-specific markers (Extended Data Fig. 6a), and found Net4 (NTN4) to be mainly produced by CAFs and endothelial cells (Fig. $3 e$ and Extended Data Fig. 6b). To determine whether CAFs and endothelial cells are the major source of BM components, we analysed the expression of $48 \mathrm{BM}$ components within all cell types (Supplementary Table 1). Indeed, CAF and endothelial cells express the major proportion of all BM components (Fig. 3f, Extended Data Fig. $6 c$ and Extended Data Fig. 7). Based on these findings that Net4 is produced by cell types shaping the BM, we determined the association of Net4 expression levels with prognosis in an available dataset with 424 melanoma patients ${ }^{43}$. High levels of NTN4 were indeed significantly associated with good prognosis of melanoma patients (Fig. 3g).

Net4 softens BMs. As most of the abdominal metastases in the HCmel12 model were attached to the mesenteric vessels, we focused on visualizing Net 4 within these vessels and confirmed the absence of Net4 in the vascular BM (vBM) of Net 4 knockout mice (Extended Data Fig. 8a), indicating a link between increased metastasis and absence of Net4. Mesenteric arteries are a good model to assess the BM stiffness ex vivo as they contain only a thin layer of vascular smooth muscle cells separated from the endothelial cell layer by the BM and internal elastic lamina ${ }^{44}$.

To determine the effect of Net 4 on the biomechanical properties of the vBM, we performed pressure myography on mesenteric vessels from Net4 wildtype and knockout mice. This revealed that lack of Net 4 does not alter the overall structure of the mesenteric vessels (Extended Data Fig. 8b,c). We also employed pressure myography to obtain wall stress and strain parameters to determine the vessel

Fig. 3 | Net4-deficient alveolar and mesenteric vessel BM favours metastases formation. a, Representative images of $\mathrm{HCmel12}$ cell sphere invasion assay in Matrigel (control) and Matrigel containing 50\% Net4 or 50\% Net4 laminin-binding mutant (Mut) molecules relative to laminin 111 within the Matrigel matrix. Scale bar, $100 \mu \mathrm{m}$. Right: scatter plot of sphere invasion (Kruskal-Wallis test, mean is displayed as black line; $n=26$; ${ }^{\star \star \star \star} P<0.0001$ ). b. Experimental design of intravenous injection of $\mathrm{HC}$ mel12 mouse melanoma cells into Net4 WT and Net4 KO mice and subsequent analysis of lungs and abdomen 20 d postinjection. c, Representative images of lung lobes from Net4 WT and Net4 KO mice. Black spots on the lung surface are macroscopic metastases. Scale bar, $5 \mathrm{~mm}$. Right: box-and-whisker plot of macroscopic lung metastases in Net4 WT and Net4 KO mice (two-tailed Mann-Whitney test, minimum to maximum, median is shown as line, mean is displayed as cross, data points appear as grey dots; $n=32(\mathrm{WT}), n=28(\mathrm{KO})$; $\left.{ }^{\star \star \star \star} P<0.0001\right)$. d, Representative images of the peritoneal space from Net4 WT and Net4 KO mice. Black spheroids are abdominal metastases. Scale bar, 10 mm. Right: box-and-whisker plot of macroscopic abdominal metastases in Net4 WT and Net4 KO mice (two-tailed Mann-Whitney test, minimum to maximum, median is shown as line, mean is displayed as cross, data points appear as grey dots; $n=11$ (WT), $n=14\left(\right.$ KO); $\left.{ }^{\star \star} P<0.01\right)$. e, Violin plots of NTN4 expression for each cell type using scRNA-seq from 19 melanoma tumour patients ${ }^{42}$. The expression levels of NTN4 were quantified as $\log _{2}($ TPM/10 +1$)$, where TPM refers to transcript per million for NTN4. f, Violin plots of the expression of the 49 BM genes for each cell type. The summed $z$ scores of the 49 BM genes (BMREP) were used as representative expression values for each cell. $\mathbf{g}$, Kaplan-Meier plot showing the difference of overall survival between melanoma patients with high (67th percentile NTN4 levels) and low (33th percentile NTN4 levels) NTN4 expression calculated by a log rank test $(P=0.0008)$. $\mathbf{h}$, Stress-strain graph of pressure myography analysis of mesenteric vessels from Net4 WT and Net4 KO mice (linear regression test, single line for each dataset; $n=8$; ${ }^{\star} P<0.05$ ). $\mathbf{i}$, Experimental design of rescue approach, in which Net4 KO mice were treated without (control) and with $2.5 \mathrm{mg}$ per kg body weight recombinant Net4 (Net4) intraperitoneally injected for five consecutive days. Three days after treatment was stopped (day 8 ), $\mathrm{HC}$ el12 cells were intravenously injected. Macroscopic lung metastases were analysed $20 \mathrm{~d}$ postinjection. $\mathbf{j}$, Representative images of lung tissue from control and Net4-treated (Net4) Net4 KO mice. Black spots on the lung surface marked with arrowheads are macroscopic metastases. Scale bar, $5 \mathrm{~mm}$. Right: box-and-whisker plot of macroscopic HCmel12 lung metastases in control and Net4-treated (Net4) Net4 KO mice (two-tailed Mann-Whitney test, minimum to maximum, median is shown as line, mean is displayed as cross, all data points appear as grey dots (ctrl) and red dots (Net4); $\left.n=18 ;{ }^{\star} P<0.05\right)$. 
a

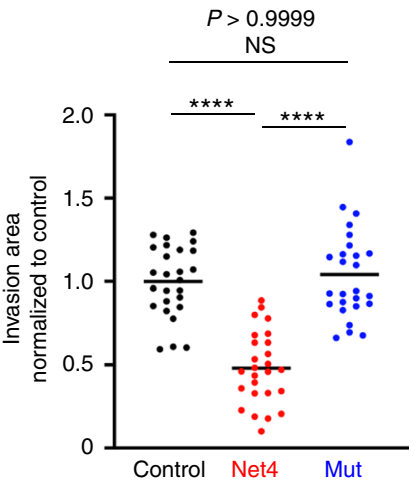

c
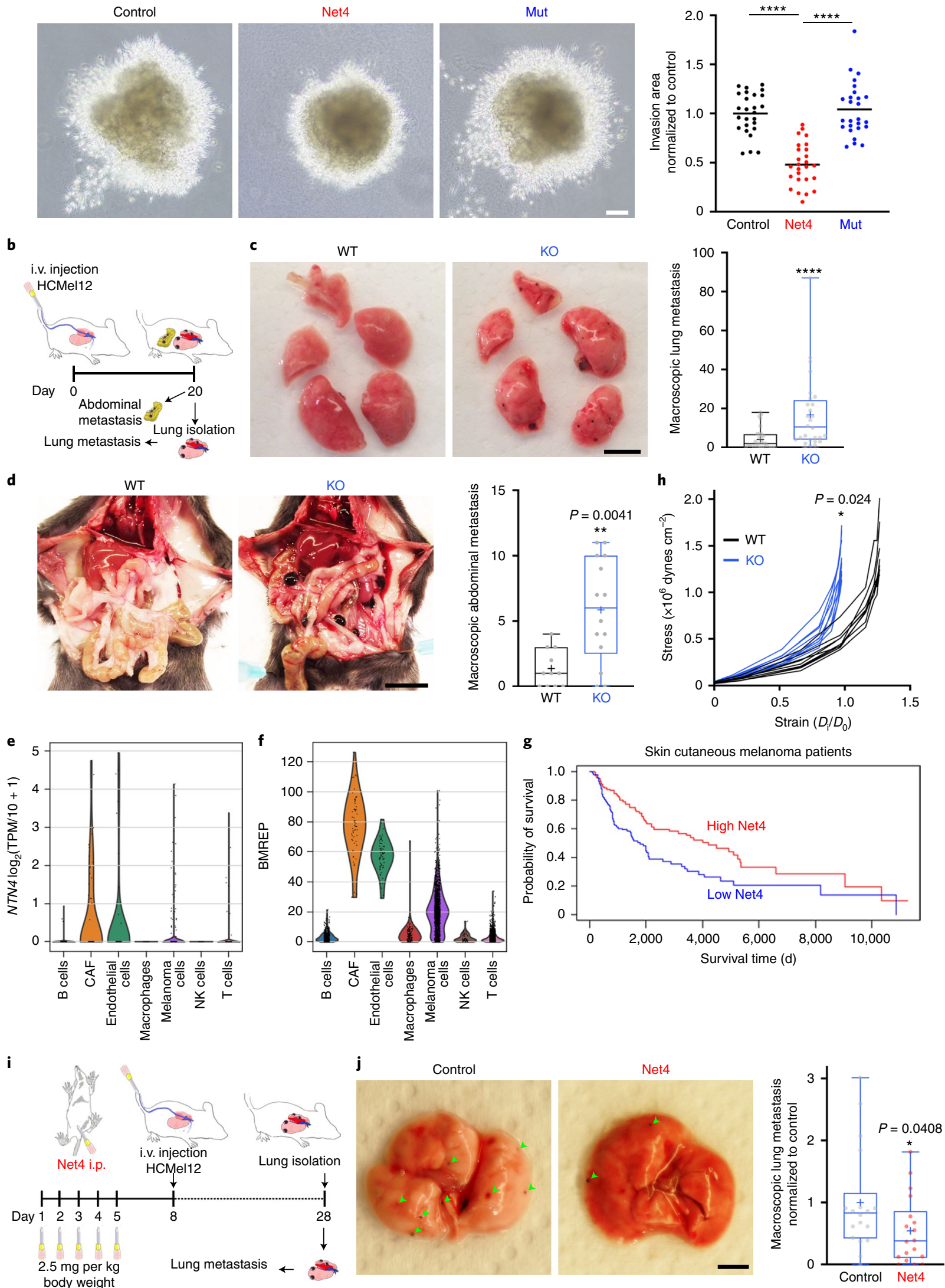
stiffness. This analysis revealed a leftward shift of the stress-strain curve in Net 4 knockout compared with wildtype mice, indicating increased stiffness in mesenteric vessels (Fig. 3 h).

Next, we sought to investigate the impact of reapplying Net4 to Net 4 knockout mice on the formation of metastases. Here, we treated Net4 knockout mice for five consecutive days intraperitoneally with $2.5 \mathrm{mg}$ per $\mathrm{kg}$ body weight recombinant Net 4 before intravenous injection of $\mathrm{HCmel} 12$ cells, $3 \mathrm{~d}$ after the treatment was stopped (Fig. 3i). Strikingly, the systemic injection of Net4 significantly reduced the formation of lung metastases (Fig. $3 j$ ).

As the knockout mice harboured more lung metastases, we next investigated the biomechanical properties of the alveolar BM. There is a growing literature of $\mathrm{BM}$ mechanics ${ }^{8,12,45-49}$ yet no measurements of alveolar BM. To keep the native tissue properties preserved we used rehydrated, cryoembedded lung tissues for the atomic force microscopy (AFM) analysis (Fig. 4a), because BM biomechanics are substantially altered in a dehydrated state ${ }^{45}$.

The alveolar BM is localized between an epithelial and endothelial cell layer. To identify the alveolar BM stiffness, we developed a tailored experimental approach where the AFM tip is moved across the alveolar space from the epithelial side to the endothelial side, crossing the alveolar wall and the BM (Fig. 4b). Data acquisition over this distance resulted in a Young's modulus pattern that enabled identification of the BM in between the softer cell layers (Fig. 4b). Using this method, we analysed Net 4 knockout and wildtype mice alveolar BM stiffness and found BM to be significantly softer in the presence of Net4 (25 kPa for wildtype versus $50 \mathrm{kPa}$ for knockout mice). These data, together with the pressure myography analysis of mesenteric vessel stiffness, indicated that Net4-containing BMs are generally softer than BMs lacking Net4 (Fig. 4b) and that BM mechanics determined the stiffness of the entire vessel.

Next, we set out to confirm the altered BM stiffness in Net4 knockout animals at a cellular level in vivo. Discher and colleagues recently postulated that caveolae structures are formed on soft matrix and are less prominent on stiff matrix ${ }^{50,51}$ (Fig. 4c). Therefore, we employed transmission electron microscopy to assess caveolae structures in the capillary endothelium of alveoli in Net4 knockout and wildtype mice. We observed clear omega-shaped caveolae structures in wildtype mice after fusion with the plasma membrane. However, caveolae-forming structures in the Net4 knockout mice were mainly intracellularly localized, and the number of omega-shaped alveolar BM-fused caveolae was significantly decreased (Fig. 4d). Therefore, we analysed the ratio of alveolar BM-fused to non-fused caveolae. This analysis revealed a significant decrease in the fused to non-fused caveolae ratio in Net 4 knockout mice (Fig. 4d). These data support our AFM analysis and pressure myography analysis that found a strong correlation between Net4 presence and BM stiffness (Fig. 3h and Fig. 4b). Moreover, our data suggest that caveolae structures might be useful markers to indicate BM stiffness alterations in vivo.
To evaluate whether Net4 expression is associated with tissue stiffness in cancer patients, we analysed a previously published dataset from human high-grade serous ovarian cancer metastasis ${ }^{52}$. NTN4 was the gene most strongly associated with omental metastasis tissue softness across 31 patient samples (Fig. 4e) and therefore strongly correlates negatively with tumour tissue stiffness (Fig. $4 \mathrm{f}$ and Supplementary Table 3). These data suggest that BM organization, and likely its stiffness, may be clinically important in different types of cancer. We further noted that NTN4 is negatively correlated with the expression of malignant cell markers (PAX8 and EPCAM), and positively correlated with BM-encoding genes (Extended Data Fig. 9a-c and Supplementary Table 4). This suggests that the impact of Net4 is based on its localization within the BM. Moreover, the $\mathrm{BM}$ seems to have a similar impact on breast and ovarian cancer patients (Extended Data Fig. 9d).

Laminin ternary node complexes determine BM stiffness. To explore whether Net4 protein levels directly affect BM stiffness, we investigated the stiffness of rBM supplemented with Net4. We performed AFM analysis following two different approaches: premixing of Net4 with rBM before polymerization; and titration of Net4 to the already polymerized rBM (Fig. 5a and Extended Data Fig. 10a). Titration of only $0.6 \%$ of Net 4 proteins in relation to laminin molecules within the rBM caused a 20\% decrease of the rBM Young's modulus compared with a lack of Net 4 (Fig. 5b). Addition of $25 \%$ Net 4 protein to the rBM resulted in a decrease of 50\% in rBM Young's modulus. These experiments revealed a strong initial reduction in BM stiffness until approximately $25 \%$ of Net 4 , then the stiffness starts to saturate, reaching $35 \%$ of the original Young's modulus at $50 \%$ of Net 4 (Fig. $5 b$ and Extended Data Fig. 10b,c). Thus, Net4 is indeed able to precisely decrease the stiffness of a BM in a concentration-dependent manner. Moreover, the alteration of the ternary node complex within the laminin network influences the overall stiffness of the BM. As the BM is built of two linked supramolecular networks comprised of collagen IV and laminin ${ }^{5,38}$, the observed saturation of the stiffness of the rBM at around $50 \%$ addition of Net 4 might be due to the stiffness of the unaffected collagen IV network.

We recently showed that cancer cells respond to ECM stiffness through adaptation of their intracellular viscoelasticity ${ }^{53}$. We analysed the intracellular viscoelasticity of cancer cells in $\mathrm{rBM}$ and $\mathrm{rBM}$ supplemented with Net4 as well as Mut protein using optical tweezers. Cancer cells significantly adapted their intracellular viscoelasticity to the softer matrix in rBM plus Net4 but not in rBM plus Mut protein (Extended Data Fig. 10d,e). These data strongly support that Net4 is altering ECM stiffness.

In order to rationalize our experimental findings, we introduced a coarse-grained model for the BM elastic properties that approximates the laminin network as an ordered two-dimensional honeycomb lattice. The dilution fraction $\phi$ quantifies the fraction of occupied to the total number of available Net4 binding sites; each

Fig. 4 | Influence of Net4 on the mechanical properties of the BM. a, Experimental design of AFM analysis of the alveolar BM from Net4 WT and Net4 KO mice. b, Representative AFM measurements to determine the stiffness (Young's modulus) of the alveolar BM in Net4 WT and KO mice. The AFM starts in one alveolus and goes to another, detecting different stiffness patterns corresponding to the alveolar space (S), followed by cell layer (C), followed by the BM (BM), followed by cell layer (C), ending in the opposite alveolar space (S). Right: box-and-whisker plot of the Young's modulus of the pulmonary BM from Net4 WT and Net4 KO mice (two-tailed unpaired $t$ test, minimum to maximum, median is shown as line, mean is displayed as cross, data points appear as grey dots; $n=8 ; t=2.529$, d.f. $\left.=14 ;{ }^{\star} P<0.05\right)$. $\mathbf{c}$, Scheme of caveolae structures inside capillary endothelial cells underlying a soft (fused) or stiff (non-fused) BM in the lung aveoli. d, Representative electron microscopy images of the alveolar BM from Net4 WT and Net4 KO mice. Blue arrows indicate caveolae structures within the capillary endothelium (CE) fused with the plasma membrane directed to the BM. Blue arrowheads indicate non-fused caveolae structures. AE, alveolar epithelium; E, erythrocyte. Scale bars, $250 \mathrm{~nm}$ and $100 \mathrm{~nm}$ (insets). Right: box-and-whisker plot of the ratio of fused to non-fused caveolae structures in Net4 WT and KO mice (two-tailed unpaired $t$ test, minimum to maximum, median is shown as line, mean is displayed as cross, data points appear as grey dots; $n=4$ (mice); ${ }^{\star \star \star} P<0.001$ ). e, Heatmap illustrates row $z$ scores of the log 2 c.p.m. (counts per million) gene expression of indicated genes. Samples (columns) ordered by increasing tissue stiffness (CANBUILD dataset-GSE71340 (ref. ${ }^{52}$ )). Genes (rows) arranged by increasing Pearson's correlation coefficient ( $r$ ) (Supplementary Table 3). f, Correlation between the Net4 (NTN4) gene expression and patient's tumour tissue stiffness (Pearson's $P=1.79 \times 10^{-7}, r=-0.78, n=31$ ). 
bound Net 4 removes a node in the laminin lattice. We modelled the elastic bonds as harmonic springs and determined equilibrium network structures for varying $\phi$ by energy minimization. Snapshots of networks without strain (Fig. 5c) and under a uniaxial strain of $40 \%$ (Fig. 5d) are shown. Diluted strained networks deform to minimize the number of highly stretched bonds (shown in red), giving rise to large pores with deformed bonds concentrated around the pore edges (Fig. 5d). Figure 5e shows calculated stress-strain profiles for two different dilutions. The curve for $\phi=10 \%$ revealed a soft floppy mode ${ }^{54}$, where up to a threshold strain of $\epsilon=7 \%$ no stress arises,
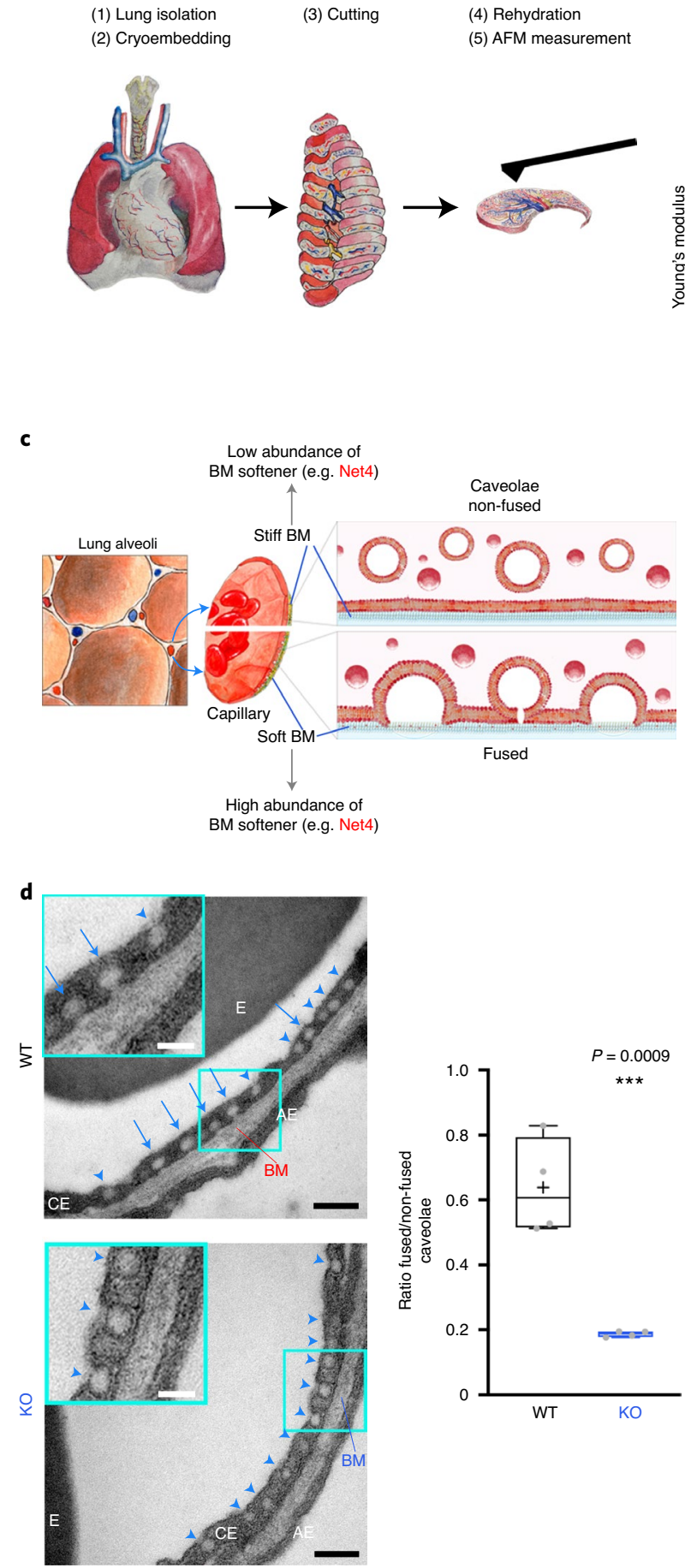

b
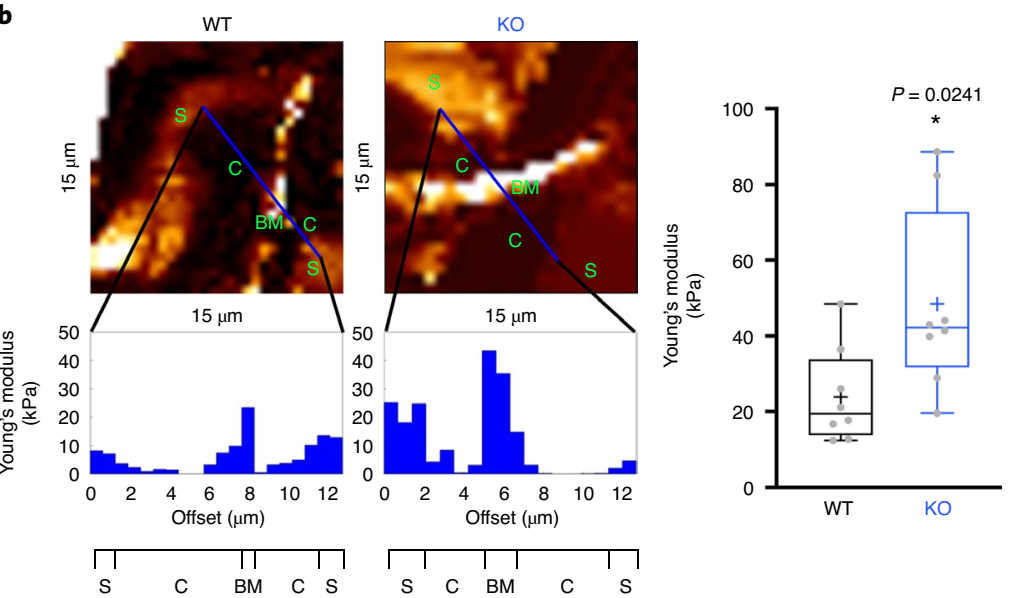

e
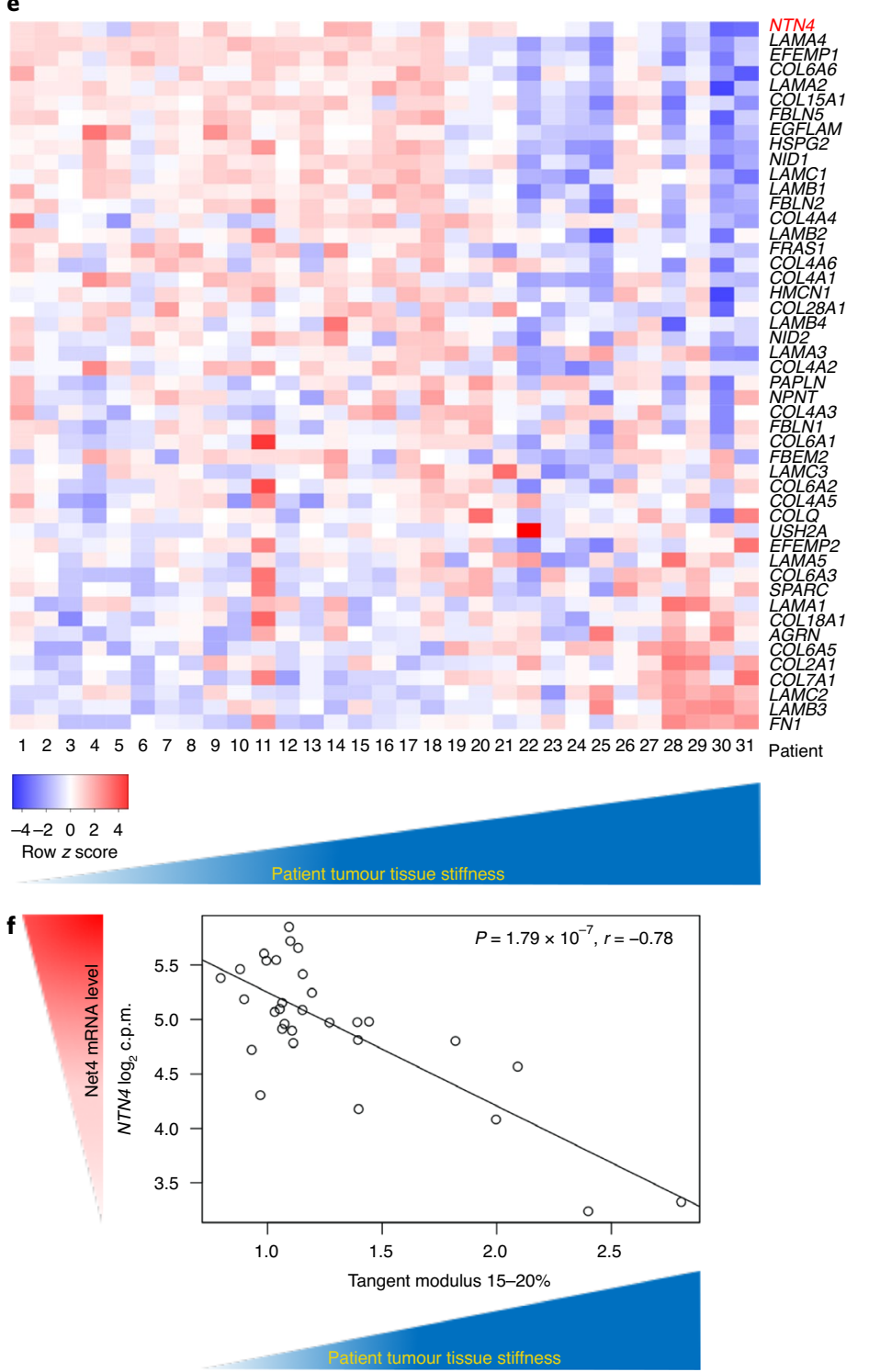
in contrast to the undiluted case $\phi=0$, which exhibited a linear stress-strain relation. The floppy behaviour of the diluted lattice is caused by the node rearrangement around the pores, which allows the system to accommodate large strains at minimal energetic cost
(Fig. 5c,d). The threshold strain increases roughly linearly with dilution (Extended Data Fig. 10f).

To account for the three-dimensional network deformation, as realized in the AFM experiments, we estimated the maximal a

Mixing

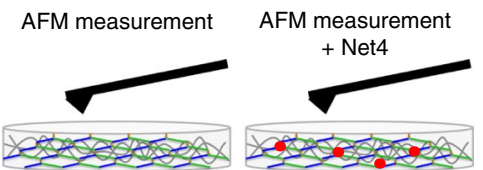

b

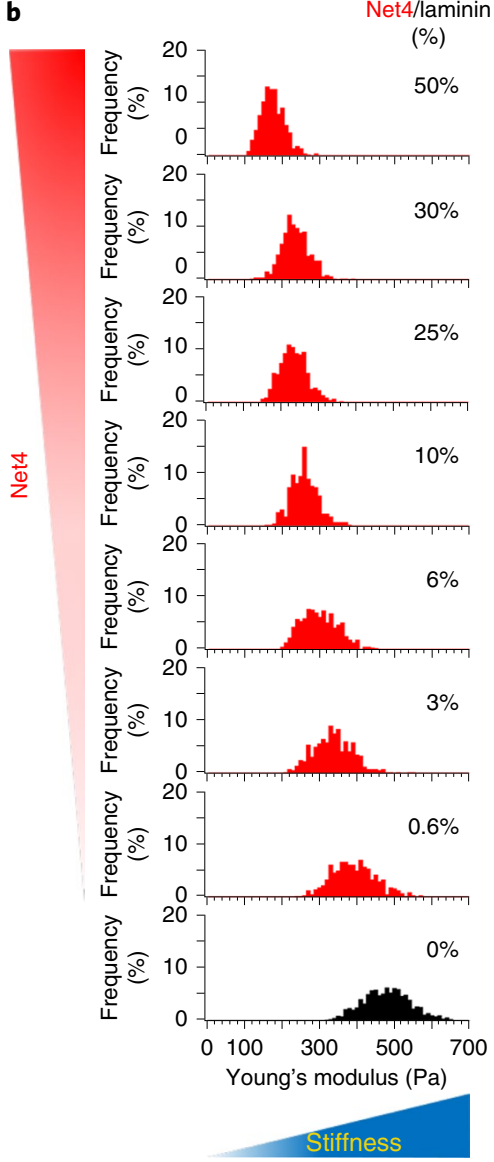

C

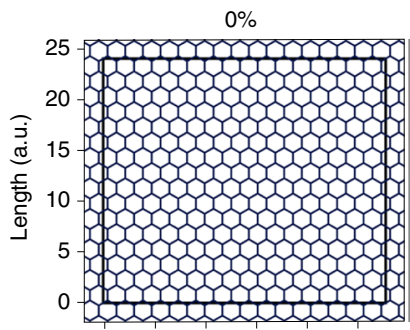

d

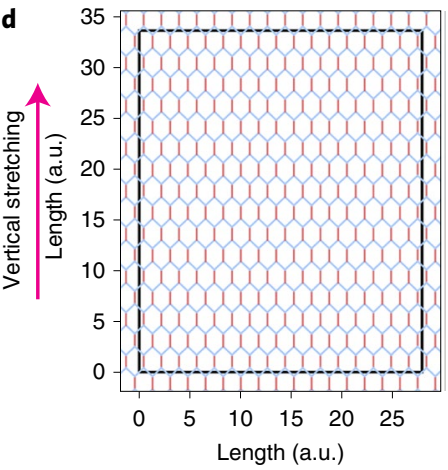

e
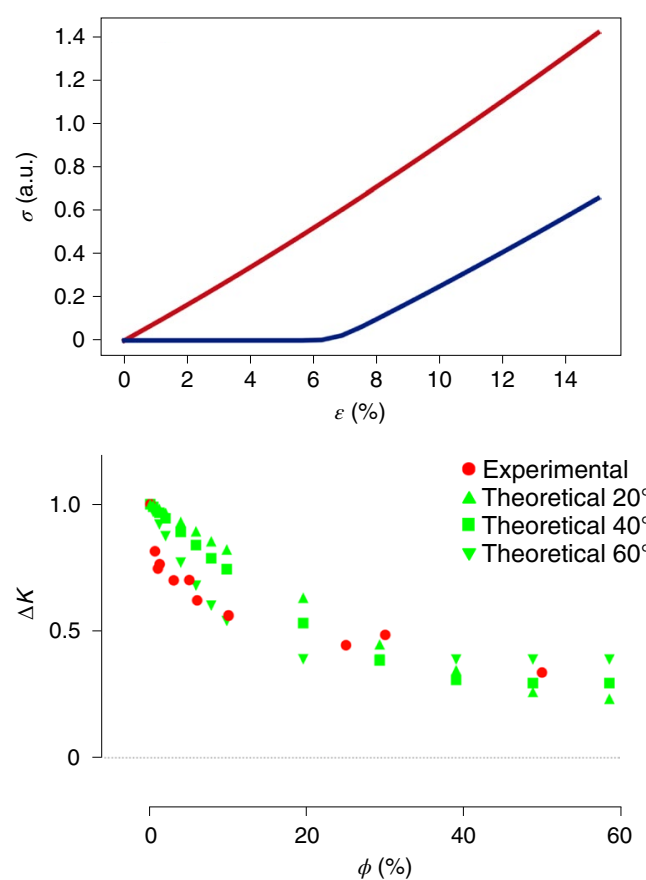

$10 \%$
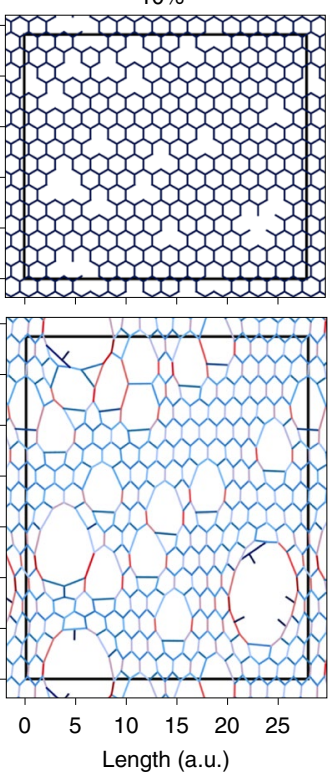

$20 \%$

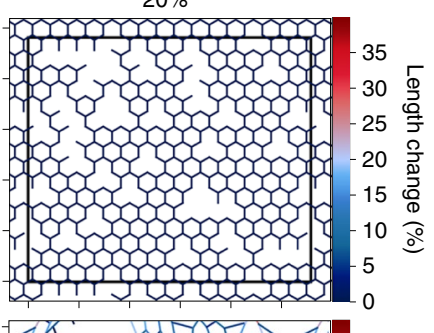

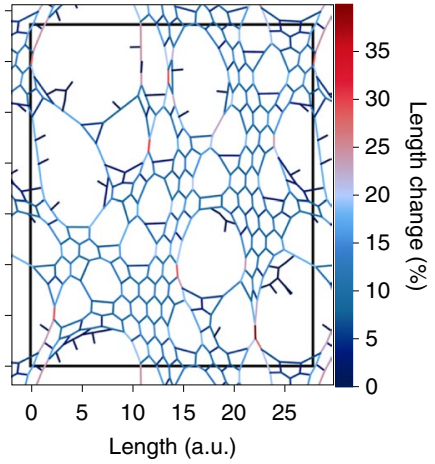

f

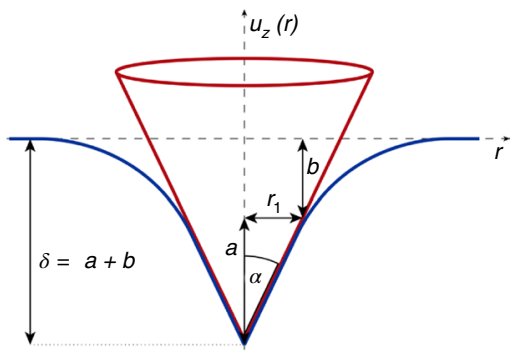

i

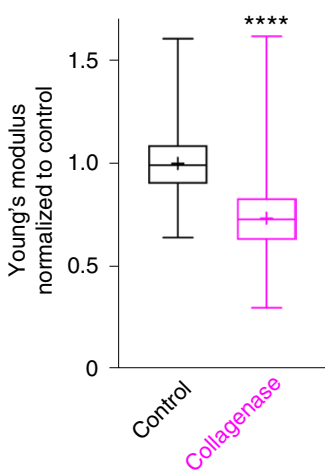

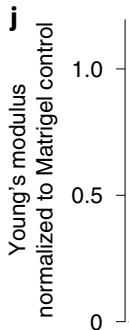
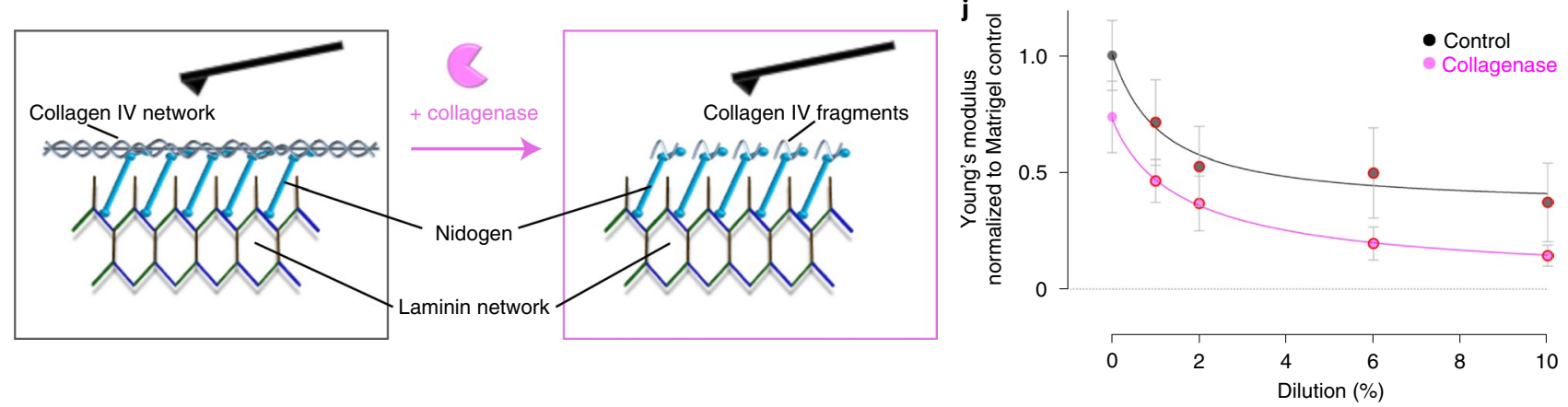
Fig. 5 | Decrease of BM stiffness via disrupting ternary laminin node complexes. a, Scheme of Young's modulus measurement by AFM of Matrigel and Matrigel mixed with Net4 (red dots). b. Histograms reveal the Young's modulus of measurements of pure Matrigel (black) and different Net4 percentages mixed into Matrigel (red). The amount as a percentage of Net4 molecules mixed into Matrigel indicates the ratio between Net4 and laminin 111 molecules. c,d, Simulation snapshots for no dilution, $10 \%$ dilution and 20\% dilution: unstrained diluted system (c); diluted system under $40 \%$ strain (d). e, Stress $(\sigma)$ versus strain $(\varepsilon)$ relations for the model laminin network at dilutions $\phi=0 \%$ (red) and $\phi=10 \%$ (blue). f, Sketch of the surface of an isotropic elastic medium (blue) deformed by a cone (red). Definitions of indentation depth $\delta$, half-opening angle $\alpha$ and contact radius $r_{1}$ are shown. $\mathbf{g}$, Relative stiffness change $(\Delta K)$ of Matrigel plotted against dilution $(\phi)$ obtained by AFM measurements of Net4 mixed into Matrigel (experimental) and data of the computational simulation (theoretical) for different values of the half-opening angle $\alpha$ of the conical indenter. One Net 4 molecule was estimated to open one laminin ternary node complex and the ratio between laminin and Net4 molecules or number of openings in relation to total nodes reveals the dilution. h, Scheme of the AFM experiment to determine the Young's modulus of Matrigel and Matrigel mixed with collagenase (indicated as a three-quarter circle shape) followed by titration of recombinant Net4. i, Box-and-whisker plot of Young's modulus of untreated (control) and collagenase-treated Matrigel (two-tailed Mann-Whitney test, minimum to maximum, median is shown as line, mean is displayed as cross; $n=569$ (control), $n=494$ (collagenase); $\left.{ }^{\star \star \star \star} P<0.0001\right)$. j, Young's modulus of untreated (control) and collagenase-treated Matrigel with different concentrations of Net4 presenting the dilution of the laminin network as a percentage. All values are normalized to Matrigel control at $0 \%$ Net 4 and include the standard deviation.

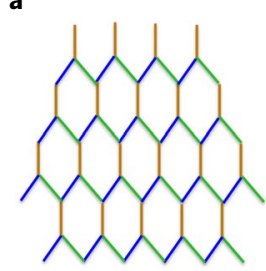

Modulation of BM mechanics
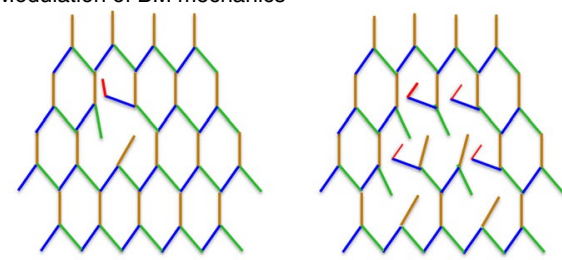

Net4

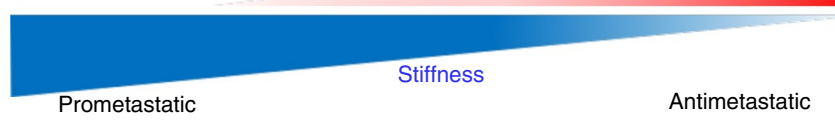

b
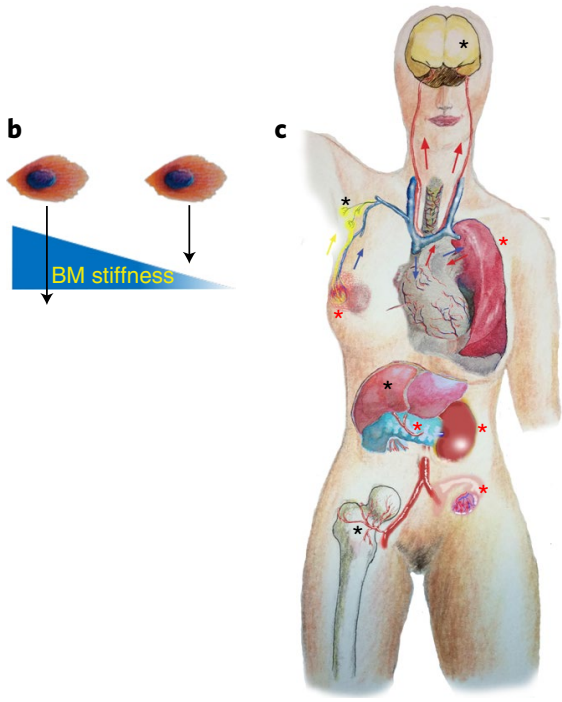

Fig. 6 | Impact of BM mechanics on metastasis. Our study reveals the impact of BM mechanics modulation on metastases formation and patient survival. $\mathbf{a}$, The scheme depicts the modulation of the BM through the ECM protein Net4 (red sticks) and how increasing amounts of Net4 reduce the stiffness of a laminin network (ternary node complex through the interaction of laminin $\alpha 1,2,3 B$ and 5 (yellow), $\beta 1,2$ and 3 (green), and $\gamma 1$ and 3 (blue)) and thereby of the entire BM. We show that stiff BMs favour metastases formation, and the softer a BM gets, the more antimetastatic it becomes. b. Our model shows that increasing BM stiffness is associated with increasing cancer cell invasion. c, Vessels present the major route for cancer cells to spread throughout the body to colonize secondary sites and establish metastases. Our data highlight that the BM stiffness defines cancer cell intravasation from breast, kidney and ovarian tissues and extravasation into lungs and mesentery (red asterisks). Although Net4 is not the defining factor for BM stiffness in all tissues, we hypothesize that BM stiffness is a key determinant also in these tissues including common metastatic sites such as lymph nodes, brain, liver and bone (black asterisks). extensional strain of an isotropic elastic medium that is deformed by a cone (Methods), as sketched in Fig. 5f. We included the constant elastic response from the collagen IV background, which added a single fit parameter to our model (Methods). Figure $5 \mathrm{~g}$ compares the theoretical rescaled deformation modulus $\Delta \kappa(\phi)=\kappa(\phi) / \kappa(0)$ for three different half-opening angles $\alpha$ of a conical indenter as a function of dilution to the experimental AFM data. Although a value of $\alpha=20^{\circ}$ best describes the shape of the pyramidal tip used in the AFM experiments, presumably higher values of $\alpha$ are closer to reality, and reflect structural imperfections of the AFM tip and the sample surface as well as torsional and radial strain effects. The background collagen elastic response accounts for the finite value of $\Delta \kappa(\phi)$ for large $\phi$. The quite abrupt BM stiffness decrease at low dilutions was well reproduced by the model, especially for $\alpha=60^{\circ}$ (Fig. $5 \mathrm{f}$ and Methods), and can be explained by the non-linear floppy elastic response of diluted laminin networks. This suggests that the mechanism by which Net4 softens BM consists of a systematic dismantling of the laminin network. Treatment of the Matrigel with collagenase (Fig. 5h) revealed a significant decrease of Matrigel stiffness of approximately 27\% (Fig. 5i) and an almost total loss of stiffness at around $10 \%$ of laminin node dilution (Fig. 5j). These data reveal that the background stiffness we measured is mainly based on the collagen IV network.

\section{Discussion}

ECM stiffness has long been known to be a major influencer of cancer metastasis; however, previous studies have focused on interstitial matrix-the $\mathrm{BM}$, and its contribution to matrix stiffness, has not been explored. Moreover, the functional role of the $\mathrm{BM}$ architecture during cancer progression has also not previously been investigated. Here we show that Net4 softens the BM in a laminin-binding-dependent manner by diluting laminin ternary node complexes. The more Net 4 molecules are present, the softer the laminin network and the more resistant it is to metastases formation (Fig. 6a). We demonstrate that stiffness is a stronger influencing factor than pore size in the regulation of cancer cell movement through the BM. Thus, we propose a model where BM mechanics determine the ability of cancer cells to breach the BM: the softer a BM, the less breaching by cancer cells (Fig. 6b), extending patient survival.

Our experimental and theoretical data show that the stiffness of the laminin network can be precisely modulated by Net4, resulting in an altered stiffness of the entire BM and vessels. Our study reveals a critical role of $\mathrm{BM}$ mechanics at the primary and secondary tumour site independent of tumour-mediated alterations. Therefore, we hypothesize that the 'normal' BM composition may also play a pivotal role in the colonization of other common metastatic sites such as the liver, brain and bone (Fig. 6c) for all metastasizing solid tumours. Excitingly, our data suggest that baseline BM stiffness may predetermine survival even before having cancer throughout one's lifetime. Therefore, the development of tools 
to soften BMs at the primary and secondary site could represent a promising approach to decrease or even prevent metastasis.

A vast number of studies report pro- and antitumorigenic activity of Net4. However, all these studies have exclusively focused on a direct impact of Net 4 on cells ${ }^{28-37}$. Our study consistently revealed an indirect antimetastatic activity of Net4 when localized within native BMs, thereby modifying the mechanical properties of this specialized ECM material in many different cancer types. Thus, our data demonstrate the two context-dependent faces of Net4, such that Net 4 can be proinvasive via an unknown mechanism and antimetastatic via its laminin interaction which is strongly dependent on the ratio between Net 4 and laminin molecules.

The ECM is shaped by a huge number of extracellular proteins defined as the matrisome ${ }^{55}$. Previous reports have focused on the direct impact of single matrisome proteins on cells. However, our data open the possibility that some of these proteins might be reversible regulators of the biochemical and mechanical properties of the overall ECM structure simply through binding to ECM macromolecules. Consequently, our study might open a research area focusing on reversible extracellular matrix regulators (RevMatriRegs) of mechanical properties of the major network-forming proteins.

\section{Online content}

Any methods, additional references, Nature Research reporting summaries, source data, extended data, supplementary information, acknowledgements, peer review information; details of author contributions and competing interests; and statements of data and code availability are available at https://doi.org/10.1038/ s41563-020-00894-0.

Received: 17 January 2020; Accepted: 27 November 2020; Published online: 25 January 2021

\section{References}

1. Dillekas, H., Rogers, M. S. \& Straume, O. Are $90 \%$ of deaths from cancer caused by metastases? Cancer Med. 8, 5574-5576 (2019).

2. Chaffer, C. L. \& Weinberg, R. A. A perspective on cancer cell metastasis. Science 331, 1559-1564 (2011).

3. Fidler, I. J. The pathogenesis of cancer metastasis: the 'seed and soil' hypothesis revisited. Nat. Rev. Cancer 3, 453-458 (2003).

4. Valastyan, S. \& Weinberg, R. A. Tumor metastasis: molecular insights and evolving paradigms. Cell 147, 275-292 (2011).

5. Yurchenco, P. D. Basement membranes: cell scaffoldings and signaling platforms. Cold Spring Harb. Perspect. Biol. https://doi.org/10.1101/ cshperspect.a004911 (2011).

6. McKee, K. K., Harrison, D., Capizzi, S. \& Yurchenco, P. D. Role of laminin terminal globular domains in basement membrane assembly. J. Biol. Chem. 282, 21437-21447 (2007).

7. Abrams, G. A., Goodman, S. L., Nealey, P. F., Franco, M. \& Murphy, C. J. Nanoscale topography of the basement membrane underlying the corneal epithelium of the rhesus macaque. Cell Tissue Res. 299, 39-46 (2000).

8. Gaiko-Shcherbak, A. et al. The acinar cage: basement membranes determine molecule exchange and mechanical stability of human breast cell acini. PLoS ONE 10, e0145174 (2015).

9. Yurchenco, P. D. \& Ruben, G. C. Basement membrane structure in situ: evidence for lateral associations in the type IV collagen network. J. Cell Biol. 105, 2559-2568 (1987).

10. Linder, S. Invadosomes at a glance. J. Cell Sci. 122, 3009-3013 (2009).

11. Wisdom, K. M. et al. Matrix mechanical plasticity regulates cancer cell migration through confining microenvironments. Nat. Commun. 9, 4144 (2018).

12. Glentis, A. et al. Cancer-associated fibroblasts induce metalloprotease-independent cancer cell invasion of the basement membrane. Nat. Commun. 8, 924 (2017).

13. Couchman, J. R. \& Gibson, W. T. Expression of basement membrane components through morphological changes in the hair growth cycle. Dev. Biol. 108, 290-298 (1985).

14. Nelson, T. R., Charonis, A. S., McIvor, R. S. \& Butkowski, R. J. Identification of a cDNA encoding tubulointerstitial nephritis antigen. J. Biol. Chem. 270, 16265-16270 (1995).

15. Sasaki, T., Gohring, W., Pan, T. C., Chu, M. L. \& Timpl, R. Binding of mouse and human fibulin-2 to extracellular matrix ligands. J. Mol. Biol. 254, 892-899 (1995).
16. Sato, S. et al. Pikachurin, a dystroglycan ligand, is essential for photoreceptor ribbon synapse formation. Nat. Neurosci. 11, 923-931 (2008).

17. Veit, G. et al. Collagen XXVIII, a novel von Willebrand factor A domain-containing protein with many imperfections in the collagenous domain. J. Biol. Chem. 281, 3494-3504 (2006).

18. Timpl, R. \& Brown, J. C. Supramolecular assembly of basement membranes. Bioessays 18, 123-132 (1996).

19. Mayorca-Guiliani, A. E. et al. Decellularization and antibody staining of mouse tissues to map native extracellular matrix structures in 3D. Nat. Protoc. https://doi.org/10.1038/s41596-019-0225-8 (2019).

20. Koch, M. et al. A novel member of the netrin family, beta-netrin, shares homology with the beta chain of laminin: identification, expression, and functional characterization. J. Cell Biol. 151, 221-234 (2000).

21. van de Vijver, M. J. et al. A gene-expression signature as a predictor of survival in breast cancer. N. Engl. J. Med. 347, 1999-2009 (2002).

22. Nicolau, M., Tibshirani, R., Borresen-Dale, A. L. \& Jeffrey, S. S. Disease-specific genomic analysis: identifying the signature of pathologic biology. Bioinformatics 23, 957-965 (2007).

23. Li, Y. N. et al. The expression and function of netrin-4 in murine ocular tissues. Exp. Eye Res. 96, 24-35 (2012).

24. George, E. L., Georges-Labouesse, E. N., Patel-King, R. S., Rayburn, H. \& Hynes, R. O. Defects in mesoderm, neural tube and vascular development in mouse embryos lacking fibronectin. Development 119, 1079-1091 (1993).

25. Miner, J. H., Cunningham, J. \& Sanes, J. R. Roles for laminin in embryogenesis: exencephaly, syndactyly, and placentopathy in mice lacking the laminin alpha5 chain. J. Cell Biol. 143, 1713-1723 (1998).

26. Higgins, J. P. et al. Gene expression in the normal adult human kidney assessed by complementary DNA microarray. Mol. Biol. Cell 15, 649-656 (2004).

27. Zhao, $\mathrm{H}$. et al. Gene expression profiling predicts survival in conventional renal cell carcinoma. PLoS Med. 3, e13 (2006).

28. Hu, Y. et al. Netrin-4 promotes glioblastoma cell proliferation through integrin beta4 signaling. Neoplasia 14, 219-227 (2012).

29. Jayachandran, A. et al. Identifying and targeting determinants of melanoma cellular invasion. Oncotarget 7, 41186-41202 (2016).

30. Larrieu-Lahargue, F., Welm, A. L., Thomas, K. R. \& Li, D. Y. Netrin-4 induces lymphangiogenesis in vivo. Blood 115, 5418-5426 (2010).

31. Lv, B. et al. Netrin-4 as a biomarker promotes cell proliferation and invasion in gastric cancer. Oncotarget 6, 9794-9806 (2015).

32. Villanueva, A. A. et al. The netrin- $4 /$ neogenin- 1 axis promotes neuroblastoma cell survival and migration. Oncotarget 8 , 9767-9782 (2017).

33. Li, L. et al. NEtrin-4 protects glioblastoma cells from temozolomide induced senescence. PLoS ONE 8, e80363 (2013).

34. Esseghir, S. et al. Identification of NTN4, TRA1, and STC2 as prognostic markers in breast cancer in a screen for signal sequence encoding proteins. Clin. Cancer Res. 13, 3164-3173 (2007).

35. Eveno, C. et al. Netrin-4 delays colorectal cancer carcinomatosis by inhibiting tumor angiogenesis. Am. J. Pathol. 178, 1861-1869 (2011).

36. Fujikane, T. et al. Genomic screening for genes upregulated by demethylation revealed novel targets of epigenetic silencing in breast cancer. Breast Cancer Res. Treat. 122, 699-710 (2010).

37. Nacht, M. et al. Netrin-4 regulates angiogenic responses and tumor cell growth. Exp. Cell Res. 315, 784-794 (2009).

38. Kleinman, H. K. et al. Basement membrane complexes with biological activity. Biochemistry 25, 312-318 (1986).

39. Reuten, R. et al. Structural decoding of netrin-4 reveals a regulatory function towards mature basement membranes. Nat. Commun. 7, 13515 (2016).

40. Mayorca-Guiliani, A. E. et al. ISDoT: in situ decellularization of tissues for high-resolution imaging and proteomic analysis of native extracellular matrix. Nat. Med. 23, 890-898 (2017).

41. Trout, A. T., Rabinowitz, R. S., Platt, J. F. \& Elsayes, K. M. Melanoma metastases in the abdomen and pelvis: frequency and patterns of spread. World J. Radiol. 5, 25-32 (2013).

42. Tirosh, I. et al. Dissecting the multicellular ecosystem of metastatic melanoma by single-cell RNA-seq. Science 352, 189-196 (2016).

43. Cancer Genome Atlas, N. Genomic classification of cutaneous melanoma. Cell 161, 1681-1696 (2015).

44. Tucker, W. D., Arora, Y. \& Mahajan, K. Anatomy, Blood Vessels (StatPearls, 2020).

45. Candiello, J. et al. Biomechanical properties of native basement membranes. FEBS J. 274, 2897-2908 (2007)

46. Halfter, W. et al. Protein composition and biomechanical properties of in vivo-derived basement membranes. Cell Adh. Migr. 7, 64-71 (2013).

47. Last, J. A., Liliensiek, S. J., Nealey, P. F. \& Murphy, C. J. Determining the mechanical properties of human corneal basement membranes with atomic force microscopy. J. Struct. Biol. 167, 19-24 (2009). 
48. Bhave, G., Colon, S. \& Ferrell, N. The sulfilimine cross-link of collagen IV contributes to kidney tubular basement membrane stiffness. Am. J. Physiol. Ren. Physiol. 313, F596-F602 (2017).

49. Chlasta, J. et al. Variations in basement membrane mechanics are linked to epithelial morphogenesis. Development 144, 4350-4362 (2017).

50. Swift, J. et al. Nuclear lamin-A scales with tissue stiffness and enhances matrix-directed differentiation. Science 341, 1240104 (2013).

51. Irianto, J., Pfeifer, C. R., Xia, Y. \& Discher, D. E. SnapShot: mechanosensing matrix. Cell 165, 1820-1820 e1821 (2016).

52. Pearce, O. M. T. et al. Deconstruction of a metastatic tumor microenvironment reveals a common matrix response in human cancers. Cancer Discov. 8, 304-319 (2018)
53. Wullkopf, L. et al. Cancer cells' ability to mechanically adjust to extracellular matrix stiffness correlates with their invasive potential. Mol. Biol. Cell 29, 2378-2385 (2018).

54. Lubensky, T. C., Kane, C. L., Mao, X., Souslov, A. \& Sun, K. Phonons and elasticity in critically coordinated lattices. Rep. Prog. Phys. 78, 073901 (2015).

55. Naba, A. et al. The matrisome: in silico definition and in vivo characterization by proteomics of normal and tumor extracellular matrices. Mol. Cell. Proteomics 11, M111 014647 (2012).

Publisher's note Springer Nature remains neutral with regard to jurisdictional claims in published maps and institutional affiliations.

(c) The Author(s), under exclusive licence to Springer Nature Limited 2021 


\section{Methods}

Gene expression analysis. We used the $\mathrm{NKI}^{21}$ dataset of 295 breast cancers (https://ccb.nki.nl/data/) and a dataset with 13 normal breast tissue samples ${ }^{22}$, three from reduction mammoplasties and ten from pathologically normal breast tissue from breast cancer patients, to explore association with survival of all $\mathrm{BM}$ genes. We found 35 out of $49 \mathrm{BM}$ genes present in the NKI dataset. DSGA ${ }^{22}$ was used to decompose the data into Disease and Normal components. Roughly, the normal tissue breast samples are used to construct a mathematical model for the healthy state. This healthy-state model (HSM) incorporates a multitude of normal breast tissue signatures, including single-cell-type signatures as well as combinations of cell types in a range of states. Mathematically it is obtained from a linear model of the normal tissue data, followed by several steps of dimensionality reduction. The dimensionality reduction steps avoid overfitting the normal tissue data. Each tumour tissue gene expression sample is then mathematically decomposed into a Normal and a Disease component. The Normal component of the tumour is the signature of the cell types present in the tumour sample in their normal/healthy state. It is the profile of the mix of cells present in the tumour tissue sample, but with their healthy/normal gene expression signature. Mathematically it is the best fit of the tumour data to the HSM. The Disease component captures the modifications in the tumour sample from the normal signature; thus in essence the Disease component measures how the sample of cells, tumour and microenvironment have deviated from their normal/healthy phenotype. Mathematically, the Disease component is the vector of residuals from the HSM fit. It is important to point out that the DSGA decomposition of each tumour is done independently of the rest of the tumours in the cohort. In the next step, we first identified the status of each tumour based on the Disease component expression levels of ESR1 and ERBB2 (HER2). The distributions of both ESR1 and ERBB2 in the Disease component was clearly bimodal, and the cutoff was assigned between positive (over) and negative (under) expression status. We then explored the association with survival for each of the $35 \mathrm{BM}$ genes separately, in each of the following breast cancer subset of tumours: ER-negative tumours; ER-positive tumours; HER2 (ERBB2)-overexpressing tumours;

basal-like tumours (ER-negative, HER2-normal); luminal A tumours (ER-positive, HER2-normal); luminal B tumours (ER-positive, HER2-overexpressing); and HER2-overexpressing, ER-negative tumours. In the NKI dataset ER-negative, HER2-normal tumours are also PR-negative, thus basal-like. These subsets of tumours clearly overlap. The goal was to explore the association with survival in groups of tumours that were, to some degree, homogeneous with respect to the effects of the particular BM genes studied. For each of the $35 \mathrm{BM}$ genes, inside each of the seven subsets of breast cancer patients, we explored the association of the gene with survival:death and metastasis. We used Cox proportional-hazard models to compute the $P$ value of the separation in survival between the tumours with low levels of the gene (33rd percentile) and those with high levels of the gene (67th percentile). We also assigned a value of $+1,-1$ or 0 if the gene was associated with a good or bad prognosis, or neither. This was done for seven patient groups, 35 genes, Disease components and Normal components of data. Thus, for each BM gene we obtained a vector of $28 P$ values, and a vector of 28 prognosis signs: $+1,-1,0$. For each gene, we computed the log base 10 of the $P$ values vector. Our criteria are: (1) consistent association with survival across many tumour groups, and (2) highly significant association with survival. Thus, a consensus association is the mean of the signed prognosis vector $\pm \log _{10}(P): \frac{1}{28} \sum \pm \log _{10}(P)$, and an overall association is the sum of all the $\log$-transformed $P$ values $\sum \log _{10}(P)$. Thus, a large overall association value indicates strong association with survival, either in a few groups or across many groups. Large positive or small negative consensus association indicates association with survival across many different tumour groups. In case of the kidney analysis, gene expression from 166 renal cell carcinoma tumours (GSE3538) ${ }^{27}$ and 34 normal tissue samples (GSE3931) ${ }^{26}$ was analysed. DSGA analysis generated the decomposition into the Disease and Normal components of tumour data.

Mouse studies. All animal experiments followed the regulatory standards of, and were approved by, the Animal Experimentation Council of the Ministry of Environment and Food of Denmark. All experiments are controlled by the Danish Inspectorate for Animal Experimentation (permission number 2017-15-020101265) according to the Danish Law of Animal Welfare. All mice were housed in individually ventilated cages with a humidity of $55 \% \pm 10 \%$, a temperature of $22^{\circ} \mathrm{C} \pm 2{ }^{\circ} \mathrm{C}$, and a dark/light cycle of $12 \mathrm{~h} / 12 \mathrm{~h}$ with light from 6:00 to $18: 00$.

Cell culture. HEK293 (M. Koch, University Hospital Cologne, EBNA), mouse melanoma HCmel12 (T. Tüting, University Hospital Magdeburg, C57BL/6-compatible cell line) ${ }^{56}$, mouse breast cancer E0771 (R. Anderson, University of Melbourne, C57BL/6-compatible cell line) ${ }^{57}$, mouse breast cancer 4T1 (Karmanos Cancer Institute) ${ }^{58}$, mouse pancreatic cancer KPC (J. Morton, Beatson Institute) ${ }^{59}$ and human breast cancer MDA-MB-231 (J. Massagué, Memorial Sloan Kettering Cancer Center) ${ }^{60}$ cells were cultured in Dulbecco's modified Eagle medium (DMEM)/F-12, GlutaMAX supplement (DMEM/F-12, GlutaMAX supplement, Gibco) supplemented with $10 \%$ fetal bovine serum (FBS) and 20\% FBS in case of HCmel12 and E0771. Immortalized murine cancer-associated fibroblast cell lines (mCAF1 and mCAF2, E. Sahai, Francis
Crick Institute ${ }^{61}$ were cultured in DMEM/10\% FBS supplemented with insulin-transferrin-selenium.

Orthotopic mouse breast cancer model. Female mice at the age of 8 weeks from Net4 knockout and the corresponding wildtype litters were used. Cohorts of wildtype and Net4-deficient mice were orthotopically injected with $5 \times 10^{5} \mathrm{E} 0771$ cells into the mammary fat pad. Tumour growth was monitored on a regular basis and primary tumours were resected when they reached $1.5 \mathrm{~cm}$ in diameter. Mice were killed $28 \mathrm{~d}$ after primary tumour resection. The number of macroscopically visible metastases on lung surfaces were counted in a blind fashion. Lungs were further immunohistochemically (haemotoxylin \& eosin (H\&E)) processed to determine and analyse microscopic lung metastases using NanoZoomer Digital Pathology (NDP) scan (NDP.scan version 2.5.90) and view 2 (NDP.view version 2.7.52) software.

Expression and purification of recombinant Net4 proteins. Full-length Net4 from Mus musculus (Net4, NP_067295.2; amino acids, 20-628) and its respective laminin-binding mutant E195A,R199A (Mut) were cloned into a modified Sleeping-beauty vector ${ }^{62}$ with a double N-terminal Strep II tag. A pool of HEK293 cells was stably transfected followed by screening for high-level expression. Net4 and its laminin-binding mutant version were purified by Strep-Tactin Sepharose (IBA). The purified proteins were dialysed against $1 \times$ PBS. These proteins were further sterile filtered through a $0.22 \mu \mathrm{m}$ filter for all in vitro experiments.

Proliferation assay. Cancer cells were plated at 2,000 cells per 96 wells and treated without or with different concentrations $\left(0.1,3.6\right.$ and $\left.5.2 \mu \mathrm{g} \mathrm{well}^{-1}\right)$ of recombinant Net4 for $96 \mathrm{~h}$. Afterwards cells were washed twice with $100 \mu \mathrm{l}$ of $1 \times$ PBS and $100 \mu \mathrm{l}$ DMEM/F-12, GlutaMAX supplement was added to the cells and the plate was incubated at $37^{\circ} \mathrm{C}$ for $1 \mathrm{~h}$. After $1 \mathrm{~h}, 20 \mu \mathrm{l}$ of MTS solution (Promega) was added to each well including blank wells. Absorbance was measured at $492 \mathrm{~nm}$ using a SpectraMax Paradigm plate reader (Molecular Devices; SoftMax Pro version 6.5.1 software) after $1 \mathrm{~h}$. Blank values were subtracted from each value and values were normalized to the respective control wells.

Contraction assay. Cancer-associated fibroblasts (mCAF1 and mCAF2) were mixed with $2 \mathrm{mg} \mathrm{ml}^{-1}$ collagen I matrix, and $100 \mu \mathrm{l}$ of cell/collagen I mix was plated per well in a 96 well plate to give 80,000 cells well $^{-1}$. Afterwards, $200 \mu$ of media containing $2 \%$ FBS supplemented without (control) and with different amounts of recombinant Net 4 and its respective laminin-binding mutant Net4 E195A,R199A. Cells were allowed to contract the collagen I matrix for $72 \mathrm{~h}$. Afterwards, the contracted area was analysed and normalized to the control of the respective repeat.

\section{Immunohistochemistry and immunofluorescence staining of E0771 primary} tumours. Mouse tumours were resected, fixed in $10 \%$ neutral buffered formalin at $4^{\circ} \mathrm{C}$ overnight, and paraffin embedded according to a standard protocol. For immunohistochemistry staining, tissue sections were deparaffinized in xylene and rehydrated in graded ethanol $(100 \%, 95 \%, 70 \%)$. Antigen retrieval was performed for $15 \mathrm{~min}$ at $95-98^{\circ} \mathrm{C}$ in $10 \mathrm{mM}$ sodium citrate buffer, $\mathrm{pH}$ 6.0. Then, endogenous peroxidase activity was quenched by incubation in $3 \% \mathrm{H}_{2} \mathrm{O}_{2}$ for $10 \mathrm{~min}$. Sections were blocked in PBS $+0.1 \%$ Tween-20 and 5\% goat or donkey serum. Incubations with primary antibodies (CD8a; Cell Signaling, 98941, clone D4W2Z; 1:200) were performed overnight at $4^{\circ} \mathrm{C}$ in a humidified chamber, followed by appropriate horseradish peroxidase-conjugated secondary antibodies (Dako, K4001 or K4003) at room temperature. ImmPACT DAB Kit (Vector Laboratories, SK-4105) was used to develop signals according to the manufacturer's instructions. Sections were counterstained with haematoxylin and mounted using DPX mounting medium (CellPath, SEA-1304-00A).

For immunofluorescence staining of tumour slides, antigen retrieval was performed similarly to immunohistochemistry staining, except slides were not incubated with $3 \% \mathrm{H}_{2} \mathrm{O}_{2}$. Primary antibodies ( $\alpha \mathrm{SMA}$; Abcam, ab5694; 1:200 and F4/80; eBioscience, 14-4801-85, Clone BM8; 1:100) were incubated at $4{ }^{\circ} \mathrm{C}$ overnight in a humidified chamber followed by incubation with Alexa Fluor-conjugated secondary antibodies including DAPI $\left(1 \mu \mathrm{g} \mathrm{ml}^{-1}\right)$ at room temperature for $1 \mathrm{~h}$ and mounted using Dako Fluorescent Mounting Medium. Stained sections were imaged using an SP8 confocal microscope (Leica) with Leica Application Suite X software (version 3.5.5.19976).

Preparation of Net4-containing Matrigel matrix. All experiments in which Matrigel has been supplemented with full-length Net4 have been performed at a laminin 111 to Net4 molecular ratio of 2:1. Matrigel containing 50\% Net4 molecules compared with laminin 111 molecules was prepared by taking the protein concentration of Matrigel, which was between 8 and $11 \mathrm{mg} \mathrm{ml}^{-1}$ depending on the batch. Afterwards, the estimated laminin 111 content of Matrigel is $60 \%$. Moreover, the molecular weight of laminin 111 is around 10 -fold higher than the molecular weight of Net4. As an example, $1 \mathrm{ml}$ of a $10 \mathrm{mg} \mathrm{ml}^{-1}$ Matrigel matrix mix was supplemented with $300 \mu \mathrm{g}$ full-length Net4 to generate a Matrigel matrix containing approximately $50 \%$ Net 4 molecules compared with laminin 111 molecules. The amount of recombinant Net 4 within one $20 \mu$ matrix 
sphere was approximately 5.2 and $2.6 \mu \mathrm{g}$ for $50 \%$ and $25 \%$ Net 4 , respectively, in proportion to laminin.

Sphere invasion assay. MDA-MB-231 and HCmel12 cells were adjusted to 100,000 cells $\mathrm{ml}^{-1}$ in DMEM/F-12, GlutaMAX supplement with $10 \%$ FBS, $0.24 \%$ methylcellulose and $32 \mu \mathrm{g}$ collagen I. Liquid drops of $20 \mu \mathrm{l}$ of this mix were placed on the lid of a $10 \mathrm{~cm}$ cell culture dish. The lid was placed on top of a dish containing $6 \mathrm{ml}$ of $1 \times \mathrm{PBS}$ and cells were allowed to form spheres in the hanging drops for $120 \mathrm{~h}$. Afterwards, formed spheres were embedded in $20 \mu \mathrm{l}$ Matrigel and in $20 \mu \mathrm{l} \mathrm{Net4-binding} \mathrm{or} \mathrm{laminin-binding} \mathrm{mutant-containing} \mathrm{Matrigel,} \mathrm{each}$ supplemented with $0.5 \mathrm{mg} \mathrm{ml}^{-1}$ collagen I. In case of the collagen I sphere invasion experiments, formed spheres were embedded in $20 \mu \mathrm{l} 1.5 \mathrm{mg} \mathrm{ml}^{-1}$ collagen I matrix and in $20 \mu \mathrm{l}$ Net4- or laminin-binding mutant-containing collagen I matrix. Plates containing the plugs with spheres were placed upside down at $37^{\circ} \mathrm{C}$ for $1 \mathrm{~h}$ to allow Matrigel polymerization. Afterwards, plates were filled with DMEM/F-12, GlutaMAX. Sphere invasion was imaged using a bright-field microscope (Olympus CKX53 with Olympus DP22 camera and Olympus cellSens Entry software version 1.16) after 18 and $24 \mathrm{~h}$ for HCmel12 and MDA-MB-231 cells, respectively. Invasive cell area was quantified using ImageJ version $2.1 .0 / 1.53 \mathrm{c}$.

\section{Decellularization and staining of mouse lung and mesentery tissues. Net4} wildtype and knockout mice were killed and operated on as described previously ${ }^{19}$ to perfuse through the aorta and the superior mesenteric artery for lungs and mesentery, respectively. Catheterized organs are perfused by a system in which a peristaltic pump (Ole Dich) connects a reagent reservoir to the catheter connector with sterile silicone tubes (Ole Dich). The tubing has a lumen diameter of $2 \mathrm{~mm}$ and an external diameter of $4 \mathrm{~mm}$. The waste-collection tubing has a lumen of $2.5 \mathrm{~mm}$ and an external diameter of $4.5 \mathrm{~mm}$. The peristaltic pump is set to a flow output of $0.2 \mathrm{ml} \mathrm{min}^{-1}$. Perfusion begins with $15 \mathrm{~min}$ of deionized water. This is followed by a detergent regimen of $0.5 \%$ sodium deoxycholate in deionized water for $24 \mathrm{~h}$. Decellularized lung and mesentery tissues were stored in deionized water at $4{ }^{\circ} \mathrm{C}$ until staining was initiated. After decellularization, lung and mesentery tissues were blocked with $6 \%$ donkey serum and $3 \%$ bovine serum albumin in $1 \times$ PBS overnight. On the next day, the block solution was discarded and primary antibodies for Net4 (polyclonal rabbit anti-Net4; 1:100), laminin $\gamma 1$ (monoclonal rat anti-laminin $\gamma 1$, Millipore MAB1914P; 1:100) and collagen IV (polyclonal goat anti-collagen IV, Millipore AB769; 1:100) in 3\% donkey serum in $1 \times$ PBS were added to the lungs and incubated overnight. Afterwards, the primary antibody solution was discarded and lung as well as mesentery tissues were washed with $0.05 \%$ Tween-20 in $1 \times$ PBS at least six times for $1 \mathrm{~h}$. Then the secondary antibodies anti-rabbit Alexa488, anti-rat Alexa555 and anti-goat Alexa647 (1:1,000) in 3\% donkey serum in $1 \times$ PBS were added overnight. On the next day, the tissue was washed with $0.05 \%$ Tween-20 in $1 \times$ PBS three times for $1 \mathrm{~h}$ and subsequently imaged with an inverted Leica SP5-X MP 2-photon confocal microscope connected to a Ti-sapphire laser (Spectra Physics MaiTai HP DeepSee Laser, Spectral Physics; tunable wavelength $690-1,040 \mathrm{~nm}$ ) and a Supercontinuum white light laser with the Leica Application Suite X software version 2.7.4.10100.

Sample preparation for mass spectrometry. Lysate preparation and digestion was done according to Kulak et al. ${ }^{63}$ with some modifications. Briefly, five lung tissue samples from both Net4 wildtype and knockout mice were lysed using $30 \mu \mathrm{l}$ of lysis buffer (consisting of $6 \mathrm{M}$ guanidinium hydrochloride, $10 \mathrm{mM}$ tris(2-carboxyethyl)phosphine, $40 \mathrm{mM}$ chloroacetamide, $50 \mathrm{mM}$ HEPES $\mathrm{pH}$ 8.5 ) in a Barocycler 2320EXT (Pressure BioSciences) set to 30 cycles of 45,000 p.s.i., $50 \mathrm{~s}$ on, $10 \mathrm{~s}$ off. Samples were boiled at $95^{\circ} \mathrm{C}$ for $5 \mathrm{~min}$, after which they were sonicated on the 'high' setting for $5 \times 30 \mathrm{~s}$ in a Bioruptor sonication water bath (Diagenode) at $4^{\circ} \mathrm{C}$. After determining protein concentration with a Pierce Gold BCA kit (Thermo Fisher Scientific), $20 \mu \mathrm{g}$ was taken forward for digestion. Samples were diluted 1:3 with 10\% acetonitrile, $50 \mathrm{mM}$ HEPES, pH 8.5, LysC (mass spectrometry grade, Wako) was added in a 1:50 (enzyme to protein) ratio, and samples were incubated at $37^{\circ} \mathrm{C}$ for $4 \mathrm{~h}$. Samples were further diluted to $1: 10$ with $10 \%$ acetonitrile, $50 \mathrm{mM}$ HEPES pH 8.5 , trypsin (mass spectrometry grade, Promega) was added in a 1:100 (enzyme to protein) ratio, and samples were incubated overnight at $37^{\circ} \mathrm{C}$. Enzyme activity was quenched by adding $2 \%$ trifluoroacetic acid (TFA) to a final concentration of $1 \%$. Prior to TMT labelling, the peptides were desalted on a SOLA $\mu$ C18 plate (Thermo Fisher Scientific). For each sample, the C18 material was activated with $200 \mu$ of $100 \%$ methanol (HPLC grade, Sigma), and $200 \mu \mathrm{l}$ of buffer B ( $80 \%$ acetonitrile, $0.1 \%$ formic acid). The C18 bedding was subsequently equilibrated twice with $200 \mu$ of buffer A $(0.1 \%$ formic acid), after which $20 \mu \mathrm{g}$ of sample was loaded using centrifugation at 1,000 r.p.m. After washing the $\mathrm{C} 18$ bedding twice with $200 \mu \mathrm{l}$ of buffer $\mathrm{A}$, the peptides were eluted into clean $1.5 \mathrm{ml}$ Eppendorf tubes using $40 \%$ acetonitrile and $0.1 \%$ formic acid. The eluted peptides were concentrated in an Eppendorf Speedvac and reconstituted in $100 \mathrm{mM}$ tetraethylammonium bromide ( $\mathrm{pH} 8.5$ ) for TMT labelling. Labelling was done according to the manufacturer's instructions, and labelled peptides were subsequently mixed 1:1:1:1:1:1:1:1:1:1:1:1:1:1:1:1 (16-plex), acidified to $1 \%$ TFA and the acetonitrile concentration brought down to $<5 \%$ using $2 \%$ TFA. Prior to mass spectrometry analysis, the peptides were fractionated using an offline Thermo Fisher Ultimate 3000 liquid chromatography system using high $\mathrm{pH}$ fractionation ( $5 \mathrm{mM}$ ammonium bicarbonate, $\mathrm{pH} 10)$ at a flowrate of $5 \mu \mathrm{lmin}{ }^{-1}$. Peptides $(20 \mu \mathrm{g}$ ) were separated over a $120 \mathrm{~min}$ gradient (from $5 \%$ to $35 \%$ acetonitrile), while collecting fractions every $120 \mathrm{~s}$. The resulting 60 fractions were pooled into 30 final fractions (fractions $1+31,2+32,3+33$, and so on), acidified to $\mathrm{pH}<2$ with $1 \%$ TFA and loaded onto EvoSep StageTips according to the manufacturer's protocol.

\section{Global TMT-labelled liquid chromatography-mass spectrometry data} acquisition. For each fraction, peptides were analysed using the preset ' 30 samples per day' method on the EvoSep One instrument. Peptides were eluted over a 44 min gradient and analysed on an Exploris 480 instrument (Thermo Fisher Scientific) running in a data-dependent tandem mass spectrometry top-speed mode ( $3 \mathrm{~s}$ cycle time). Full mass spectrometry spectra were collected at a resolution of 60,000 , with a normalized automatic gain control target of $300 \%$ and automatic maximum injection time, with a scan range of $375-1,500 \mathrm{~m} / z$. The tandem mass spectrometry spectra were obtained at a resolution of 30,000 with the 'turboTMT' functionality enabled, with a normalized automatic gain control target of $100 \%$ and automatic maximum injection time, a normalized collision energy of 33 and an intensity threshold of $8 e^{3}$. First mass was set to $120 \mathrm{~m} / z$ to ensure capture of the TMT reporter ions, and precursors were isolated with a $0.7 \mathrm{~m} / z$ isolation window and Precursor Fit enabled to $70 \%$ at a window of $0.7 \mathrm{~m} / z$. Dynamic exclusion was set to $60 \mathrm{~s}$, and ions with a charge state $<2,>7$ and unknown were excluded. Mass spectrometry performance was verified for consistency by running complex cell lysate quality-control standards, and chromatography was monitored to check for reproducibility.

TMT quantitative proteomics analysis. The raw files were deposited in the PRIDE database of ProteomeXchange (PXD022145) and analysed using Proteome Discoverer 2.4. TMTPro reporter ion quantitation was enabled in the processing and consensus steps, and spectra were matched against the Mus musculus protein database obtained from Uniprot. Dynamic modifications were set as oxidation (M), deamidation (N,Q) and acetyl on protein $\mathrm{N}$ termini. Cysteine carbamidomethyl (on $\mathrm{C}$ residues) and TMTPro (on peptide $\mathrm{N}$ termini and $\mathrm{K}$ residues) were set as static modifications. All results were filtered to a $1 \%$ false discovery rate, and TMT reporter ion quantitation done using the built-in 'reporter ions quantifier' node, with statistical significance testing done with the built-in analysis of variance (ANOVA) test. Proteins were filtered for those with at least two peptides and visualized in R using the GGplot2 package or with GraphPad Prism.

Metastases formation. All animal experiments were conducted with 8-week-old mice; male as well as female animals from Net4 knockout and the corresponding wildtype litters were used. Cohorts of wildtype and Net4-deficient mice were intravenously injected with $5 \times 10^{5} \mathrm{HCmel} 12$ melanoma or $2 \times 10^{5}$ E0771 breast cancer cells. Mice were killed 20 and 28 days after injection of HCmel12 and E0771, respectively. The number of macroscopic metastases on lung surfaces and inside the abdomen (HCmel12) were counted in a blind fashion. In terms of the mouse breast cancer cell injection, lungs were further immunohistochemically (H\&E) processed to determine and analyse microscopic lung metastases using NDP software (NDP.scan version 2.5.90 and NDP.view version 2.7.52). The experimental setup for the rescue experiment was slightly modified. Here Net4 knockout mice were treated with $1 \times$ PBS (control) or $2.5 \mathrm{mg}$ per $\mathrm{kg}$ body weight recombinant Net4 for five consecutive days (days 1-5) prior to intravenous cell injection. After $5 \mathrm{~d}$ of treatment, animals were kept without any further treatment for an additional $2 \mathrm{~d}$ (days 6-7). On the following day (day 8), $5 \times 10^{5} \mathrm{HCmel} 12$ melanoma cells were injected intravenously into Net4 knockout mice treated with PBS or Net4. Mice were killed on day 28 ( $20 \mathrm{~d}$ after HCmel12 intravenous injection). The number of macroscopic metastases on lung surfaces were counted in a blind fashion.

Pressure myography. All animal work was performed in accordance with UK Home Office Regulations, covered by UK Animal Project license 70/8604 and approved by the University of Glasgow animal ethics committee. Myography was performed on 5-month-old Net4 knockout mice with wildtype littermates as controls. Animals were killed by cervical dislocation and third-order mesenteric arteries were collected by dissection from the mesentery within $1 \mathrm{~h}$ of killing using a dissecting microscope (Leica) in cooled calcium-free physiological salt solution (cf-PSS: $0.25 \mathrm{M} \mathrm{NaCl}, 0.001 \mathrm{M} \mathrm{KCl}, 2 \mathrm{mM} \mathrm{MgSO}_{4}, 50 \mathrm{mM} \mathrm{NaHCO}_{3}, 2 \mathrm{mM} \mathrm{KH}_{2} \mathrm{PO}_{4}$, $1 \mathrm{mM}$ glucose, $0.5 \mathrm{ml}$ of $23 \mathrm{mM}$ EDTA). Perivascular adipose tissue was removed, and mesenteric arteries were transferred into separate $1.5 \mathrm{ml}$ microcentrifuge tubes containing cf-PSS. Samples were kept on ice for pressure myography and used on the day of collection. Mesenteric artery rings were mounted on two glass cannulas on a pressure myograph (Danish Myo Technology) in cf-PSS and maintained at $37^{\circ} \mathrm{C}$ under $95 \% \mathrm{O}_{2} / 5 \% \mathrm{CO}_{2}$. The vessels were unbuckled and pressurized at $70 \mathrm{mmHg}$ for $30 \mathrm{~min}$ for equilibration. Vessels were then subjected to a pressure curve $(10,20,40,60,80,100,110$ and $120 \mathrm{mmHg}$ under passive conditions) using cf-PSS and measurements of internal $\left(D_{\mathrm{i}}\right)$ and external diameter $\left(D_{\mathrm{e}}\right)$ were collected at the end of $5 \mathrm{~min}$ at each pressure. Wall thickness, WT, was calculated as $\left(D_{\mathrm{e}}-D_{\mathrm{i}}\right) / 2$. Circumferential wall stress $\sigma$ was calculated as $\left(P \times D_{\mathrm{i}}\right) /(2 \times \mathrm{WT})$ where $P$ is the pressure. Circumferential wall strain $\varepsilon$ was calculated as $\left(D_{\mathrm{i}}-D_{\mathrm{i} @ 10 \mathrm{mmHg}}\right) /$ 
$\left(D_{\text {i@10 mmHg }}\right)$. Arterial stiffness independent of geometry is determined by Young's elastic modulus, $E$ (stress/strain ${ }^{64}$. Statistical analysis was performed with GraphPad Prism version 8.1.1 software calculating the linear regression of stressstrain curves for both Net 4 wildtype and knockout.

Quantitative rtPCR. HEK293, MDA-MB-231, E0771, 4T1 and HCmel12 cells were cultured in their respective media until a cell density of approximately $90 \%$ was reached. Cells were then trypsinized and centrifuged (1,000 r.p.m.; $5 \mathrm{~min}$ ). Cell pellets were frozen on liquid nitrogen and stored on $-80^{\circ} \mathrm{C}$ until further processing started. RNA was extracted using the RNeasy Mini Kit (Qiagen) following the manufacturer's instructions. After RNA extraction, RNA concentration was measured using a NanoDrop. Reverse transcription of $1 \mu \mathrm{g}$ of RNA per cell line and replicate was performed using Transcriptor Universal cDNA Master kit (Roche) following the manufacturer's instructions. Real-time primers corresponding to mouse Net4 (forward: TGAGAATGCTGACCTCACTTGC, reverse: GGCAGCGTTGCATTTATCACAC), mouse ribosomal protein lateral stalk subunit P0 (RPLP0) (forward: CATCATCAATGGGTACAAGCGC, reverse: CAGTAAGTGGGAAGGTGTACTC), human Net4

(forward: CCTGAACGAAGAGCCTCAACAT, reverse: GGTCTGAAGCCATGAACAGGTA) and human RPLP0 (forward: CTGCTGCCTCATATCCGGG, reverse: TGATCTCAGTGAGGTCCTCCTT) were validated for further analysis. Quantitative rtPCR was performed on a LightCycler 480 II (Roche; Light Cycler 480 SW version 1.5.1) by generating standard curves for mouse Net4 using HEK293 cells overexpressing mouse Net4, for human Net4 using HEK293 cells overexpressing human Net4, for the housekeeping gene mouse RPLP0 using E0771 cDNA and for the housekeeping gene human RPLP0 using HEK293 cDNA. Afterwards analysis of the standard curves using the Light Cycler 480 SW version 1.5.1 software was performed for each primer pair, resulting in the concentration of the transcript for each gene. Finally, the expression levels of mouse and human Net 4 transcripts were calculated relative to the concentration of the respective RPLP0 expression.

CRISPR/Cas9-mediated Ntn4 knockout in E0771 mouse breast cancer cells. The guide RNA (sgRNA) against the mouse Net4 gene (Ntn4) was generated using the sgRNA design platform of the Broad Institute (https://portals.broadinstitute. org/gpp/public/analysis-tools/sgrna-design). The sgRNA oligos were annealed and cloned into the PX458 CRISPR/Cas9 vector. After sequencing, the vector was transfected into E0771 mouse breast cancer cells. Transfected cells were single-cell sorted into 96 well plates via detection of green fluorescent protein using a fluorescence-activated cell sorter after $24 \mathrm{~h}$. Afterwards, cells were validated for Ntn 4 knockout via isolation of the gDNA followed by the amplification of the genomic region corresponding to the sgRNA binding region and cloned into a TOPO vector. Here, at least 10 clones were sequenced to identify specific Ntn4 knockout on all alleles. E0771 Ntn4-knockout cells were used for intravenous injection into Net4 knockout and the corresponding wildtype litter animals.

scRNA-seq analysis. Using scRNA-seq data in melanoma (GSE72056) ${ }^{42}$, which contains data of 4,645 cells from 19 melanoma patients, including tumours from ten lymphoid metastases, eight distant sites and primary melanoma, we scrutinized which cell types express NTN4 and additional BM-encoding genes (Supplementary Table 1). Based on cell-type-specific marker genes, we obtained cell clusters composed of seven cell types (Extended Data Fig. 6a): B cells, CAFs, endothelial cells, macrophages, melanoma cells, natural killer (NK) cells and T cells. We also investigated the expression of the additional 48 BM genes for each cell type (Extended Data Fig. 7). To find out the overall expression levels of the whole 49 $\mathrm{BM}$ genes in the seven cell types, we calculated an aggregate expression of the 49 $\mathrm{BM}$ genes using $z$ scores.

Atomic force microscopy. AFM indentation experiments on Matrigel and lung tissue sections were performed with a NanoWizard 1 (JPK Instruments with JPK Control software 5.0.130) equipped with a $100 \mu \mathrm{m} \times 100 \mu \mathrm{m} \times 15 \mu \mathrm{m}$ piezo scanner. The AFM was mounted on an inverted optical microscope (Axiovert 200, Carl Zeiss) placed on an active isolation table (Micro 60, Halcyonics). The whole setup was placed inside a custom-built $1 \mathrm{~m}^{3}$ soundproof box to reduce the influence of ambient noise. All AFM experiments were carried out in PBS at room temperature, using silicon nitride MLCT cantilevers (Bruker) with a nominal spring constant of $\sim 0.1 \mathrm{~N} \mathrm{~m}^{-1}$ for lung tissue sections and $\sim 0.03 \mathrm{~N} \mathrm{~m}^{-1}$ for Matrigel experiments. The cantilever force constant was determined for each cantilever individually, using the thermal noise method ${ }^{65}$. For all experiments, the Young's modulus was derived from the first $500 \mathrm{~nm}$ of the indentation part of the curves using a modified Hertz model $^{6,67}$. For data analysis, the JPK software (version 6.0.69) was used. The indentation experiments on the BM were performed on $15 \mu \mathrm{m}$ cryosections (Leica CM1950) of fresh, snap frozen, non-fixed lung tissue embedded in Tissue Tek OCT. The tissue slides were thawed and immersed in PBS for AFM measurements. First, overview force maps of $25 \mu \mathrm{m} \times 25 \mu \mathrm{m}$ were recorded on the alveolar wall showing the two cell layers and the BM. Then, three $1.5 \mu \mathrm{m} \times 1.5 \mu \mathrm{m}$ force maps, comprising the $\mathrm{BM}$ and the adjacent tissue were recorded. Each map consisted of 2,500 $(50 \times 50)$ indentation-retract cycles, rendering a lateral resolution of $30 \mathrm{~nm}$ for the subsequent Young's modulus analysis. Here, the $z$-piezo velocity was $10 \mu \mathrm{m} \mathrm{s}^{-1}$. Two alveolar walls were assessed for each animal. Because of time constraints, in these experiments, the cantilever force constant was assessed after recording the indentation maps. The cantilever deflection signal was limited to $1 \mathrm{~V}$.

In the mixing approach $100 \mu$ of Matrigel matrix (Corning, lot 9091003 , reference 354234 ) was gently mixed with $0.6 \%, 1 \%, 1.2 \%, 3 \%, 6 \%, 10 \%, 25 \%, 30 \%$ and $50 \%$ Net4 in PBS (Biochrom Dulbecco's PBS without $\mathrm{Mg}^{2+} / \mathrm{Ca}^{2+}, \mathrm{pH} 7.4$ ) on ice and pipetted into a silicon ring with an inner diameter of $1 \mathrm{~cm}$ on top of a precooled microscope slide with a precooled pipette and incubated for $30 \mathrm{~min}$ at $37^{\circ} \mathrm{C}$. The Matrigel sample was then transferred to the AFM stage, where a droplet of PBS was pipetted on top of the gel prior to AFM experiments. Maps of $10 \times 10$ force-distance curves were recorded with a $100 \mu \mathrm{m} \times 100 \mu \mathrm{m}$ scan area, at a $z$-piezo velocity of $5 \mu \mathrm{m} \mathrm{s}^{-1}$. The indentation force was limited to $2 \mathrm{nN}$. At least five force maps on at least two different gels with a total number of 373-939 force curves per map were analysed.

In the titration experiment $2 \mathrm{ml}$ of Matrigel matrix was plated on a precooled Petri dish $\left(-20^{\circ} \mathrm{C}\right)$ followed by an incubation time of $30 \mathrm{~min}$ at $37^{\circ} \mathrm{C}$. For AFM experiments $9 \mathrm{ml}$ of PBS was added and force curves were recorded on the pure Matrigel. Then $0.1 \%, 0.5 \%, 1 \%, 2.5 \%, 5 \%, 10 \%, 25 \%$ and $50 \%$ Net 4 were added in a titration procedure. Arrays of $10 \times 10$ force-distance curves were recorded in a $10 \mu \mathrm{m} \times 10 \mu \mathrm{m}$ scan area, at a $z$-piezo velocity of $5 \mu \mathrm{m} \mathrm{s}^{-1}$. In the titration experiments, the indentation force was limited to $3 \mathrm{nN}$. In case of the collagenase treatment, Matrigel without collagenase and Matrigel with $200 \mathrm{U}$ of collagenase (Worthington Biochemical) were allowed to polymerize at $37^{\circ} \mathrm{C}$ for $3 \mathrm{~h}$ before Net 4 titration $(1 \%, 2 \%, 6 \%$ and $10 \%)$ and constant AFM measurement.

Electron microscopy. Ketanest anaesthetized mice were perfusion fixed with $4 \%$ paraformaldehyde, $0.1 \mathrm{M} \mathrm{PBS}$ at $\mathrm{pH} 7.4$ by cannulation of the left ventricle for $15 \mathrm{~min}$. Following removal, the isolated lung tissue from perfusion-fixed mice was postfixed in $2 \%$ paraformaldehyde, $2 \%$ glutaraldehyde, $0.1 \mathrm{M}$ cacodylate buffer at $\mathrm{pH} 7.35$ for $6 \mathrm{~h}$. The lung tissue was rinsed in cacodylate buffer three times and then treated with $1 \%$ uranyl acetate in $70 \%$ ethanol for $8 \mathrm{~h}$ to enhance the contrast. The lung tissue was subsequently dehydrated in a graded series of ethanol and then embedded in Araldite (Serva). Semithin sections $(0.5 \mu \mathrm{m})$ were cut with a glass knife on an ultramicrotome (Reichert) and stained with methylene blue. Ultrathin sections $(70 \mathrm{~nm})$ for electron microscopic observation were processed on the same microtome with a diamond knife and placed on copper grids. Transmission electron microscopy was performed using an EM109 electron microscope (Zeiss) with ImageSP version 1.2.6.11 software.

Theoretical modelling. In the simulations, the laminin 111 network in the BM is modelled as an elastic honeycomb network representing the laminin molecules and an additional constant elastic background contribution representing the underlying collagen IV network. In a 2D simulation box with periodic boundary conditions, 512 nodes are arranged on a honeycomb lattice and connected by harmonic bonds with a finite equilibrium length, representing the $\alpha, \beta$ and $\gamma$ chains of the laminin heterotrimer. Dilution by Net 4 is realized by identifying binding sites in between neighbouring laminin heterotrimers and randomly removing a fraction of these sites according to the Net 4 concentration. This procedure is mathematically equivalent to site percolation on half of the sites of a honeycomb lattice. To obtain the elastic modulus, a longitudinal strain is applied by uniformly stretching the network stepwise in all fundamental lattice directions. After each stretching step, the energy of the elastic network is minimized with respect to the node positions using the conjugated gradient algorithm to obtain equilibrium positions and stretching energies. To these energy-strain curves, a purely quadratic energy function is added to account for the underlying collagen IV network. We averaged over 200 different dilution patterns per dilution fraction $\phi=\frac{\text { no.of removed sites }}{\text { no.of total sites }}$ to take care of effects arising from the random nature of dilution. We define the strain inflicted on the simulation box of area $A$ as $\epsilon=\frac{A^{\prime}-A}{A}$, where $A^{\prime}$ denotes the area of the stretched box. We obtain from the simulation the dilution-dependent energy density $w_{\phi}(\epsilon)$ as a function of the strain $\epsilon$. The non-linear relationship between the energy density $w_{\phi}(\epsilon)$ and the uniaxial strain $\epsilon$ of a diluted 2D honeycomb network has the functional form

$$
w_{\phi}(\epsilon)=\frac{1}{2} E_{\mathrm{col}} \epsilon^{2}+\frac{1}{2} E_{\mathrm{lam}}(\phi)\left(\epsilon-\epsilon^{*}(\phi)\right)^{2} \theta\left(\epsilon-\epsilon^{*}(\phi)\right),
$$

where $\theta(x)$ is the step function and $\epsilon^{*}(\phi)$ is the dilution-dependent threshold strain (Extended Data Fig. 10f). The modulus $E_{\text {lam }}(\phi)$ is extracted from 2D network simulations (Extended Data Fig. 10g) as the slope of the linear part of the stressstrain curve (Fig. 5e).

In our AFM experiments, the effective BM elastic modulus $\kappa(\phi)$ was determined by measuring force-indentation curves $F(\delta)$, where $\delta$ denotes the indentation depth. The stress and strain fields caused by a conical indenter in a $3 \mathrm{D}$ material are concentrated in a region near the surface of the material and in the immediate vicinity of the indenter ${ }^{68,69}$. We therefore approximate the extensional strain as the increase in surface area relative to the undeformed surface and calculate numerically the total energy of the deformed surface.

We estimate the extensional strain of an isotropic elastic medium at the surface indented by a cone by calculating the increased area of the deformed surface with respect to the undeformed state. 
In the case of an isotropic elastic medium indented by a cone at the surface, the shape of the deformed surface reads ${ }^{68}$

$$
u_{z}(r, \delta)= \begin{cases}\frac{2 \delta}{\pi}\left(\frac{r}{r_{1}}-\frac{\pi}{2}\right), & r \leq r_{1} \\ \frac{2 \delta}{\pi}\left(\frac{r}{r_{1}}-\sqrt{\left(\frac{r}{r_{1}}\right)^{2}-1}-\sin ^{-1}\left(\frac{r_{1}}{r}\right)\right), & r>r_{1}\end{cases}
$$

for an indentation depth of $\delta$ and an indentation-dependent contact radius $r_{1}(\delta)=\frac{2 \delta}{\pi} \tan (\alpha)$, where $\alpha$ denotes the half-opening angle of the cone. For our estimates, we choose half-opening angles of $\alpha=20^{\circ}, 40^{\circ}$ and $60^{\circ}$. Although a value of $\alpha=20^{\circ}$ corresponds best to the shape of the pyramidal tip used in the AFM experiments (Atomic force microscopy), presumably higher values of $\alpha$ are closer to reality with respect to phenomena neglected in our simple model, for example, imperfections of the AFM tip and the samples, torsional and radial strains as well as strains inflicted on the 3D material below the surface. The definitions of $\delta, r_{1}$ and $\alpha$ are sketched in Fig. 5f. Since the total area of the deformed surface is given by $S=2 \pi \int_{0}^{R} r \sqrt{1+\left(\frac{\mathrm{d} u_{z}}{\mathrm{~d} r}\right)^{2}} \mathrm{~d} r$, we can define the local strain at the surface as

$$
\epsilon_{\mathrm{loc}}(r, \delta)=\sqrt{1+\left(\frac{\mathrm{d} u_{z}}{\mathrm{~d} r}\right)^{2}}-1
$$

and calculate the total energy of the deformed surface by an integral over the energy densities $w_{\phi}(\epsilon)$

$$
U_{\phi}(\delta)=2 \pi \int_{0}^{\infty} d \mathrm{r} r w_{\phi}\left(\epsilon_{\mathrm{loc}}\right) .
$$

The dilution-dependent elastic modulus $\kappa(\phi)$ is then extracted from a quadratic fit of the energy function

$$
U_{\phi}(\delta)=\frac{1}{2} \kappa(\phi) \delta^{2}
$$

to the total deformation energy $U_{\phi}(\delta)$ given by equation (4) and we thus obtain the rescaled effective modulus $\Delta \kappa(\phi)=\kappa(\phi) / \kappa(0)$ ) (Fig. $5 \mathrm{~g}$ ). The single parameter of this model is the collagen modulus $E_{\text {col }}$, in equation (1), which we obtain by a global fit of $\Delta \kappa(\phi)$ over all dilutions $\phi$ to the experimental data. The custom code generated for the theoretical modelling is available at https://doi.org/10.17169/ refubium-28413.

Intracellular optical tweezers-based microrheology. 4T1 and KPC-mT4 were suspended in Matrigel and Net4 or Mut containing Matrigel (Corning) mixtures

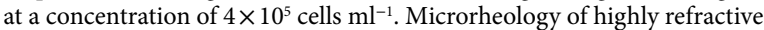
endogenous lipid granules was performed using an optical trap implemented in an inverted Leica DMIRBE microscope as described previously ${ }^{53}$. The laser (Nd:YVO ${ }_{4}, 5 \mathrm{~W}$ Spectra Physics BL106C, $\lambda=1,064 \mathrm{~nm}$, TEM $\infty$ ) was highly focused by a water immersion objective (Leica, HCX PL APO $63 \times / 1.2$ ), collected by a condenser (Leica, P1 1.40 oil S1) and focused onto a quadrant photodiode (S5981, Hamamatsu) detection system. Data were recorded by an acquisition card (NI PCI$6040 \mathrm{E}$ ) at a sampling frequency of $22 \mathrm{kHz}$ and processed by LabVIEW software (LabVIEW 2010, National Instruments). For the small thermal fluctuations of lipid granules the voltage output of the photodiode is linearly related to particle displacement with respect to the laser focus ${ }^{70}$. The positional time series were Fourier transformed and the power spectrum was analysed in Matlab R2016a (MathWorks) as described previously ${ }^{71}$. The movement of an optically trapped particle inside a viscoelastic environment is well described by a modified Langevin equation (6) where, at frequencies above the corner frequency, the positional power spectrum, $P_{x}(f)$, scales with an exponent, $\alpha$ :

$$
\left\langle|\widetilde{x}(f)|^{2}\right\rangle \propto \frac{1}{f^{(1+\alpha)}}
$$

where $f$ denotes frequency. To extract the scaling exponent $\alpha$, the above equation was fitted to the data in the frequency interval $500-9,900 \mathrm{~Hz}$ which is well above the corner frequency. The scaling exponent $\alpha$ carries information about the local viscoelastic landscape $\mathrm{e}^{71,72}$ within living cells, where subdiffusion (characterized by $\alpha<1$ ) is the dominating mode of diffusion. In the subdiffusive regime, a close to 1 indicates a more viscous environment, whereas $\alpha$ closer to 0 describes a more elastic environment.

Statistical analysis. Normality test was performed to determine normal distribution of the acquired data. Based on passing/not passing a normality test, a parametric (two-tailed unpaired $t$ test or ordinary one-way ANOVA) or non-parametric test (two-tailed Mann-Whitney or Kruskal-Wallis) was chosen. In the case of pressure myography experiments, the analysis was performed using a linear regression test. The statistical tests used are indicated in the figure legends. Statistical analysis was performed using Prism version 8.4.3 (GraphPad) unless otherwise stated. Pearson's correlations in the ovarian dataset have been calculated in R version 3.5.1 using the function cor.test. The effect size $r$ of Pearson's correlation is indicated in the figures and legends.

Reporting Summary. Further information on research design is available in the Nature Research Reporting Summary linked to this article.

\section{Data availability}

All data used to generate graphs are available in the respective source data. The generated liquid chromatography-mass spectrometry data are available via the PRIDE database of ProteomeXchange (PXD022145). Data used in this study were taken from previously published datasets including the NKI breast cancer dataset (https://ccb.nki.nl/data/), normal breast data (https://github.com/monkgroupie/ Disease-Specific-Genomic-Analysis), kidney dataset (GSE3538 and GSE3931), CANBUILD dataset (GEO: GSE71340), human melanoma scRNA-seq dataset (GEO: GSE72056), bulk melanoma RNA-seq data via TCGA (Human Skin Cutaneous Melanoma (SKCM)). Source data are provided with this paper.

\section{Code availability}

Custom codes of DSGA analysis of breast and kidney cancer datasets are available at GitHub (https://github.com/monkgroupie/publication_code). The custom code of the mathematical simulation of the impact of node dilution on the laminin network and overall BM stiffness is available via https://doi.org/10.17169/ refubium-28413.

\section{References}

56. Bald, T. et al. Ultraviolet-radiation-induced inflammation promotes angiotropism and metastasis in melanoma. Nature 507, 109-113 (2014).

57. Johnstone, C. N. et al. Functional and molecular characterisation of EO771. LMB tumours, a new C57BL/6-mouse-derived model of spontaneously metastatic mammary cancer. Dis. Model Mech. 8, 237-251 (2015).

58. Aslakson, C. J. \& Miller, F. R. Selective events in the metastatic process defined by analysis of the sequential dissemination of subpopulations of a mouse mammary tumor. Cancer Res. 52, 1399-1405 (1992).

59. Morton, J. P. et al. Dasatinib inhibits the development of metastases in a mouse model of pancreatic ductal adenocarcinoma. Gastroenterology 139, 292-303 (2010)

60. Minn, A. J. et al. Genes that mediate breast cancer metastasis to lung. Nature 436, 518-524 (2005).

61. Calvo, F. et al. Mechanotransduction and YAP-dependent matrix remodelling is required for the generation and maintenance of cancer-associated fibroblasts. Nat. Cell Biol. 15, 637-646 (2013).

62. Kowarz, E., Loscher, D. \& Marschalek, R. Optimized sleeping beauty transposons rapidly generate stable transgenic cell lines. Biotechnol. J. 10, 647-653 (2015)

63. Kulak, N. A., Pichler, G., Paron, I., Nagaraj, N. \& Mann, M. Minimal, encapsulated proteomic-sample processing applied to copy-number estimation in eukaryotic cells. Nat. Methods 11, 319-324 (2014).

64. Schjorring, O. L., Carlsson, R. \& Simonsen, U. Pressure myography to study the function and structure of isolated small arteries. Methods Mol. Biol. 1339, 277-295 (2015)

65. Butt, H. J. \& Jaschke, M. Calculation of thermal noise in atomic force microscopy. Nanotechnology 6, 1-7 (1995).

66. Docheva, D. et al. Researching into the cellular shape, volume and elasticity of mesenchymal stem cells, osteoblasts and osteosarcoma cells by atomic force microscopy. J. Cell. Mol. Med. 12, 537-552 (2008).

67. Prein, C. et al. Structural and mechanical properties of the proliferative zone of the developing murine growth plate cartilage assessed by atomic force microscopy. Matrix Biol. 50, 1-15 (2016).

68. Sneddon, I. N. Boussinesq's problem for a rigid cone. Math. Proc. Camb. Philos. Soc. 44, 492-507 (1948).

69. Sneddon, I. N. The relation between load and penetration in the axisymmetric Boussinesq problem for a punch of arbitrary profile. Int. J. Eng. Sci. 3, 47-57 (1965).

70. Ott, D., Reihani, S. N. \& Oddershede, L. B. Crosstalk elimination in the detection of dual-beam optical tweezers by spatial filtering. Rev. Sci. Instrum. 85, 053108 (2014).

71. Selhuber-Unkel, C., Yde, P., Berg-Sorensen, K. \& Oddershede, L. B. Variety in intracellular diffusion during the cell cycle. Phys. Biol. 6, 025015 (2009).

72. Tolic-Norrelykke, I. M., Munteanu, E. L., Thon, G., Oddershede, L. \& Berg-Sorensen, K. Anomalous diffusion in living yeast cells. Phys. Rev. Lett. 93, 078102 (2004)

\section{Acknowledgements}

We thank N.M. Christensen at the Center for Advanced Bioimaging (CAB), University of Copenhagen for providing microscope access, M. Ghilav and C. Hoffman for valuable experimental help on mouse perfusion and electron microscopy preparation, M.K.G. Høg and Britt Kongstofte for preparation of tissue samples, and L. Amandus and B.L. Kristensen for their support with all aspects of mouse handling. The melanoma 
patient RNA-seq dataset analysed here is in part based upon data generated by the TCGA Research Network: https://www.cancer.gov/tcga. This work was supported by the European Research Council (ERC-2015-CoG-682881-MATRICAN (R.R., M.N., A.E.M.-G. and J.T.E.)); the Danish Cancer Society (R204-A12454 (R.R.), R146-A9250 (A.L.) and R167-A10618 (S.R.N.)); German Cancer Aid (R.R.); a Hallas Møller Stipend from the Novo Nordisk Foundation (J.T.E.); the German Research Foundation (DFG) (NI 1895/1-1 (D.N.), CRC1114/C02 (S.Z. and R.R.N.), FOR2722/B2 (M.K.) and CRC829 (W.B.)); a PhD fellowship from the Lundbeck Foundation (R286-2018-621 (M.R.)); a postdoctoral fellowship from the Lundbeck Foundation (R250-2017-389 (E.M.S.)); a grant from the Novo Nordisk Foundation (Novo Nordisk Foundation Center for Stem Cell Biology, DanStem, NNF17CC0027852 (E.M.S., B.T.P., H.K. and K.J.W.) and NNF19SA0035442 (B.F.)); the Danish Research Council (DFF-4002-00099 (L.W. and L.B.O.)); grants from the UK Medical Research Council (MR/R005567-1 (T.V.A.) and BHF (PG/15/92/31813 (T.V.A.)); a BHF studentship (FS/15/64/32035 (S.M.)); CANTER Research Focus of the Bavarian State Ministry for Science and Education (L.F., S.K., C.P., S.S. and H.C.S.); a CRUK Centre of Excellence Award to Barts Cancer Centre (C16420/ A18066 (E.M.)); a CRUK fellowship (A27947 (O.M.T.P.)); the Ragnar Söderberg Foundation Sweden (N91/15 (C.D.M.)); the Swedish Research Council (2017-03389 (C.D.M.)); Cancerfonden Sweden (CAN 2016/783, 190632 Pj and 190007 (C.D.M.)); the Åke Wiberg Foundation (M17-0235 (C.D.M.)); and the Crafoord Foundation (20171049 (C.D.M.)).

\section{Author contributions}

R.R. hypothesized the mechanism and developed the project idea. R.R. and J.T.E. conceived and designed the study. R.R. defined the BM gene list. R.R., A.L., K.W.Z and S.R.N. performed animal experiments. M.N. performed the genomic association analysis of BM genes with assistance from R.R. S.R.N. performed staining, imaging and analysis of stromal cells within E0771 primary tumours. R.R. performed proliferation experiments. A.L. performed contraction assays. S.Z., R.R.N. and R.R. developed the laminin network stiffness model. S.Z. and R.R.N. performed elastic module simulation of the laminin network. L.F. and H.S.C. performed AFM analysis of the alveolar BM. S.K., C.P. and S.S. performed the AFM analysis of Matrigel. D.N. established the cancer cell invasion assay. D.N. and R.R. produced the recombinant proteins and performed invasion assays. M.R., E.M.S., B.F., B.T.P. and R.R. prepared and performed mass spectrometry experiments. H.K. and K.J.W. performed the scRNA-seq analysis. L.W. and L.B.O. performed optical tweezer experiments. S.M. and T.V.A. performed pressure myography measurements. R.R. and F.S. performed the immunofluorescence staining. E.M. and O.M.T.P. performed the analysis of BM gene association with ovarian patient tissue stiffness. M.K. provided the Net4 knockout mouse model and Net4-specific antibody. A.E.M.-G. performed the decellularization of lung and mesentery. C.D.M. and R.R. performed the imaging of the immunofluorescence staining. W.B. performed transmission electron microscopy analysis. R.R., J.T.E., M.N. and S.Z. wrote the paper. A.E.M.-G. drew the illustrations in Figs. 2a,g, 3b,i, 4a,c and 6b,c. R.R. generated the graphics in Figs. 5a,g and 6a and Extended Data Fig. 10a. R.R. and J.T.E. supervised the project. All authors discussed the results and commented on the manuscript text.

\section{Competing interests}

The authors declare no competing interests.

\section{Additional information}

Extended data are available for this paper at https://doi.org/10.1038/ s41563-020-00894-0.

Supplementary information is available for this paper at https://doi.org/10.1038/ s41563-020-00894-0.

Correspondence and requests for materials should be addressed to R.R. or J.T.E.

Peer review information Nature Materials thanks P. Mehlen, P. Yurchenco and the other, anonymous, reviewer(s) for their contribution to the peer review of this work.

Reprints and permissions information is available at www.nature.com/reprints. 
a

Disease component vs. Normal component vs. nonDSGA of basement membrane genes in breast cancer patients

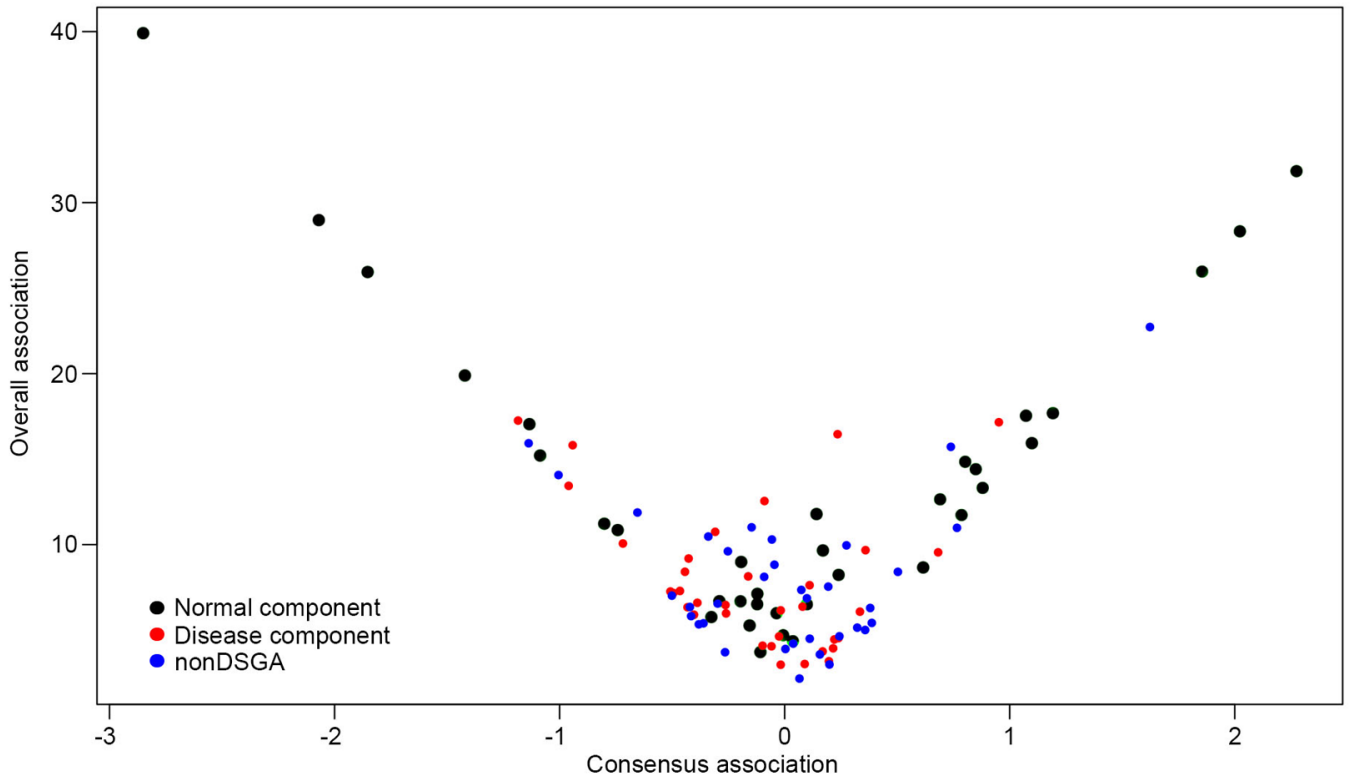

b

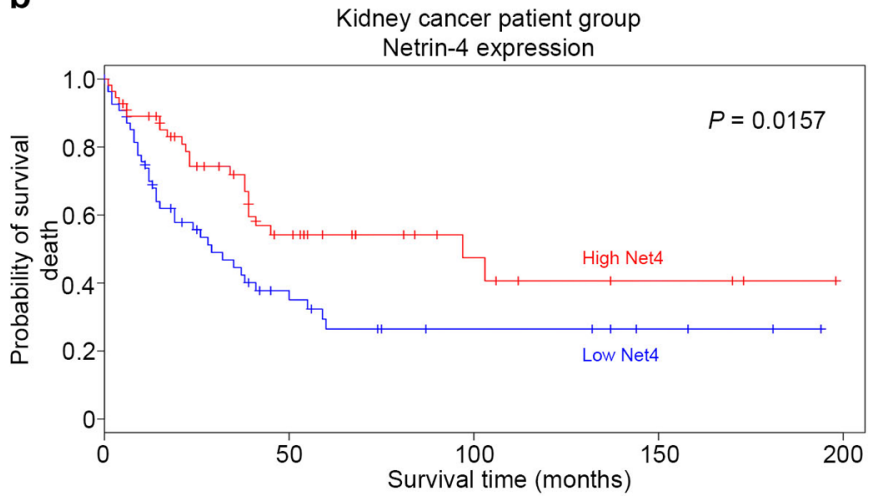

C Kidney cancer patient group

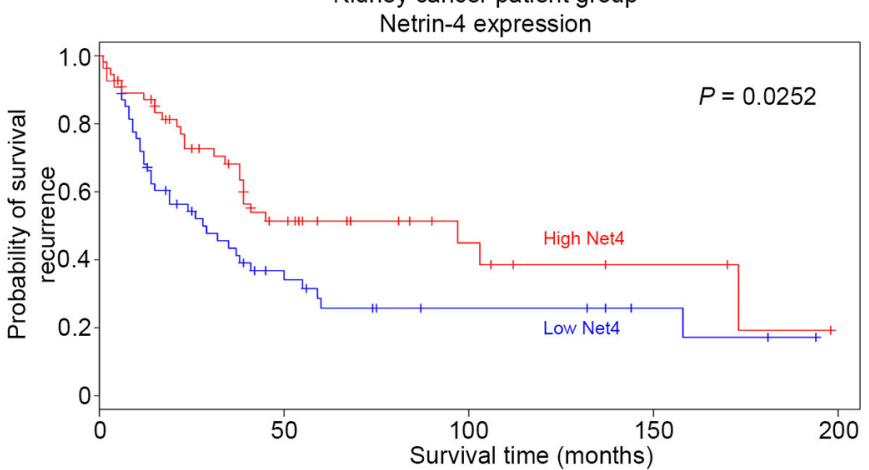

Extended Data Fig. 1 | Distribution of Normal versus Disease components in breast cancer patients and association of Net4 with kidney cancer patient survival. a, Plots of consensus vs. overall associations in the Disease components, the Normal components, and the nonDSGA data. Most significant association with survival is captured in the Normal component of data. $\mathbf{b}$, Kaplan Meier survival (death) analysis using Cox proportional hazard models for association with survival of NTN4 expression levels in the Normal component of renal cell carcinoma. Survival analysis comparison of (tumor group 1) low levels of NTN4 (33 percentile) vs. (tumor group 2) high levels of NTN4 (67 percentile) in the Normal component of data. High levels of NTN4 show significant association with good survival with $P$ value 0.0157. c, Kaplan Meier survival (recurrence) analysis for association with survival of NTN4 levels in the Normal component of renal cell carcinoma. Survival analysis comparison of (tumor group 1) low levels of NTN4 (33 percentile) vs. (tumor group 2) high levels of NTN4 (67 percentile) in Normal component of data. High levels of NTN4 showed significant association with good survival with P value 0.0252. 
a
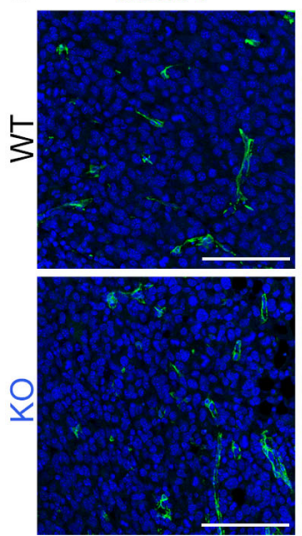

b
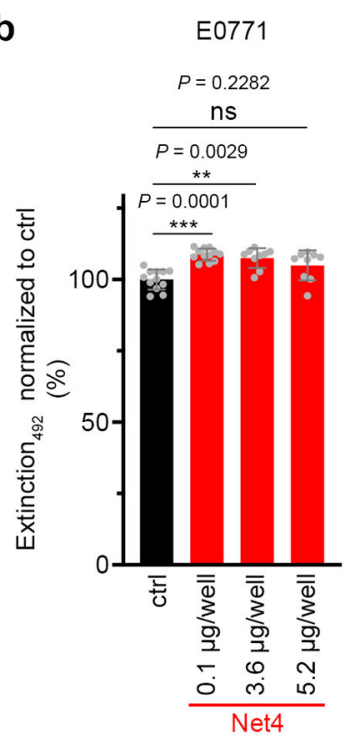

C

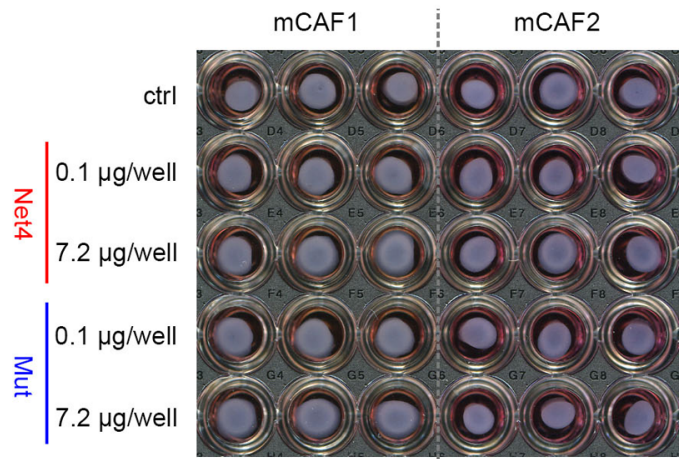

CD8a
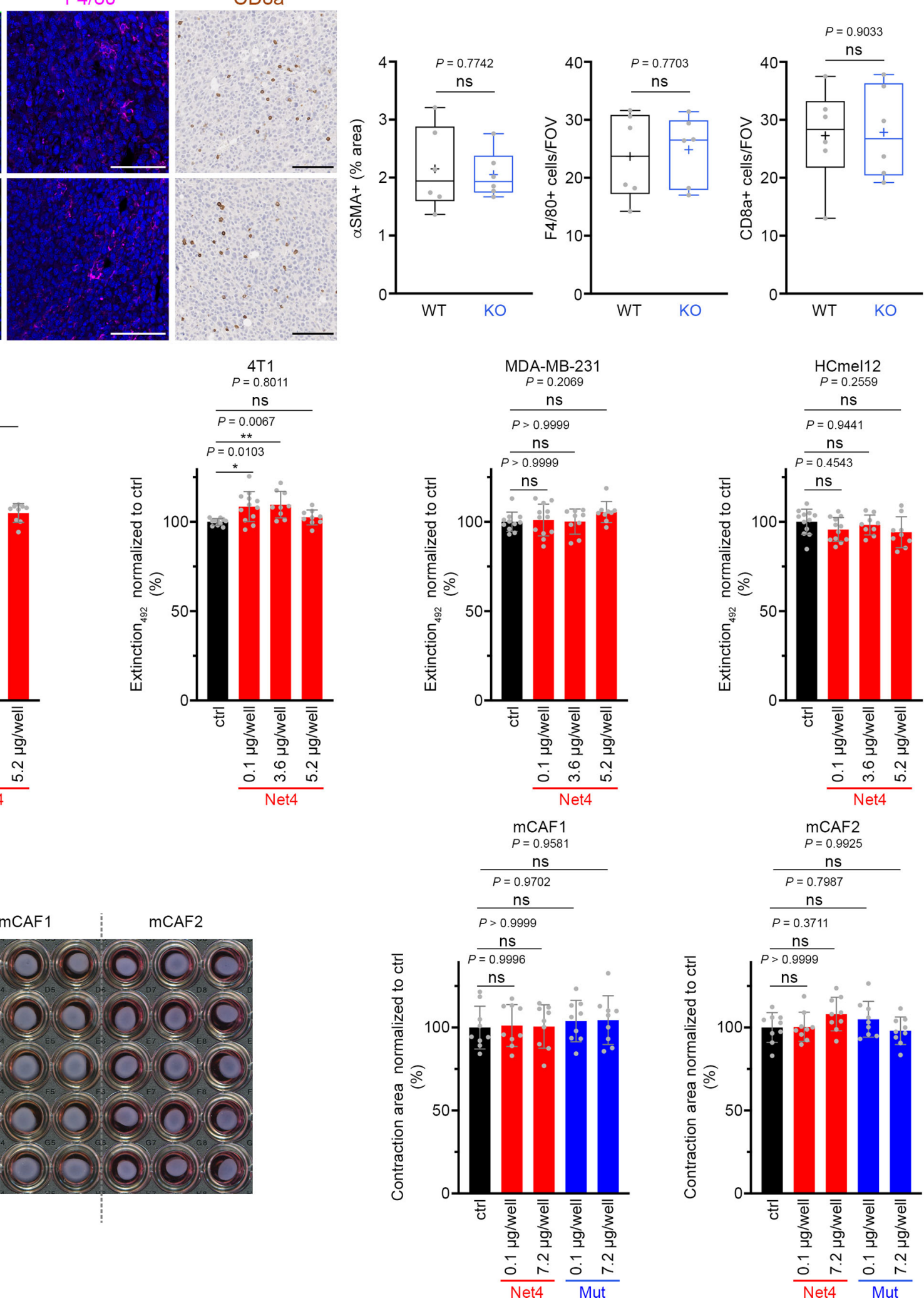
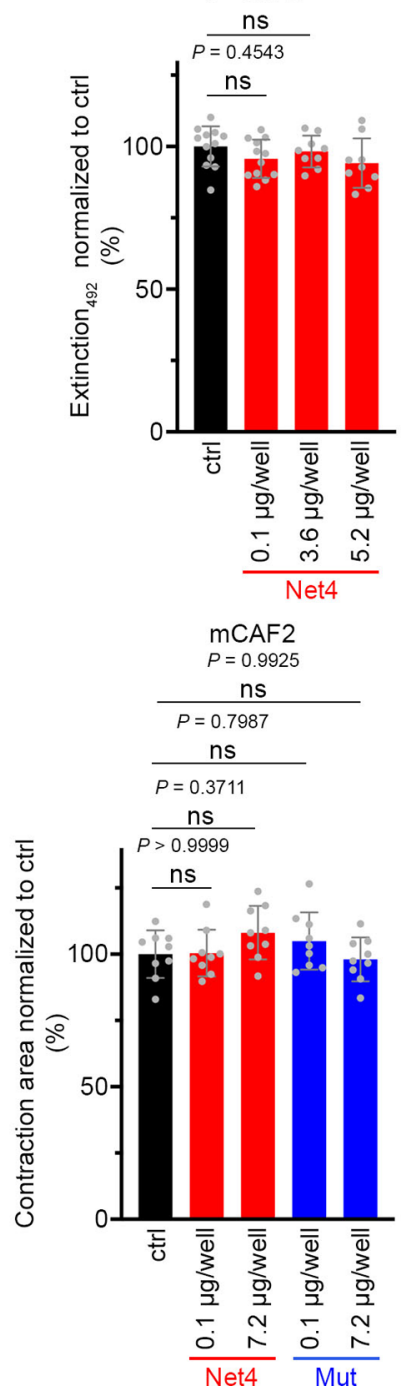

Extended Data Fig. 2 | See next page for caption. 
Extended Data Fig. 2 | Validation of E0771 primary tumor from Net4 knockout and wildtype mice. a, Immunofluorescence and immunohistochemistry staining of primary E0771 tumors grown in Net4 wildtype (WT) and Net4 knockout (KO) animals. Different stromal cell types were detected using $\alpha \mathrm{SMA}$ (fibroblasts), F4/80 (macrophages), and CD8a (T-cells). Scale bar, $100 \mu \mathrm{m}$. Box and whiskers blot of cell type amount within primary tumor tissues (two-tailed Unpaired t test, Min to Max, median is shown as line, mean is displayed as cross, data points appear as grey dots; $n=6 ; t=0.2948$ ( $\alpha \mathrm{SMA}$ ), $t=0.3001(F 4 / 80), t=0.1246(C D 8 a), d f=10 ; n s$, not significant). b, Proliferation of cancer cell lines (E0771, 4T1, MDA-MB-231, and HCmel12) treated without (ctrl) and with different concentrations of $\operatorname{Net} 4(0.1,3.6,5.2 \mu \mathrm{g} /$ well) for $96 \mathrm{~h}$. Absorbance was normalized to ctrl. Scatter dot blot together with bars of proliferation assay (E0771: Kruskal-Wallis test, mean is the top of the bar; STDEV; $n=12$ (ctrl and $0.1 \mu g / w e l l), n=9$ (3.6 and $5.2 \mu \mathrm{g} / \mathrm{well}) ; \mathrm{ns}$, not significant; $\left.{ }^{\star \star} P<0.01,{ }^{\star \star \star} P<0.001\right),(4 T 1$, MDA-MB-231, and HCmel12: Ordinary one-way ANOVA test, mean is the top of the bar; $n=11$ (4T1 and MDA-MB-231: ctrl), $n=12$ (HCmel12: ctrl), $n=9$ (3.6 and 5.2 $\mu \mathrm{g} /$ well); $F=5.938, \mathrm{DF}=37(4 \mathrm{~T} 1) ; \mathrm{F}=1.254, \mathrm{DF}=37(\mathrm{MDA}-\mathrm{MB}-231) ; \mathrm{F}=1.430, \mathrm{DF}=38$ (HCmel12); ns, not significant; $\left.{ }^{\star} P<0.05,{ }^{\star \star} P<0.01\right)$. c, Contraction assay of cancer-associated fibroblast cell lines (mCAF1 and mCAF2) within collagen c1 and $7.2 \mu \mathrm{g} /$ well) of recombinant Net4 (Net4) and its respective laminin binding mutant Net4 E195A,R199A (Mut) for $72 \mathrm{~h}$. Representative image of the contraction assay showing the contracted area at the well rim. Scatter dot blot together with bars of contraction assay, in which all values are normalized to the control (Ordinary one-way ANOVA test, mean is the top of the bar; STDEV; $n=9$; ns, not significant). 


\section{NATURE MATERIALS}
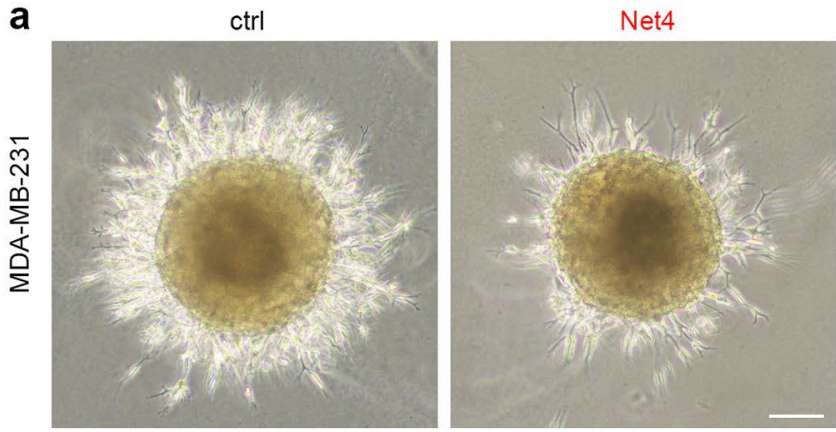

ctrl

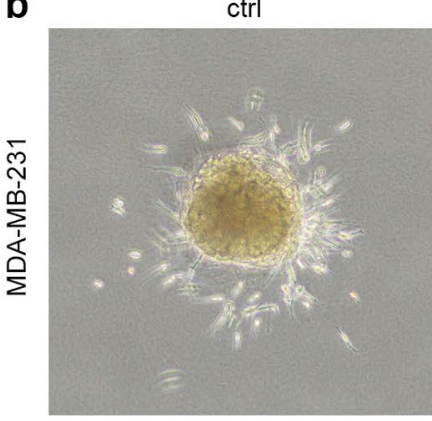

Net4

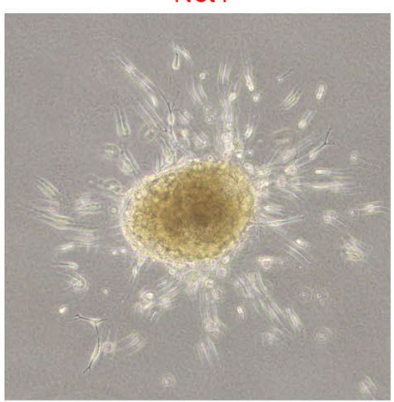

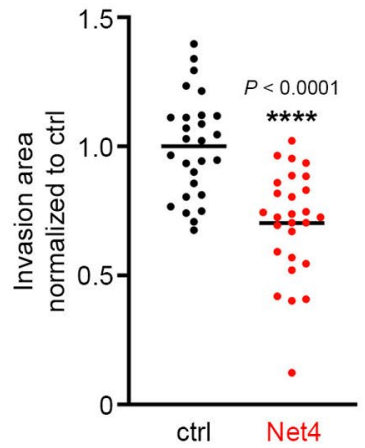

Mut

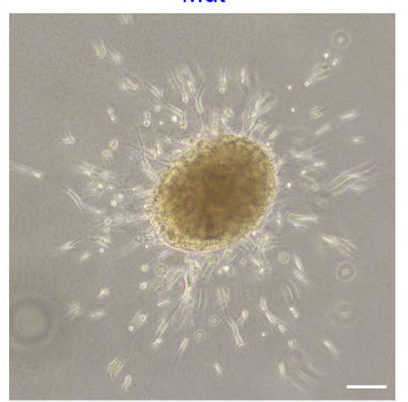

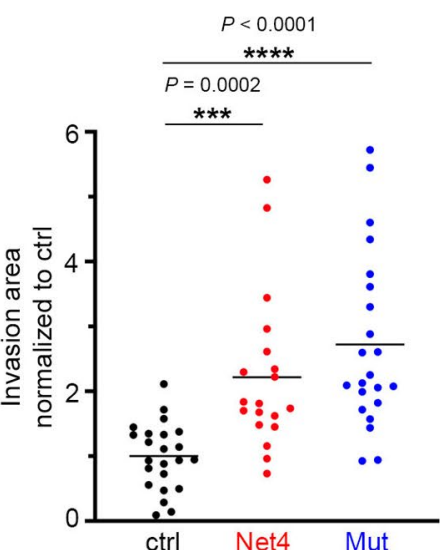

Extended Data Fig. 3 | Breast cancer cell sphere invasion into Matrigel supplemented with Net4. a, Representative images of sphere invasion assay using MDA-MB-231 cells in Matrigel (ctrl) and Matrigel containing 25\% Net4 molecules (Net4) relative to laminin 111 within the Matrigel matrix. Scale bar, $100 \mu \mathrm{m}$. Statistical analysis of sphere invasion (two-tailed Unpaired t test, Scatter dot blot, mean is displayed as black line; $n=27$ of each sphere normalized to the mean of all ctrl spheres; $t=5.386, \mathrm{df}=52$; $\left.{ }^{\star \star \star \star} P<0.0001\right)$. b. Representative images of sphere invasion assay using MDA-MB-231 cells in $1.5 \mathrm{mg} / \mathrm{ml}$ collagen I (ctrl) and in $1.5 \mathrm{mg} / \mathrm{ml}$ collagen I containing $5.2 \mu \mathrm{g} / \mathrm{sphere} \mathrm{Net4} \mathrm{(Net4)} \mathrm{or} 5.2 \mu \mathrm{g} / \mathrm{sphere} \mathrm{Net4} \mathrm{laminin} \mathrm{binding} \mathrm{mutant} \mathrm{(Mut)}$ (Net4 molecule number equal to number in $50 \%$ Net4 Matrigel sphere). Scale bar, $100 \mu \mathrm{m}$. Statistical analysis of sphere invasion (Kruskal-Wallis test, Scatter dot blot, median is shown as line; $n=23$ (ctrl and Mut) and $n=20$ (Net4); ${ }^{\star \star \star} P<0.001$, ${ }^{\star \star \star \star} P<0.0001$ ). 
a

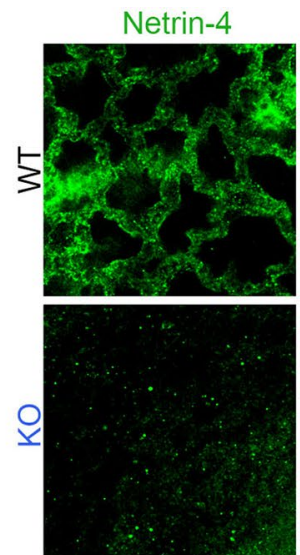

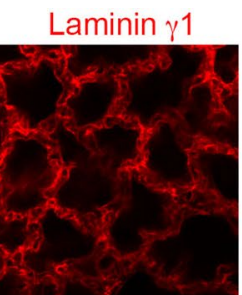

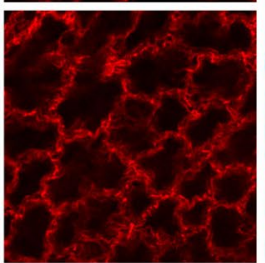

Collagen IV

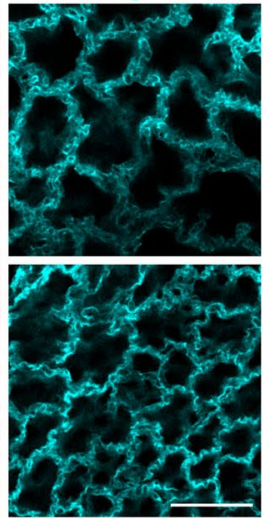

b

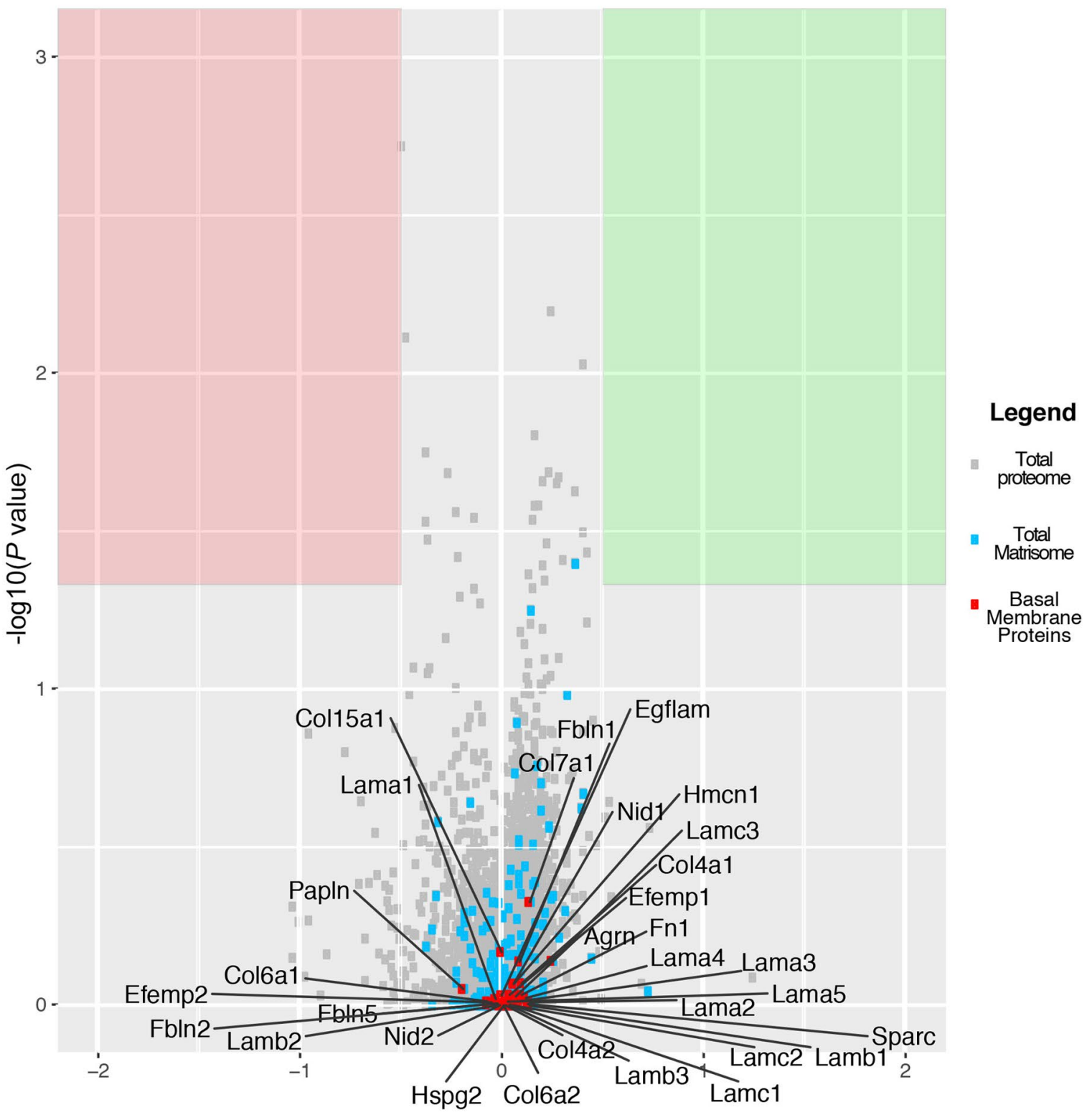

Log2 Ratio KO vs. WT

$\mathrm{N}=5,359$ proteins

Extended Data Fig. 4 | See next page for caption. 
Extended Data Fig. 4 | Validation of Net4 knockout mouse. a, Immunofluorescence staining of decellularized lungs from Net4 WT and Net4 KO animals for Net4 (green), laminin $\gamma 1$ (red), and collagen IV (cyan). Representative images of lung tissues from 3 mice of each genotype. Scale bar, $50 \mu$ m. b, Volcano plot of all identified proteins within five lung tissues from each Net4 WT and KO mice from the whole proteome LC-MS analysis. The plot visualizes the comparison of the protein abundance in lung tissues from WT vs. KO mice ( $x$-axis: $\log _{2}$ ratio KO vs. WT; $y$-axis: $-\log _{10} P$ value). The red box highlights proteins with a $\log _{2}$ ratio KO vs. WT below -0.5 and the green box above 0.5 together with a $P$ value above 0.05 (- $\log _{10} p$-value 1.3$)$. Both boxes indicate proteins with significant differential expression and relevant effect sizes between Net4 WT and KO lung tissue. Proteins, which belong to the total proteome, are shown as grey squares. Matrisome proteins are marked as blue squares and identified BM proteins (see Supplementary Table 1) as red squares. The volcano plot emphasizes that there are no BM proteins significantly changed between Net4 WT and KO mouse lung tissues. 

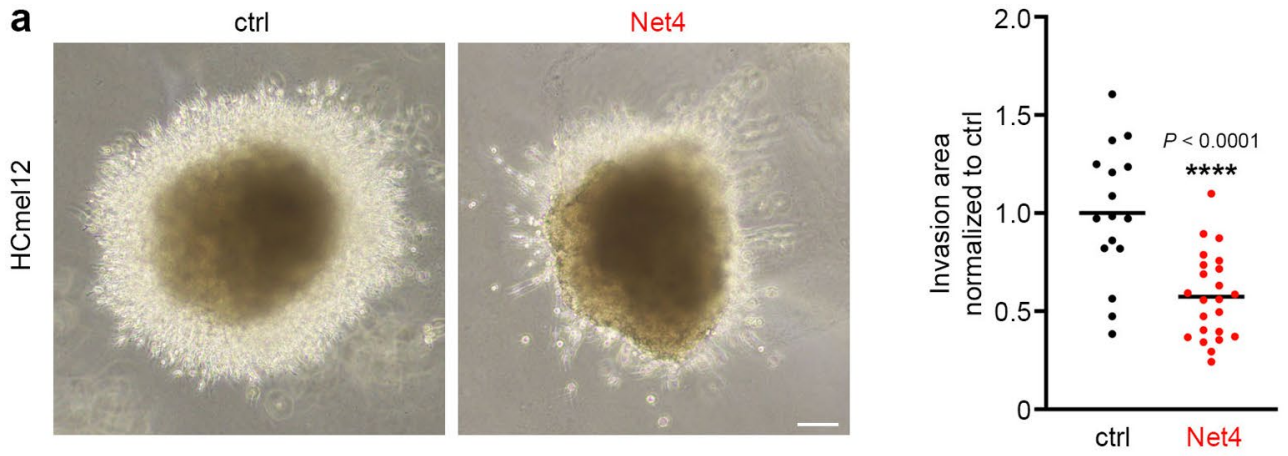

b

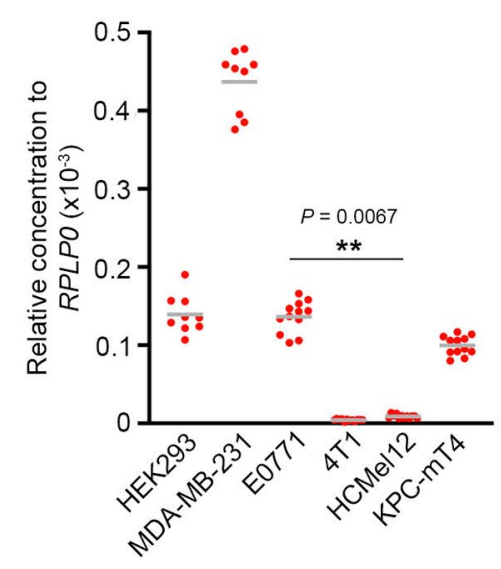

C

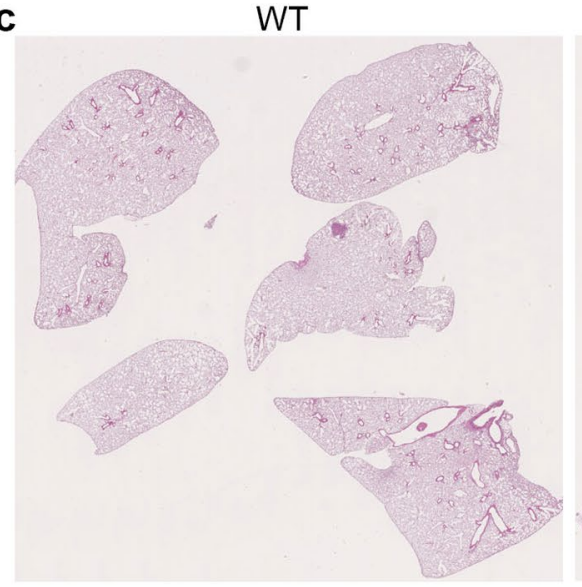

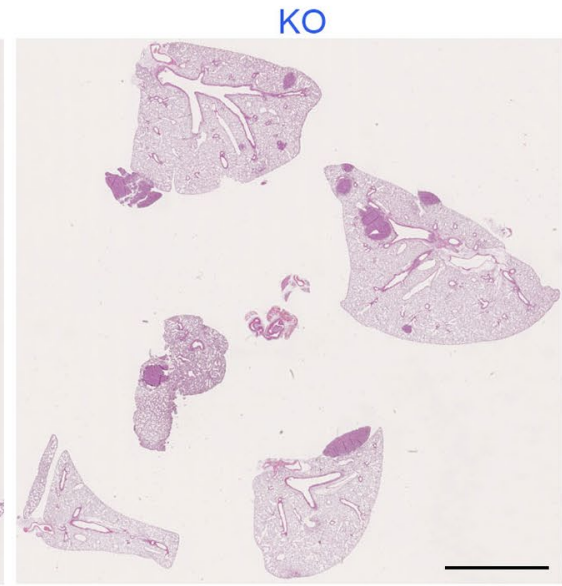

d

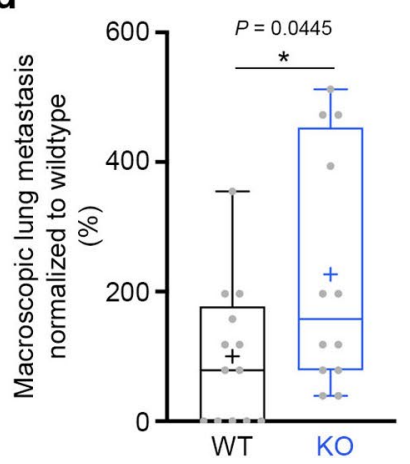

e

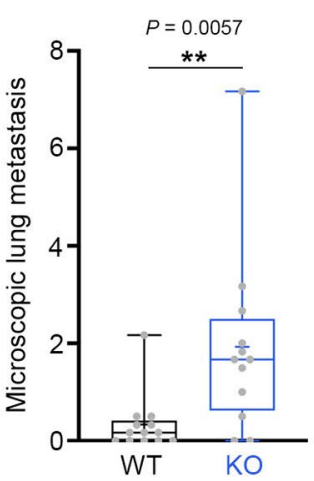

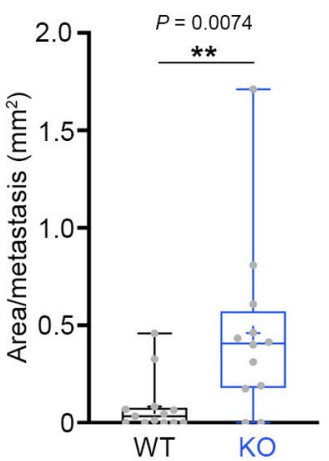

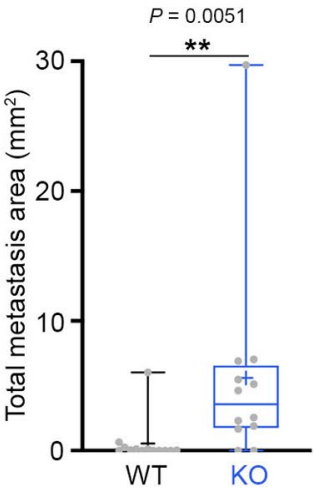

Extended Data Fig. 5 | Net4 deficient pulmonary BM favors metastases formation independent of endogenous Ntn4 expression. a, Representative images of sphere invasion assay using $\mathrm{HC}$ el12 cells in Matrigel (ctrl) and Matrigel containing $25 \%$ Net4 molecules (Net4) relative to laminin 111 within the Matrigel matrix. Scale bar, $100 \mu \mathrm{m}$. Statistical analysis of sphere invasion (two-tailed Unpaired t test, Scatter dot blot, mean is displayed as black line; $\left.n=16(\mathrm{ctrl}), n=23(\mathrm{Net} 4) ; \mathrm{t}=4.728, \mathrm{df}=37 ;{ }^{\star \star \star \star} P<0.0001\right)$. b, Quantitative real time PCR determining the relative concentration of mouse and human Net4 (Ntn4 and NTN4, respectively) expression to the housekeeping gene RPLPO in different cell lines used in this study (HEK293, MDA-MB-231, E0771, 4T1, HCMel12, and KPC-mT4). Statistical analysis of expression levels (Kruskal-Wallis test, Scatter dot blot, median is shown as line; $n=12 ;{ }^{\star \star} P<0.01$ ). c, Representative H\&E images (from mice with the mean metastasis number and area) from Net4 WT and Net4 KO mice 28 days after intravenous injection of E0771 Ntn4 CRISPR/Cas9 knockout cells. Scale bar, $3 \mathrm{~mm}$. d. Statistical analysis of macroscopic lung metastases (two-tailed Unpaired t test, Box and whiskers blot, Min to Max, median is shown as line, mean is displayed as cross, all data points appear as grey dots; $n=13(\mathrm{WT}), n=12(\mathrm{KO})$; $\left.\mathrm{t}=2.126, \mathrm{df}=23 ;{ }^{\star} P<0.05\right) \mathbf{e}$, Statistical analysis of microscopic lung metastasis, area/metastasis, and total metastasis area of intravenous injection of Ntn4 deficient E0771 cells into Net4 WT and Net4 KO mice after 28 days (two-tailed Mann-Whitney test, Box and whiskers blot, Min to Max, median is shown as line, mean is displayed as cross, all data points appear as grey dots; $\left.n=13(\mathrm{WT}), n=12(\mathrm{KO}) ;{ }^{\star \star} p<0.01\right)$. 
a

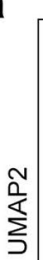

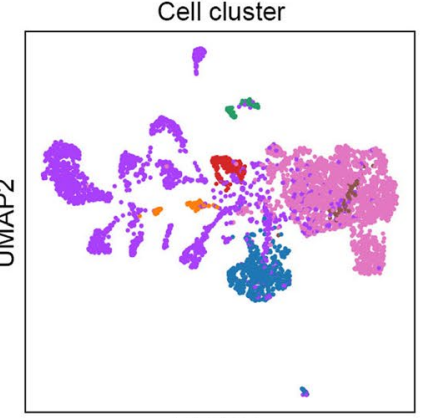

UMAP1 b

B-cells

- CAF

- Endothelial cells

- Macrophages

- Melanoma cells

- NK-cells

- T-cells

Extended Data Fig. 6 | scRNA-seq analysis in melanoma patients. a, UMAP plot highlights cell clusters (B-cells, cancer-associated fibroblasts (CAFs), endothelial cells, macrophages, melanoma cells, NK-cells, and T-cells) of 4,645 cells from 19 melanoma tumor patients. b, UMAP plot indicating cell type-specific NTN4 expression. c, UMAP plot of cell type-specific expression over the 49 BM genes. 

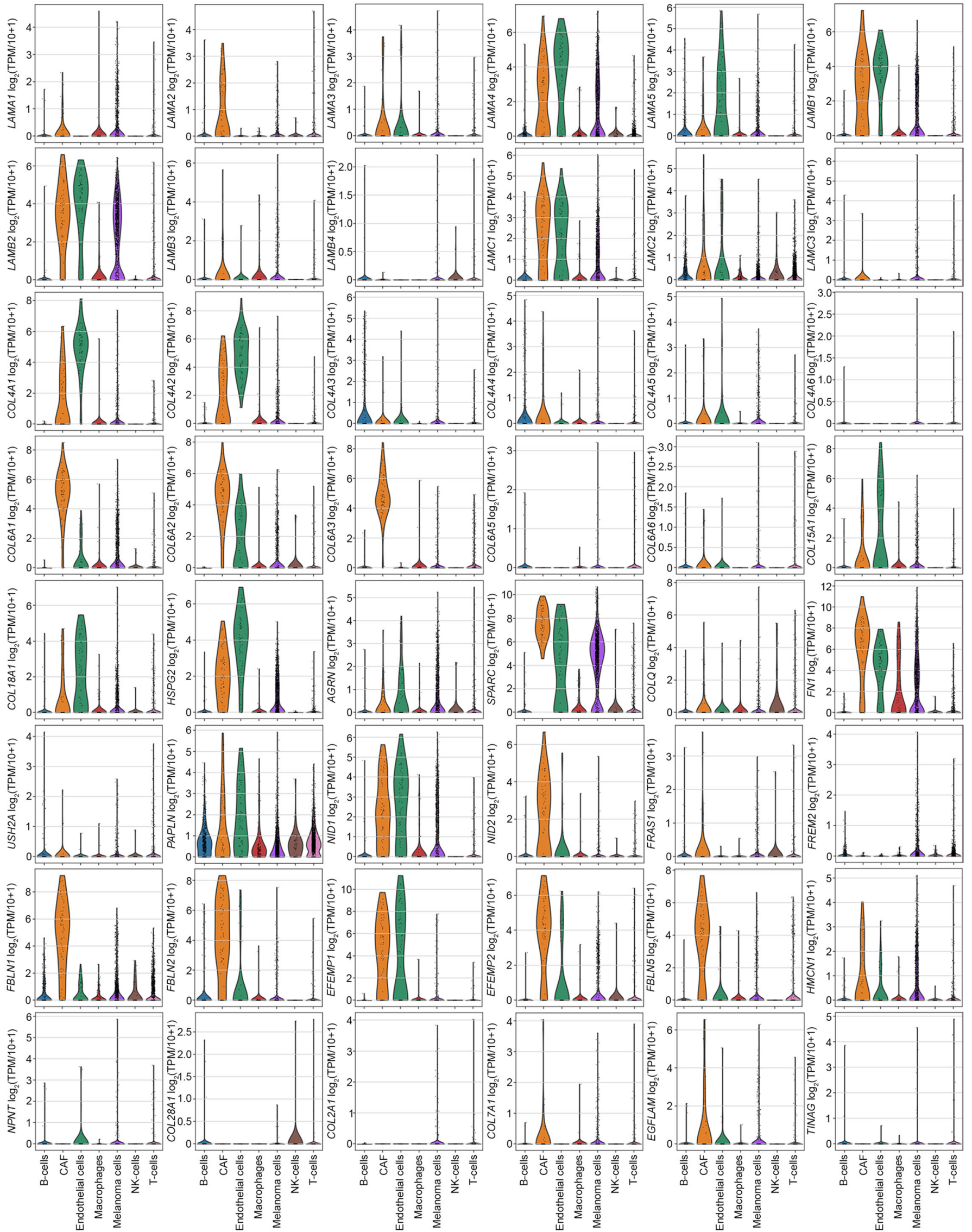

Extended Data Fig. 7 | scRNA-seq analysis of BM protein encoding genes in melanoma patients. Violin plots of 49 BM genes using scRNA-seq from 19 melanoma tumor patients. 
a
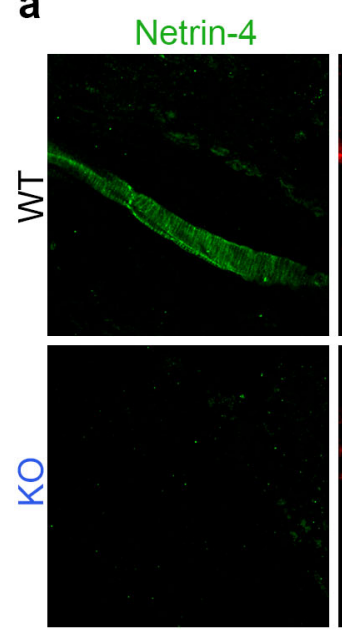

Laminin $\gamma 1$
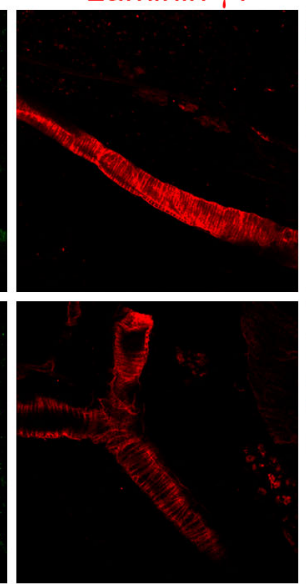

Collagen IV
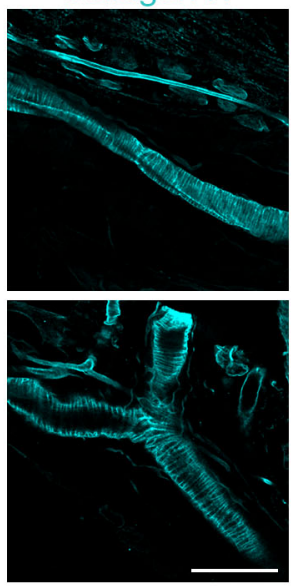

b

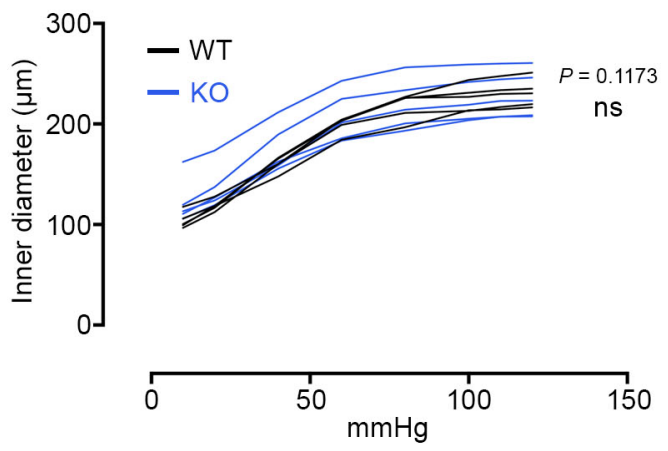

C

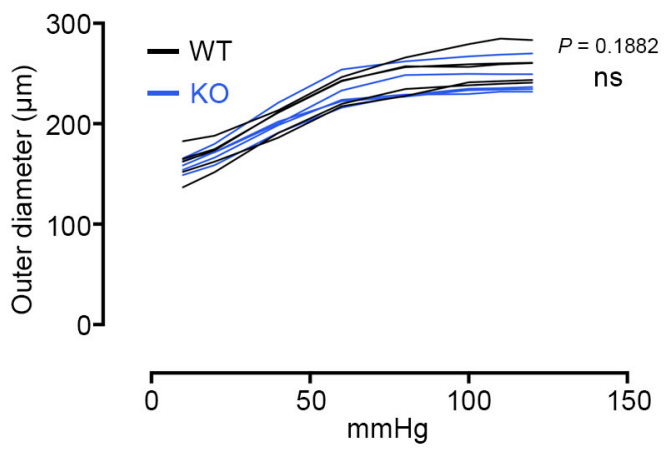

Extended Data Fig. 8 | Impact of Net4 deficiency on mesenteric vessels. a, Immunofluorescence staining of decellularized mesentery showing mesenteric vessels from Net4 WT and Net4 KO animals stained for Net4 (green), laminin $\gamma 1$ (red), and collagen IV (cyan). Representative images of mesenteric vessels from 2 mice of each genotype. Scale bar, $100 \mu \mathrm{m}$. b. Inner diameter ( $\mu \mathrm{m}$ ) of isolated mesenteric arteries from 5 months old Net4 WT and $\mathrm{KO}$ mice as measured by pressure myography (Linear regression test, mean \pm STDEV; $n=5$ individual mice per group; ns, not significant). c, Outer diameter $(\mu \mathrm{m})$ of isolated mesenteric arteries from 5 months old Net4 WT and KO mice as measured by pressure myography (Linear regression test, mean \pm STDEV; $n=5$ individual mice per group; ns, not significant). 

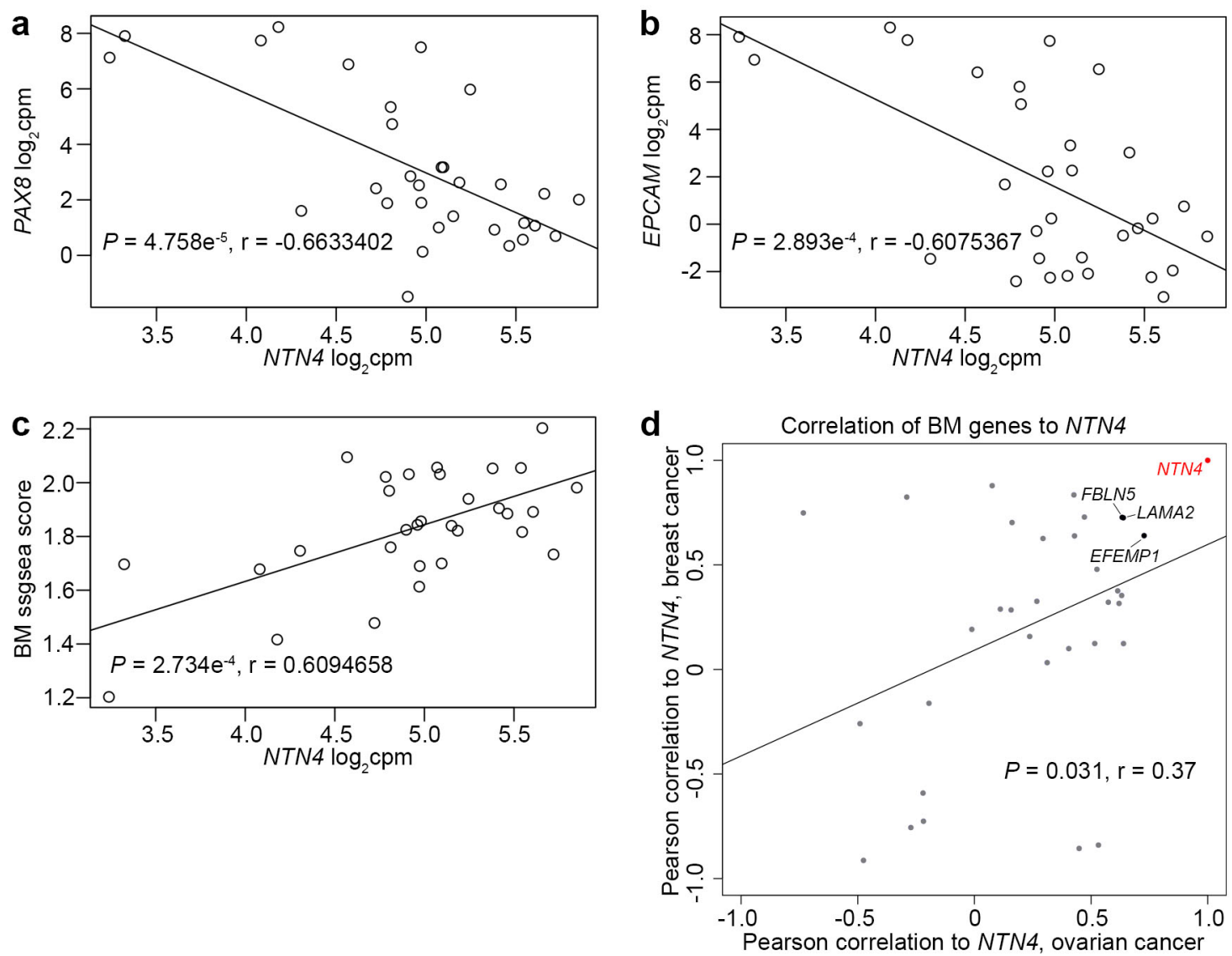

Extended Data Fig. 9 | Correlation of NTN4 expression with malignant and BM markers. a, Graph depicts the correlation between the Net4 (NTN4) gene expression level and the expression level of the malignant marker PAX8 in 31 ovarian cancer patients (Pearson's $p$-value $=4.758 \mathrm{e}^{-5}, \mathrm{r}=-0.6633402$ ). b. Graph depicts the correlation between the Net4 (NTN4) gene expression level and the expression level of the malignant marker EPCAM in 31 ovarian cancer patients (Pearson's $p$-value $=2.893 \mathrm{e}^{-4}, r=-0.6075367$ ). c, Graph depicts the correlation between the Net4 (NTN4) gene expression level and the expression level of BM encoding genes (BM ssgsea score, calculated using R package GSVA) in 31 ovarian cancer patients (Pearson's $p$-value $=2.734 \mathrm{e}^{-4}$, $r=0.6094658$ ). These graphs highlight a negative correlation of NTN4 expression with malignant cell markers and a positive correlation with BM encoding genes in ovarian cancer patients. d, Graph highlights the correlation of BM encoding genes with NTN4 expression levels within ovarian cancer patients ( $x$-axis) to the Normal component of breast cancer patients ( $y$-axis) (Pearson's $p$-value $=0.031, r=0.37$ ). BM genes significantly correlating with NTN4 expression in both cancer types are indicated with black dots (Laminin $\alpha 2$ (LAMA2), fibulin-5 (FBLN5), and fibulin-3 (EFEMP1)). 

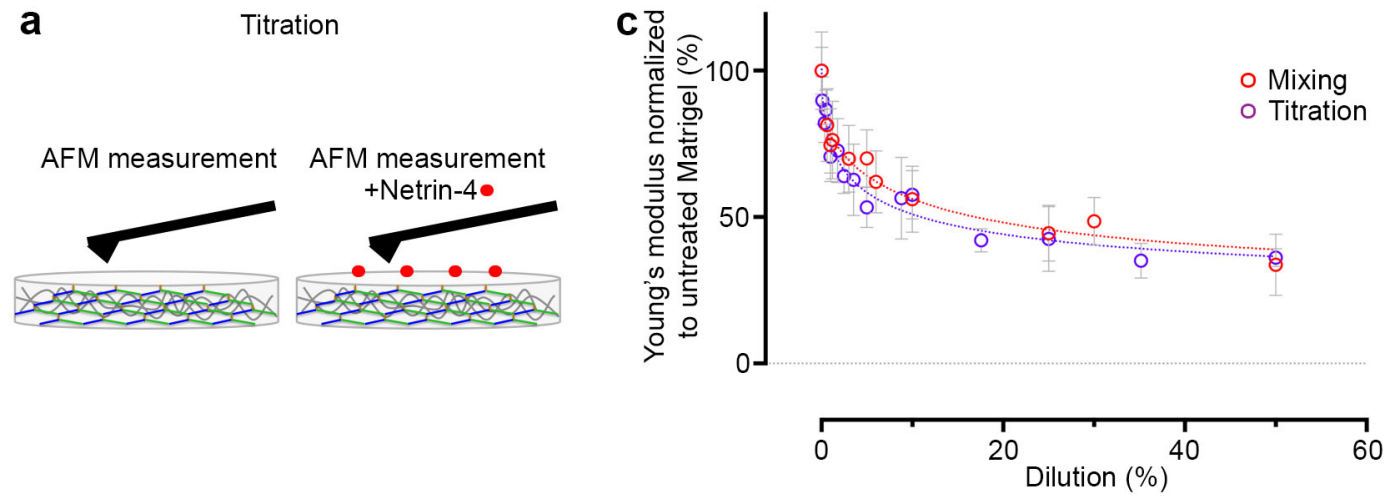

b

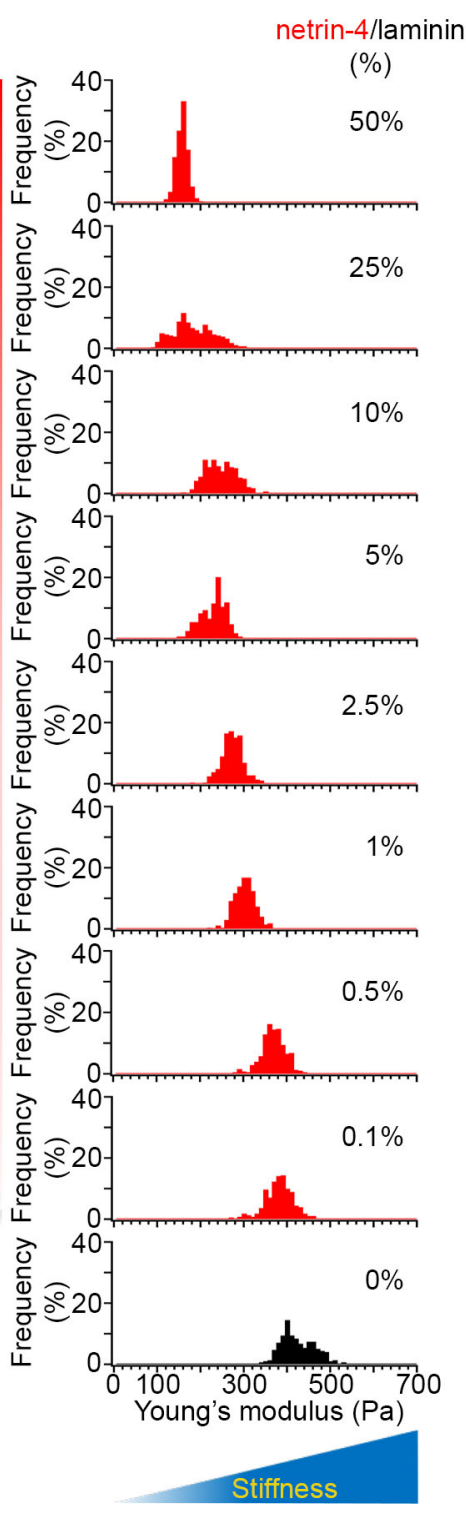

d

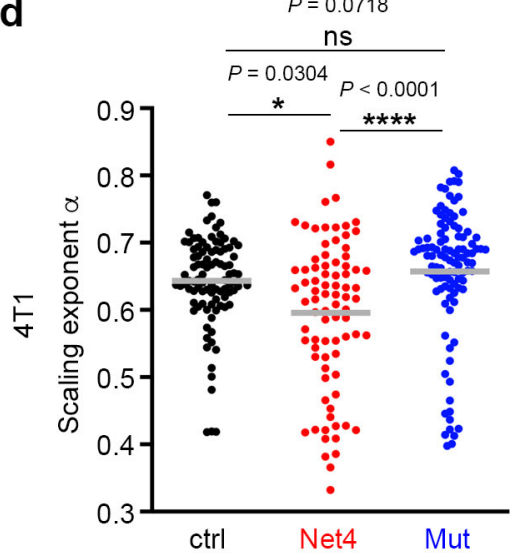

e

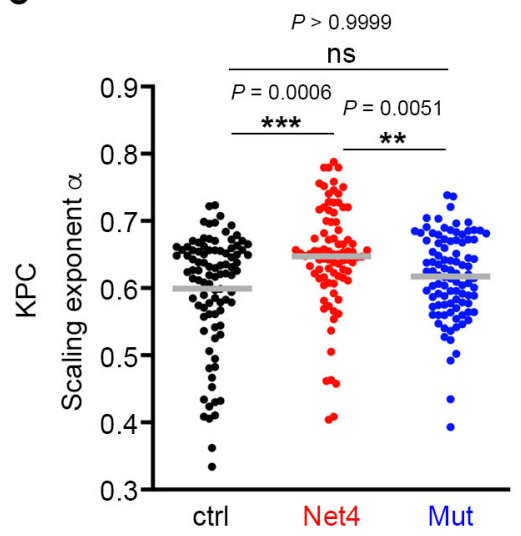

f

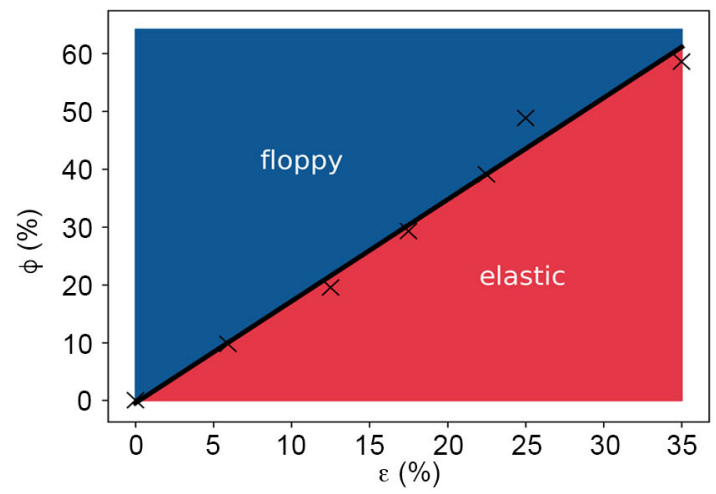

g

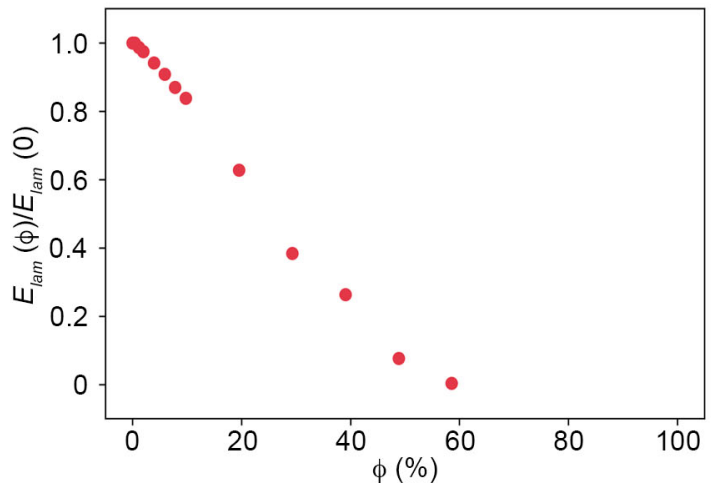

Extended Data Fig. 10 | See next page for caption. 
Extended Data Fig. 10 | Laminin network dilutions impact stiffness. a, Scheme of the AFM experiment to determine the Young's modulus of Matrigel titrated with increasing Net4 amounts (red dots). b. Histograms reveal the Young's modulus of measurements of pure Matrigel (black) and of increasing amounts (\%) of Net4 molecules to laminin 111 molecules inside the Matrigel matrix (red). The amount of Net4 molecule addition is based on the ratio between Net4 and laminin 111 molecules in \%. c, Graph displays data points for the stiffness change (\%) of Matrigel plotted against laminin node dilution (\%) obtained through the AFM measurement of different amounts of Net4 inside the Matrigel (Mixing) and the titration of Matrigel with increasing Net4 amounts (Titration). d, Scatter dot blot of the scaling exponent $\alpha$ of endogenous tracer movement measured by optical tweezers quantifying cytoplasmic visco-elastic properties of $4 \mathrm{~T} 1$ cells inside Matrigel (ctrl), Matrigel supplemented with $50 \%$ Net4 (Net4) or with $50 \%$ Mut (Mut) ${ }^{\star \star \star \star \star(K r u s k a l-W a l l i s ~}$ test, mean is displayed as grey line; $n=96$ (ctrl), $n=82$ (Net4), $n=104$ (Mut); ns, not significant; ${ }^{\star} P<0.05$, ${ }^{\star \star \star \star} P<0.0001$ ). e, Scatter dot blot of the scaling exponent $\alpha$ of endogenous tracer movement measured by optical tweezers quantifying cytoplasmic visco-elastic properties of measurements of KPC cells inside Matrigel (ctrl), Matrigel supplemented with 50\% Net4 (Net4) or with 50\% Mut (Mut) (Kruskal-Wallis test, mean is displayed as grey line; $n=94$ (ctrl), $n=86$ (Net4), $n=97$ (Mut); ns, not significant; ${ }^{* \star} P<0.01$, ${ }^{\star \star \star} P<0.001$ ). f, Phase diagram showing the transition of the diluted honeycomb elastic networks from the floppy to the elastic state under increasing extensional strain for different dilution fractions. $\mathbf{g}$, Rescaled elastic modulus $E_{\text {lam }}(\phi) / E_{\text {lam }}(0)$ extracted from simulations of the $2 \mathrm{D}$ diluted honeycomb network. 


\section{natureresearch}

\section{Reporting Summary}

Nature Research wishes to improve the reproducibility of the work that we publish. This form provides structure for consistency and transparency in reporting. For further information on Nature Research policies, see Authors \& Referees and the Editorial Policy Checklist.

\section{Statistics}

For all statistical analyses, confirm that the following items are present in the figure legend, table legend, main text, or Methods section.

n/a Confirmed

$\bigotimes$ The exact sample size $(n)$ for each experimental group/condition, given as a discrete number and unit of measurement

Х $\square$ A statement on whether measurements were taken from distinct samples or whether the same sample was measured repeatedly

$\varnothing$ The statistical test(s) used AND whether they are one- or two-sided

Only common tests should be described solely by name; describe more complex techniques in the Methods section.

Х $\square$ A description of all covariates tested

Х $\square$ A description of any assumptions or corrections, such as tests of normality and adjustment for multiple comparisons

$\square$ A full description of the statistical parameters including central tendency (e.g. means) or other basic estimates (e.g. regression coefficient)

AND variation (e.g. standard deviation) or associated estimates of uncertainty (e.g. confidence intervals)

For null hypothesis testing, the test statistic (e.g. $F, t, r$ ) with confidence intervals, effect sizes, degrees of freedom and $P$ value noted

Give $P$ values as exact values whenever suitable.

Х $\square$ For Bayesian analysis, information on the choice of priors and Markov chain Monte Carlo settings

Х $\square$ For hierarchical and complex designs, identification of the appropriate level for tests and full reporting of outcomes

$\square \bigotimes$ Estimates of effect sizes (e.g. Cohen's $d$, Pearson's $r$ ), indicating how they were calculated

Our web collection on statistics for biologists contains articles on many of the points above.

\section{Software and code}

Policy information about availability of computer code

Data collection -H\&E and immunohistochemistry slides were imaged using a Hamamatsu Photonics slide scanner with NanoZoomer Digital Pathology (NDP) Scan software; NDP.scan version 2.5.90

-Sphere invasion was imaged using brightfield microscope (Olympus CKX53 with Olympus DP22 camera) with Olympus cellSens Entry software version v.1.16

-Pressure myography data were acquired using 110P system; Danish Myo Technology (DMT), software DMT Myoview (V3)

-Immunofluorescence images were imaged using an SP8 confocal microscope (Leica), software Leica Application Suite X (LAS X) version 3.5.5.19976

-Decellularized tissue stainings were imaged using SP5-X MP 2-photon confocal microscope (Leica), software Leica Application Suite X

(LAS X) version 2.7.4.10100

-LC-MS data acquisition was done using Tune 1.1 and Xcalibur v.4.3

-qRT-PCR data were conducted using a LightCycler 480 II (Roche); Light Cycler 480 SW version 1.5.1

-AFM was conducted using NanoWizard 1 (JPK Instruments) with the JPK Control Software (Version 5.0.130). Histograms in Figure 5b and Extended Data Figure 10b were generated using Igor Pro (WaveMetrics, Version 6.3.7.2).

-Electron microscopy images were conducted using EM 109 Zeiss; Camera: TRS USB (2048×2048, v.596.0/466.0) Fa.Zeiss900N; Software: ImageSP ver.1.2.6.11 (x64)

-Extinction values in the proliferation assay were acquired using a SpectraMax Paradigm plate reader from Molecular Devices, SoftMax Pro6.5.1 software

-Optical tweezer data were acquired using inverted Leica DMIRBE microscope (LabVIEW 2010 [National Instruments]) 
-qRT-PCR was analyzed using the LightCycler 480 SW 1.5.1 software

-AFM data were analyzed with the JPK software (Version 6.0.69)

-Immunohistochemistry was analyzed using ImageJ (Fiji Version 2.1.0/1.53c)

-Mathematical simulations were performed using Python 3.7.3 programming language and the SciPy libraries 1.3.0 and Fortran95

Language (GCC 8.3)

-Optical tweezer data were acquired using Matlab Version R2016a (MathWorks)

-Normalization of raw values were done using Microsoft Excel for Mac version 16.42

-All statistical data were performed using GraphPad Prism 8.4.3

For manuscripts utilizing custom algorithms or software that are central to the research but not yet described in published literature, software must be made available to editors/reviewers. We strongly encourage code deposition in a community repository (e.g. GitHub). See the Nature Research guidelines for submitting code \& software for further information.

\section{Data}

Policy information about availability of data

All manuscripts must include a data availability statement. This statement should provide the following information, where applicable:

- Accession codes, unique identifiers, or web links for publicly available datasets

- A list of figures that have associated raw data

- A description of any restrictions on data availability

Raw data used in this study were taken from previously published datasets

-CANBUILD dataset: GEO: GSE71340

-sCRNAseq dataset: GEO: GSE72056

-Melanoma dataset: bulk RNA-seq data (PMID 26091043), TCGA: Human Skin Cutaneous Melanoma (SKCM): TCGA Research Network: https://www.cancer.gov/tcga -Breast dataset:

NKI: https://ccb.nki.nl/data/; Genome-Wide Gene Expression Data for 295 Samples (https://ccb.nki.nl/data/ZipFiles295Samples.zip), Clinical and other Ancillary (https://ccb.nki.nl/data/nejm table1.zip)

Normal data: https://github.com/monkgroupie/publication_code

-Kidney dataset:

Renal cancer data: GSE3538

Normal kidney data: GSE3931

LC-MS data were acquired in this study and are deposited as follows:

TMT LC-MS dataset: PXD022145 (PRIDE database of ProteomeXchange), Extended Data Figure 4d

Custom code for the DSGA analysis of breast and kidney datasets with a focus on basement membrane proteins encoding genes and processed normal breast data are available at github: https://github.com/monkgroupie/publication_code

Code of the mathematical simulation of node dilutions in the laminin network is available: http://dx.doi.org/10.17169/refubium-28413

\section{Field-specific reporting}

Please select the one below that is the best fit for your research. If you are not sure, read the appropriate sections before making your selection. $\bigotimes$ Life sciences Behavioural \& social sciences Ecological, evolutionary \& environmental sciences

For a reference copy of the document with all sections, see nature.com/documents/nr-reporting-summary-flat.pdf

\section{Life sciences study design}

All studies must disclose on these points even when the disclosure is negative.

Sample size

Sample size was calculated based on previous experience and pilot experiments

Data exclusions

No data excluded

Replication

All experiments were repeated at least 3 times in vitro and 2 times in vivo. All attempts at replication have been successful

Randomization -All knockout and wildtype litters from het $x$ het breedings were used for the groups

-Cancer cell spheres were prepared on one plate and randomly picked to be inoculated into different matrices

-Cancer cells used in the proliferation and contraction assay were plated from one setup and onto the same plate. After cells have been attached, random wells have been treated with the respective protein amounts.

-Optical tweezer experiments were performed by randomly measuring single cancer cells inside the matrix.

-Different persons performed all animal experiments and analysis. Blinded cell inoculation and histological analysis.

-Sphere formation assays have been analyzed by a second person in a blinded manner.

-Pressure myography analysis: Animal were provided with a genotype independent number and were allocated to experimental cohorts at random, ensuring blinding of the researcher and randomization of animals.

-Indentation type (IT) AFM experiments have been performed in a non-blinded manner, because IT-AFM generates objective quantitative data (Young's moduli), which is not influenced by subjective judgements of the examiner. In addition, some of the IT-AFM experiments, such as the titration experiments shown Extended Data Figure 10 cannot be performed in a blinded manner, because increasing amounts of netrin 4 is added by the examiner during the experiment. 


\section{Reporting for specific materials, systems and methods}

We require information from authors about some types of materials, experimental systems and methods used in many studies. Here, indicate whether each material, system or method listed is relevant to your study. If you are not sure if a list item applies to your research, read the appropriate section before selecting a response.

\begin{tabular}{l|l} 
Materials \& experimental syste \\
\hline $\mathrm{n} / \mathrm{a}$ & Involved in the study \\
$\square$ & $\bigotimes$ Antibodies \\
$\square$ & $\square$ Eukaryotic cell lines \\
$\square$ & $\square$ Animals and other organisms \\
$\square$ & $\square$ Clinical data
\end{tabular}

\begin{tabular}{l|l}
\multicolumn{2}{l}{ Methods } \\
\hline n/a & Involved in the study \\
\hline & $\square$ ChIP-seq \\
$\square$ & $\square$ Flow cytometry \\
$\square$ & $\square$ MRI-based neuroimaging
\end{tabular}

\section{Antibodies}

Antibodies used

-rabbit anti-netrin-4, self-made, origin: Manuel Koch lab (Cologne), rabbit immunized with the recombinant protein of netrin-4, name: KR1

-rat anti-laminin gamma 1 clone A5, RRID antibody ID: AB_1587233, Millipore, Cat.: MAB1914P, Lot.: 3046684

-goat anti-collagen IV, RRID antibody ID: AB_92262, Millipore, Cat.: AB769, Lot.: 3429095

-alphaSMA, RRID antibody ID: AB_2223021, Abcam, Cat.: ab5694, Lot.: GR3183259-39

-F4/80 clone BM8, RRID antibody ID: RRID:AB_467559, eBioscience, Cat.: 14-4801-85, Lot.: 4339486

-CD8a, Cell Signaling, Cat.: 98941 , clone D4W2Z, Lot.: 5

-Dako Envision+ System-HRP labelled Polymer Anti Rabbit, Cat. No. K4003, Lot. No. 10139088

-Dako Envision+ System-HRP labelled Polymer Anti Mouse, Cat. No. K4001, Lot. No. 10091245

-Alexa Flour 488 Donkey anti-Rabbit IgG, Cat. No. A211206, Lot. No. 1834802

-Alexa Flour 594 Donkey anti-Rat IgG, Cat. No. A21209, Lot. No. 1905801

-Alexa Flour 647 Donkey anti-Goat IgG, Cat. No. A21447, Lot. No. 1739289

polyclonal KR1 (rabbit anti-netrin-4 antibody) has been validated in Schneiders et al., JBC, 2007 using other netrin family members. Moreover, the final validation has been performed staining wildtype and netrin-4 knockout mice published in Li et al., Experimental Eye Research 2012.

polyclonal goat anti-Collagen Type IV antibody, specificity: Recognizes Human and bovine type IV collagen as demonstrated by ELISA. Less than $10 \%$ cross reactivity with collagen types I, II, III, V, and VI. May show reactivity to type IV collagen from other species. AB769 has not been tested with other extracellular matrix proteins (e.g., laminin, fibronectin).

Immunogen: Human and bovine placental collagen type IV

Species reactivity: bovine, human, mouse

References: available on RRID webpage

-Mayorca-Guiliani, A. E. et al. Decellularization and antibody staining of mouse tissues to map native extracellular matrix structures in 3D. Nat Protoc, doi:10.1038/s41596-019-0225-8 (2019).

-Localization of AQP5 during development of the mouse submandibular salivary gland.

Larsen, HS; Aure, MH; Peters, SB; Larsen, M; Messelt, EB; Kanli Galtung, H

Journal of molecular histology 42 71-81 2011

-ROCK1-directed basement membrane positioning coordinates epithelial tissue polarity.

Daley, WP; Gervais, EM; Centanni, SW; Gulfo, KM; Nelson, DA; Larsen, M

Development (Cambridge, England) 139 411-22 2012

-The histone methyltransferase Setd8 acts in concert with c-Myc and is required to maintain skin.

Driskell, I; Oda, H; Blanco, S; Nascimento, E; Humphreys, P; Frye, M

The EMBO journal 31 616-29 2012

-Type IV collagen stimulates pancreatic cancer cell proliferation, migration, and inhibits apoptosis through an autocrine loop.

Öhlund, D; Franklin, O; Lundberg, E; Lundin, C; Sund, M

BMC cancer 131542013

-In vitro modeling of the neurovascular environment by coculturing adult human brain endothelial cells with human neural stem cells.

Chou, $\mathrm{CH}$; Sinden, JD; Couraud, PO; Modo, M

PloS one 9 e106346 2014

-LIM kinase regulation of cytoskeletal dynamics is required for salivary gland branching morphogenesis.

Ray, S; Fanti, JA; Macedo, DP; Larsen, M

Molecular biology of the cell 25 2393-407 2014

-The pre- and post-somatic segments of the human type I spiral ganglion neurons--structural and functional considerations

related to cochlear implantation. 
Liu, W; Edin, F; Atturo, F; Rieger, G; Löwenheim, H; Senn, P; Blumer, M; Schrott-Fischer, A; Rask-Andersen, H; Glueckert, R Neuroscience 284 470-82 2015

-Three-dimensional spheroid cell culture of umbilical cord tissue-derived mesenchymal stromal cells leads to enhanced paracrine induction of wound healing.

Santos, JM; Camões, SP; Filipe, E; Cipriano, M; Barcia, RN; Filipe, M; Teixeira, M; Simões, S; Gaspar, M; Mosqueira, D;

Nascimento, DS; Pinto-do-Ó, P; Cruz, P; Cruz, H; Castro, M; Miranda, JP

Stem cell research \& therapy 6902015

-Macromolecular organization and fine structure of the human basilar membrane - RELEVANCE for cochlear implantation.

Liu, W; Atturo, F; Aldaya, R; Santi, P; Cureoglu, S; Obwegeser, S; Glueckert, R; Pfaller, K; Schrott-Fischer, A; Rask-Andersen, H

Cell and tissue research 360 245-62 2015

monoclonal rat anti-Laminin gamma 1 antibody, clone A5

Immunogen: Basement membrane protein preparation from mouse EHS tumor

Species reactivity: human, mouse

References:

-Mayorca-Guiliani, A. E. et al. Decellularization and antibody staining of mouse tissues to map native extracellular matrix structures in 3D. Nat Protoc, doi:10.1038/s41596-019-0225-8 (2019).

-Type IV collagen stimulates pancreatic cancer cell proliferation, migration, and inhibits apoptosis through an autocrine loop.

Öhlund, D; Franklin, O; Lundberg, E; Lundin, C; Sund, M

BMC cancer 131542013

-Regulation of PDGFC signalling and extracellular matrix composition by FREM1 in mice.

Wiradjaja, F; Cottle, DL; Jones, L; Smyth, I

Disease models \& mechanisms 6 1426-33 2013

polyclonal rabbit anti-alphaSMA

Immunogen: Synthetic peptide corresponding to Human alpha smooth muscle Actin ( $\mathrm{N}$ terminal)

Species reactivity: Mouse, Rat, Chicken, Guinea pig, Cow, Dog, Human, Pig

References: available on RRID webpage

-Maniati E et al. Mouse Ovarian Cancer Models Recapitulate the Human Tumor Microenvironment and Patient Response to Treatment. Cell Rep 30:525-540.e7 (2020).

-Hamard L et al. Targeting connexin37 alters angiogenesis and arteriovenous differentiation in the developing mouse retina. FASEB J 34:8234-8249 (2020).

-Gao C et al. MALAT1 Protected the Angiogenesis Function of Human Brain Microvascular Endothelial Cells (HBMECS) Under Oxygen Glucose Deprivation/re-oxygenation (OGD/R) Challenge by Interacting with miR-205-5p/VEGFA Pathway. Neuroscience 435:135-145 (2020).

-Kim OH et al. High-phytate/low-calcium diet is a risk factor for crystal nephropathies, renal phosphate wasting, and bone loss. Elife 9:N/A (2020).

-Tsukui T et al. Collagen-producing lung cell atlas identifies multiple subsets with distinct localization and relevance to fibrosis. Nat Commun 11:1920 (2020).

-de Oliveira $V$ et al. Uterine Gaq/11 signaling, in a progesterone-dependent manner, critically regulates the acquisition of uterine receptivity in the female mouse. FASEB J 33:9374-9387 (2019).

-Shamskhou EA et al. Hydrogel-based delivery of I-10 improves treatment of bleomycin-induced lung fibrosis in mice. Biomaterials 203:52-62 (2019).

-Du Y et al. PTEN improve renal fibrosis in vitro and in vivo through inhibiting FAK/AKT signaling pathway. J Cell Biochem 120:17887-17897 (2019).

-Yang X et al. Baicalein retards proliferation and collagen deposition by activating p38MAPK-JNK via microRNA-29. J Cell Biochem 120:15625-15634 (2019).

-Kenyon $\mathrm{E}$ et al. Ductal tree ablation by local delivery of ethanol prevents tumor formation in an aggressive mouse model of breast cancer. Breast Cancer Res 21:129 (2019).

-Qiao $L$ et al. microRNA-21-5p dysregulation in exosomes derived from heart failure patients impairs regenerative potential. $J$ Clin Invest 129:2237-2250 (2019).

-Sharick JT et al. Cellular Metabolic Heterogeneity In Vivo Is Recapitulated in Tumor Organoids. Neoplasia 21:615-626 (2019).

-Offer $S$ et al. Extracellular lipid loading augments hypoxic paracrine signaling and promotes glioma angiogenesis and macrophage infiltration. J Exp Clin Cancer Res 38:241 (2019).

-Aravani D et al. HHIPL1, a Gene at the 14q32 Coronary Artery Disease Locus, Positively Regulates Hedgehog Signaling and Promotes Atherosclerosis. Circulation 140:500-513 (2019).

monoclonal rat anti-F4/80, Clone BM8

Immunogen: F4/80 antigen

Species reactivity: Mouse

References: available on RRID webpage

-Cippà PE,Liu J,Sun B,Kumar S,Naesens M,McMahon AP. A late B lymphocyte action in dysfunctional tissue repair following

kidney injury and transplantation. Nature communications

-Singh K,Camera E,Krug L,Basu A,Pandey RK,Munir S, Wlaschek M,Kochanek S,Schorpp-Kistner M,Picardo M,Angel P,Niemann

C,Maity P,Scharffetter-Kochanek K. JunB defines functional and structural integrity of the epidermo-pilosebaceous unit in the skin. Nature communications

-Bang S,Xie YK,Zhang ZJ,Wang Z,Xu ZZ,Ji RR. GPR37 regulates macrophage phagocytosis and resolution of inflammatory pain. The Journal of clinical investigation

-Pincha N,Hajam EY,Badarinath K, Batta SPR,Masudi T,Dey R,Andreasen P,Kawakami T,Samuel R, George R,Danda D,Jacob PM, Jamora C. PAl1 mediates fibroblast-mast cell interactions in skin fibrosis. The Journal of clinical investigation -Qiao X, Rao P,Zhang Y, Liu L, Pang M, Wang H,Hu M, Tian X,Zhang J,Zhao Y, Wang XM, Wang C, Yu H, Guo F, Cao Q, Wang Y, Wang YM,Zhang GY,Lee VW,Alexander SI,Zheng G, Harris DCH. Redirecting TGF- $\beta$ Signaling through the $\beta$-Catenin/Foxo Complex Prevents Kidney Fibrosis. Journal of the American Society of Nephrology : JASN

-Probst P, Kopp J,Oxenius A, Colombo MP, Ritz D, Fugmann T,Neri D. Sarcoma Eradication by Doxorubicin and Targeted TNF Relies upon CD8+ T-cell Recognition of a Retroviral Antigen. Cancer research 
-Park DY,Lee J,Kim J, Kim K,Hong S, Han S, Kubota Y,Augustin HG, Ding L, Kim JW, Kim H,He Y,Adams RH, Koh GY. Plastic roles of pericytes in the blood-retinal barrier. Nature communications

monoclonal rabbit anti-CD8a, clone D4W2Z

Immunogen: Monoclonal antibody is produced by immunizing animals with a synthetic peptide corresponding to residues surrounding Asp42 of mouse CD8 a protein

Species reactivity: Mouse

References: available via the cell signalling powered by CiteAb

-Kevin Van der Jeught, et. al. 2020. ST2 as checkpoint target for colorectal cancer immunotherapy. JCI Insight

-Ugur Eskiocak, et. al. 2020. Differentiated agonistic antibody targeting CD137 eradicates large tumors without hepatotoxicity.

$\mathrm{JCl}$ Insight

-Huocong Huang, et. al. 2019. Targeting TGFßR2-mutant tumors exposes vulnerabilities to stromal TGF $\beta$ blockade in pancreatic cancer. EMBO Mol Med

-Haig Aghajanian, et. al. 2019. Targeting cardiac fibrosis with engineered T cells. Nature

-Naiara Martínez-Vélez, et. al. 2019. The oncolytic virus Delta-24-RGD elicits an antitumor effect in pediatric glioma and DIPG

mouse models. Nat Commun

\section{Eukaryotic cell lines}

Policy information about cell lines

Cell line source(s)

-HEK293 (Manuel Koch lab, Cologne, Germany; originally from Thermo Fisher Scientific: 293 EBNA Cells)

-HCmel12 (Thomas Tüting, Magedburg, Germany): Generated by the Tüting lab: Bald, T. et al. Ultraviolet-radiation-induced inflammation promotes angiotropism and metastasis in melanoma. Nature 507, 109-113, doi:10.1038/nature13111 (2014)

-E0771 (Robin Anderson, University of Melbourne, Australia): Available via ATCC; Cat.: ATCC ${ }^{\circledast}$ CRL-3461 ${ }^{\text {TM}}$; generated by Robin Anderson: Johnstone, C. N. et al. Functional and molecular characterisation of EO771.LMB tumours, a new C57BL/6-mousederived model of spontaneously metastatic mammary cancer. Dis Model Mech 8, 237-251, doi:10.1242/dmm.017830 (2015) -4T1 (Karmanos Cancer Institute, Detroit, US): Available via ATCC; Cat.: ATCC ${ }^{\circledR}$ CRL-2539 ${ }^{\text {TM}}$; generated by Fred Miller:

Aslakson, C. J. \& Miller, F. R. Selective events in the metastatic process defined by analysis of the sequential dissemination of subpopulations of a mouse mammary tumor. Cancer Res 52, 1399-1405 (1992).

-KPC (Jennifer Morton, Beatson Institute, Glasgow, UK): Generated by Jennifer Morton: Morton, J. P. et al. Dasatinib inhibits the development of metastases in a mouse model of pancreatic ductal adenocarcinoma. Gastroenterology 139, 292-303, doi:10.1053/j.gastro.2010.03.034 (2010).

-MDA-MB-231 parental (Memorial Sloan Kettering Cancer Center, New York, US): Generated by the Massagué lab: Minn, A. J. et al. Genes that mediate breast cancer metastasis to lung. Nature 436, 518-524, doi:10.1038/nature03799 (2005)

-mCAF1 and mCAF2 (Erik Sahai, The Francis Crick Institute, London, UK): Generated by the Sahai lab: Calvo, F. et al.

Mechanotransduction and YAP-dependent matrix remodelling is required for the generation and maintenance of cancerassociated fibroblasts. Nat Cell Biol 15, 637-646, doi:10.1038/ncb2756 (2013).

Authentication

None of the cell lines used were authenticated

Mycoplasma contamination

All cell lines have been tested for Mycoplasm on a regular basis and were negative

Commonly misidentified lines

(See ICLAC register)

HEK293 are commonly misidentified cells and for our study this does not play a role as we used this cell line to produce recombinant proteins and therefore the cell line is not critical and does not affect experiments. All other cell lines used in our study are not registered in ICLAC.

\section{Animals and other organisms}

Policy information about studies involving animals; ARRIVE guidelines recommended for reporting animal research

Laboratory animals

C57BL/6, all genders at the age of 8-12 weeks at the start of the experiment, except for the pressure myography where the mice have been 5 months old

Wild animals

no wild animals have been used in this study

Field-collected samples

no field-collected samples are used in this study

Ethics oversight

Animal experiments were approved by the Animal Experimentation Council of the Ministry of Environment and Food of Denmark and controlled and guided by Danish Inspectorate for Animal Experimentation (permission number 2017-15-0201-01265). 